\title{
The Magnetospheric Multiscale Magnetometers
}

\author{
C.T. Russell • B.J. Anderson · W. Baumjohann · K.R. Bromund • D. Dearborn • \\ D. Fischer · G. Le · H.K. Leinweber · D. Leneman · W. Magnes · J.D. Means • \\ M.B. Moldwin · R. Nakamura · D. Pierce · F. Plaschke · K.M. Rowe · J.A. Slavin • \\ R.J. Strangeway · R. Torbert · C. Hagen · I. Jernej • A. Valavanoglou • I. Richter
}

Received: 20 December 2013 / Accepted: 12 June 2014 / Published online: 20 August 2014

(C) The Author(s) 2014. This article is published with open access at Springerlink.com

\begin{abstract}
The success of the Magnetospheric Multiscale mission depends on the accurate measurement of the magnetic field on all four spacecraft. To ensure this success, two independently designed and built fluxgate magnetometers were developed, avoiding single-point failures. The magnetometers were dubbed the digital fluxgate (DFG), which uses an ASIC implementation and was supplied by the Space Research Institute of the Austrian Academy of Sciences and the analogue magnetometer (AFG) with a more traditional circuit board design supplied by the University of California, Los Angeles. A stringent magnetic cleanli-
\end{abstract}

C.T. Russell (凶) · D. Dearborn · H.K. Leinweber · D. Leneman · J.D. Means · K.M. Rowe ·

R.J. Strangeway

University of California, Los Angeles, 603 Charles Young Drive, Los Angeles, CA 90095-1567, USA

e-mail: ctrussell@igpp.ucla.edu

B.J. Anderson

Applied Physics Laboratory, The John Hopkins University, 11100 Johns Hopkins Road, Laurel, MD

20723, USA

W. Baumjohann · D. Fischer · W. Magnes · R. Nakamura · F. Plaschke · C. Hagen · I. Jernej ·

A. Valavanoglou

Space Research Institute, Austrian Academy of Sciences, Schmiedlstr. 6, 8042 Graz, Austria

K.R. Bromund · G. Le

Goddard Space Flight Center, 8800 Greenbelt Road, Greenbelt, MD 20771, USA

M.B. Moldwin · J.A. Slavin

University of Michigan, 1032 Green Street, Ann Arbor, MI 48109, USA

D. Pierce

Jet Propulsion Laboratory, 4800 Oak Grove Drive, Pasadena, CA 91109, USA

R. Torbert

University of New Hampshire, 105 Main Street, Durham, NH 03824, USA

I. Richter

Institut fuer Geophysik und Extraterr. Physik, Technische Universitaet Braunschweig,

Mendelssohnstr. 3, 38106, Braunschweig, Germany 
ness program was executed under the supervision of the Johns Hopkins University's Applied Physics Laboratory. To achieve mission objectives, the calibration determined on the ground will be refined in space to ensure all eight magnetometers are precisely inter-calibrated. Near real-time data plays a key role in the transmission of high-resolution observations stored on board so rapid processing of the low-resolution data is required. This article describes these instruments, the magnetic cleanliness program, and the instrument pre-launch calibrations, the planned in-flight calibration program, and the information flow that provides the data on the rapid time scale needed for mission success.

Keywords Magnetosphere $\cdot$ Reconnection $\cdot$ Magnetometer

\section{Introduction}

The Sun and the Earth both contain magnetic dynamos, rotating heat engines in which convection of the conducting fluid does work in the form of generating a magnetic field. Further, the Sun generates a supersonically flowing plasma that bathes all the planets with this magnetized plasma. The Earth has sufficient gravity and is sufficiently far from the Sun that it retains a substantial atmosphere, sufficient to sustain life. However, in the upper atmosphere, the ultraviolet and X-ray emissions of the Sun ionize the atmosphere so that a large fraction of the magnetic envelope surrounding the Earth is filled with low-energy plasma. The interaction of the magnetized solar wind from the Sun with the Earth's magnetized plasma energizes a small portion of the charged particle population to high energies. This energization results from magnetic reconnection both on the dayside and in the magnetotail. Many of these particles become trapped in the radiation belts of the Earth where they can be further accelerated and become a hazard to the operational spacecraft on which the population of the Earth is now quite dependent. The Magnetospheric Multiscale mission as described in accompanying articles is designed to probe how the magnetized plasmas of the solar wind and of the Earth's magnetosphere interact, and the resultant energy is stored for later release.

Since the solar wind flows past the Earth's magnetosphere much more rapidly than the speed of the fastest low-frequency wave, the fast magnetosonic wave, the interaction is supersonic, and a bow shock forms. Much free energy is given to the plasma when it passes through the shock, leading to wave phenomena and unsteadiness in the flow downstream from the shock. Most importantly, it is this shocked flow that interacts with the Earth's magnetosphere. Thus simply measuring the incoming solar wind properties prior to its passage through the shock is not sufficient to understand what conditions prevail at the magnetopause, the interface between the shocked solar wind flow, and the Earth's magnetic field. It is possible that the velocity gradient at the magnetopause and the shear in the magnetic field from the sheath to the magnetosphere might act to transfer momentum across the boundary energizing the magnetospheric plasma. While this possibility should be quantitatively studied, a different mechanism seems to be dominant at the Earth, the process called magnetic reconnection.

The Sun can be observed telescopically while the Earth's magnetosphere cannot, thus it was solar astronomy that led to the first hypotheses about magnetic reconnection. It was observed that the Sun's magnetic configuration could change very rapidly, producing fast flows that tapped the magnetic energy released in the configuration change. Since magnetic fields in a plasma store energy that can be released into the plasma, this was at some level understandable, but it was difficult to understand the rapidity with which it occurred. While most agreed that annihilation of magnetic energy was heating the plasma, and that oppositely directed magnetic fields could lead to annihilation, few understood how to achieve this 
rapid conversion of energy, in part because of their use of a two-dimensional model. Early theoretical work on this problem was done by Sweet (1958), Parker (1963), and Petschek (1964).

Some researchers, most notably R.G. Giovanelli and his post-doc J.W. Dungey, realized that the speed of a process also depended on the distance a wave had to travel. By limiting the distances involved, they could speed the process. Thus they concentrated their attention on magnetic neutral points. This approach was not, however, the majority view, and initially most attention was paid to the less efficient two-dimensional models that depended on diffusive time scales.

It soon became obvious that the Earth behaved in a similar way to the Sun with periodic rapid energizations, but for whatever reason, it was not initially obvious to most that the Earth was reacting to magnetic reconnection, even though it was clear that the Sun's magnetic field extended into space. In 1961, Jim Dungey realized how reconnection at the front and in the rear of the magnetosphere could explain many of the features of the polar cap and the aurora. He did this first for southward directed interplanetary magnetic fields (Dungey 1961), and later for northward-directed interplanetary magnetic fields (Dungey 1963). This model was developed right as the space age began, when the proper data to test these ideas were being obtained, but initially the community ignored the ideas of Dungey, and more than a decade passed before it was respectable to refer to the mechanism of reconnection as it might apply to the Earth's magnetosphere. The history of the developing understanding of reconnection is discussed in more detail in the accompanying paper on the FIELDS instrument suite by Torbert et al. (2015).

\section{Scientific Objectives of the Magnetic Field Investigation}

Early measurements of the magnetosphere were principally concentrated in one of three orbits: low-altitude polar, geostationary, and high-altitude elliptical, near the equator. Such missions included the early Interplanetary Monitoring Platforms (IMP) spacecraft, the Advanced Technology Satellites (ATS) series, and the Orbiting Geophysical Observatories (OGO) spacecraft. The odd numbered spacecraft of this latter series were highly elliptical and were launched in 1964, 1966, and 1968. The last highly elliptical mission, OGO-5, worked very well and allowed the detection of the response of the magnetopause to southward fields, which was referred to as erosion, and the reconfiguration of the magnetotail at times of substorms. These observations in turn led to a model of the substorm in which the southward turning of the magnetic field transferred magnetic flux to the geomagnetic tail and then reconnection in the geomagnetic tail led to the return of that flux to the dayside (McPherron et al. 1973; Russell and McPherron 1973). This became known as the nearEarth neutral point model for substorms, but obtaining definitive evidence for reconnection that would convince the skeptics remained elusive, in part because the magnetospheric boundaries were in constant motion and the predicted flows at the magnetopause were orthogonal to the look direction of the detectors that were oriented in the spin plane of the spacecraft. In 1977, a mission to solve these two problems was launched, called the International Sun Earth Explorers 1 and 2. These two spacecraft co-orbited in a high-altitude elliptical orbit with an adjustable separation. This configuration allowed the motion of the boundary to be measured and the precise distances and thickness of boundaries to be measured. ISEE-1 also had a detector measuring the flow in the north-south direction. Then finally, Paschmann et al. (1979) announced that the flow predicted by reconnection theory 
was present, and reconnection became accepted by the community as the principal mechanism for energizing the Earth's magnetosphere, albeit with a strident minority opposing this mechanism well into the 21 st century.

While ISEE 1 and 2 were a breakthrough pair of spacecraft in establishing the existence of reconnection, two spacecraft are not enough to determine the currents flowing in these boundaries. The next major mission, the International Solar Terrestrial Program, was another multi-spacecraft mission, placing three spacecraft in widely separated orbits. This was good for dynamics but not good for microphysics. The Polar spacecraft was a high-altitude elliptical orbiter whose line of apsides precessed around the Earth, with single-spacecraft measurements of reconnection on the tail-lobe magnetopause near the polar cusp and in the near-Earth tail. Also its companions, Geotail and Wind, as well as some other "independent" spacecraft like AMPTE/IRM and Equator-S furthered our understanding of the reconnection process in the magnetotail and at the magnetopause, mainly because of much improved particle instrumentation. But all relied on single spacecraft observations of this highly dynamic and complex process and were thus unable to clearly separate spatial and temporal effects.

In 2000, Cluster became the first four-spacecraft mission launched into an orbit similar to that of Polar. It was originally destined to measure the polar cusp which, while largely unexplored, was not expected to support much reconnection. Somewhat unexpectedly, the Cluster mission became a major contributor to the reconnection problem [e.g. Nakamura et al. 2004]. Clearly reconnection was not just the purview of the subsolar magnetopause, but could occur anywhere anti-parallel magnetic fields would appear. However, Cluster had somewhat limited time resolution and usually larger spacecraft separation, providing insight into reconnection on only the ion scale, barely approaching the more important electron scale needed in the electron-diffusion region. Cluster whetted the appetite for a fourspacecraft mission to directly address the structure of the reconnection region. Eventually such a mission was approved by NASA, the Magnetospheric Multiscale mission (MMS), ultimately scheduled to be launched in 2015. Further comparisons of the MMS and Cluster missions are given by Torbert et al. (2015).

The science of reconnection is now understood qualitatively. Magnetic field lines are defined by electron motion. To have magnetic fields swap partners so that they become connected to different regions requires that the electrons are prevented from following their earlier path. This can only happen on the electron gyroscale. Hence the magnetic field must be measured precisely over a small region which contains the neutral or reconnection point. Not only does the calculation of the magnetic geometry and the currents require a precise magnetic field measurement at four locations, but many of the other instruments require good local magnetic measurements. The mission cannot achieve its reconnection objectives without excellent magnetic observations on all four spacecraft. The need to understand magnetic reconnection is the principal driver of the measurement objectives of the MMS magnetometers. Since the mission cannot succeed without accurate magnetic measurements on all four spacecraft, magnetometers with maximum redundancy, with different heritage, and provided by different groups, were included. The sensors are similar, while not identical, but the electronic operating principles are quite different.

These data are to be completely shared among the team. There is one processing line. The data are continually being inter-compared. There is one magnetometer team who work together with common goals. The magnetometer provided by the Space Research Institute of the Austrian Academy of Science has been called the Digital Fluxgate magnetometer (DFG), and that provided by the University of California, Los Angeles has been called the Analogue Fluxgate magnetometer (AFG). This paper describes these two instruments, their pre-flight and post-flight calibration procedures, the magnetic cleanliness program, and the 
b)

a)
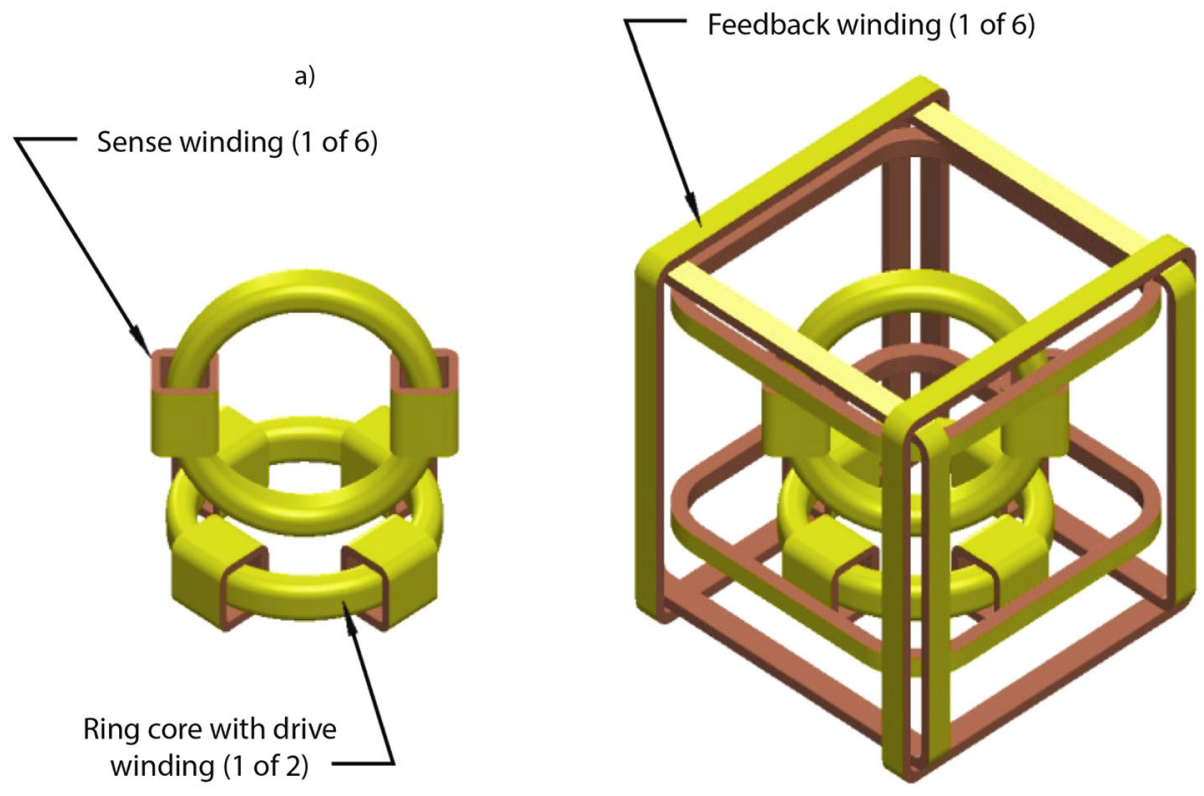

Fig. 1 (a) The magnetic ring cores, with their drive windings, the sense windings, and their relative positions and orientations. (b) The elements shown in part (a) situated within a set of feedback windings

data flow being developed to support the operations that will enable the selection of the optimum set of data to be transmitted to the ground and will provide accurate timely data for analysis.

\section{Instrument Description}

The magnetic field measurements on each spacecraft are acquired using two triaxial fluxgate magnetometers, one of each mounted on the end of two 5-m booms, each connected to an electronics unit on the main body of the spacecraft, one provided by the Space Research Institute of the Austrian Academy of Sciences, the digital fluxgate (DFG), and the other provided by the University of California, Los Angeles, the analogue fluxgate (AFG). In the following sections, we describe first the sensors that are identical except for the placement of a capacitor, and then the electronics that are based on different fluxgate magnetometer design principles.

\subsection{Sensors}

The essential components of each sensor are two magnetic ring cores, possessing wire windings to drive them into saturation, with another set of wire windings for sensing time varying magnetic flux in the cores, and a set of ambient field canceling wire windings, that enable the feedback mode of operation (see Fig. 1). Ancillary items include two printed circuit boards (PCB's), a 'pig-tail' harness, armatures and a thermistor (see Fig. 2). The boards 
b)

a)
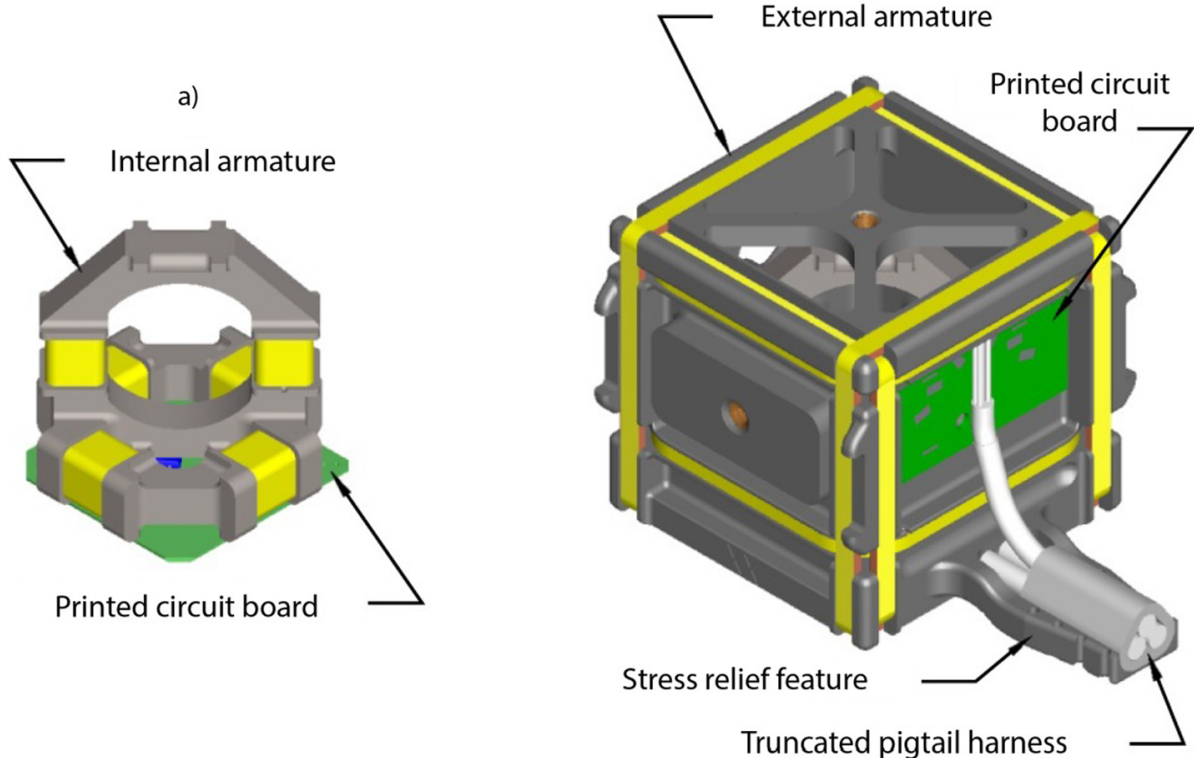

Fig. 2 (a) The internal armature, which houses the ring cores and their drive windings. The armature also defines the geometry and relative orientations of the sense windings, and provides a mounting for one of the PCB's. The thermistor (blue and partially visible) is mounted on the PCB. (b) The subassembly shown in (a) mounted in the external armature, which defines the geometry and relative orientations of the feedback windings, provides a mounting for the other PCB and the pigtail harness (shown truncated), and incorporates attachment features for the boom interface

and harness provide a conduit for electrical signal output and input to and from the windings, thermistor, and the electronics unit (see Sect. 3.2). The armatures define the sense and feedback winding geometries and the mutually orthogonal orientations of the windings and the cores. In addition, the armatures provide mounting points for the PCB's, harness and spacecraft boom. Each sensor (excluding the pigtail and its stress relief feature) has a mass of $64 \mathrm{gm}$ and measures $42.4 \times 44.3 \times 48.7 \mathrm{~mm}^{3}$. The pigtail harness (including the stress relief feature, connector, and back-shell) has a mass of $88 \mathrm{gm}$, and is $725 \mathrm{~mm}$ in length. The shielded cable section of the harness is $8.5 \mathrm{~mm}$ in diameter.

When the sensor is operating in feedback mode, the electronics unit cyclically measures the sense winding signal (which scales with the ambient magnetic field permeating the sensor), drives current in the feedback coils calculated to cancel the measured field, and then rechecks the resulting sense winding signal, searching for a minimum. The field strength reported to the ground is the feedback coil-generated field strength required to cancel the ambient field at the sensor. Figure 3 is a photograph of the completed sensor.

Before launch the functionality of the magnetometers must be periodically verified. As their dynamic range is less than the strength of the Earth's magnetic field, this field must be attenuated to run the test. A combination of wire windings, called a nulling coil assembly, is used for this purpose. The assembly is shown in Fig. 4; power supplies drive currents in the windings to generate sufficient field to bring the magnetic field local to the sensor within its dynamic range. 
Fig. 3 Photograph of the fluxgate sensor with pigtail

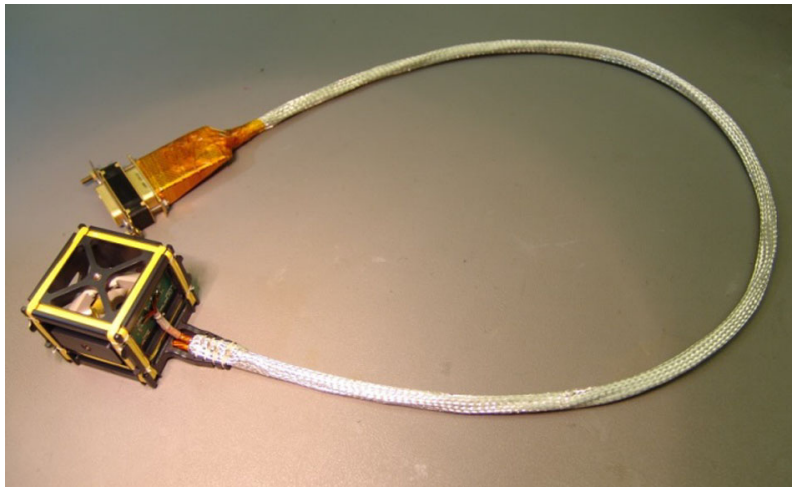

Fig. 4 The nulling coil assembly (red), shown mounted around the magnetometer, at the end of the magnetometer boom. The magnetometer boom (black) ends in a y-shaped yoke that attaches to the magnetometer with 2 brass screws, one of which is partly visible in this view. As the nulling coil is ground service equipment and must be removed before launch, it is so labeled and colored red

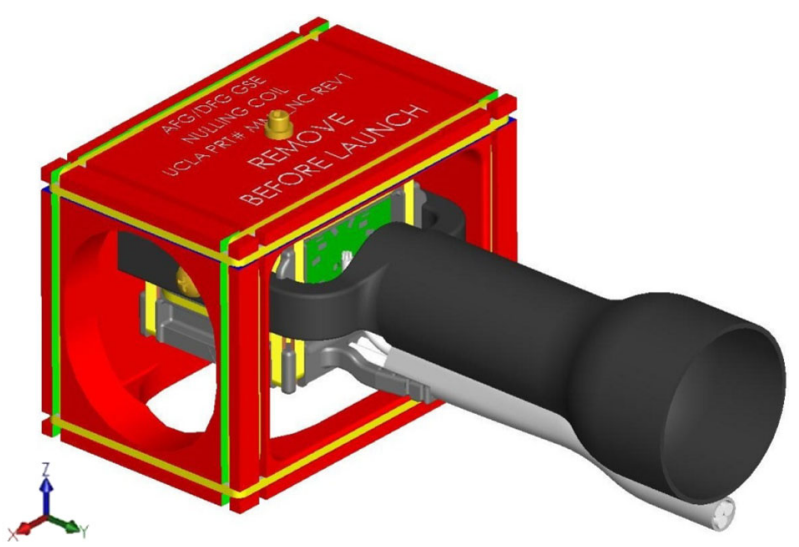

\subsection{Electronics}

An accurate measurement of the magnetic field is the sine qua non of the Magnetospheric Multiscale Mission. Without accurate low-noise magnetic field measurements, we would not be able to achieve the objectives of the mission. Simple redundancy was not enough to ensure success because two units with the same fault could doom the mission. Rather, the two magnetometers had to have different heritage and design, as well as extensive testing and quality control. The use of common sensors was not considered to be a violation of this rule because the sensors were essentially passive devices, but the electronics units had to be quite distinct in design.

\subsubsection{Digital Fluxgate Magnetometer}

The Digital Fluxgate Magnetometer (DFG) is composed of a miniaturized ASIC (Application Specific Integrated Circuit) based sensor electronics developed by the Space Research Institute (IWF) in Austria (see block diagram in Fig. 5) and a new sensor design, which was manufactured and qualified by the University of California, Los Angeles (UCLA). Both instrument requirements and main instrument parameters are listed in Table 3.

The Magnetometer Front-end ASIC (MFA) was developed in a close cooperation between the IWF magnetometer group and the Fraunhofer Institute for Integrated Circuits 


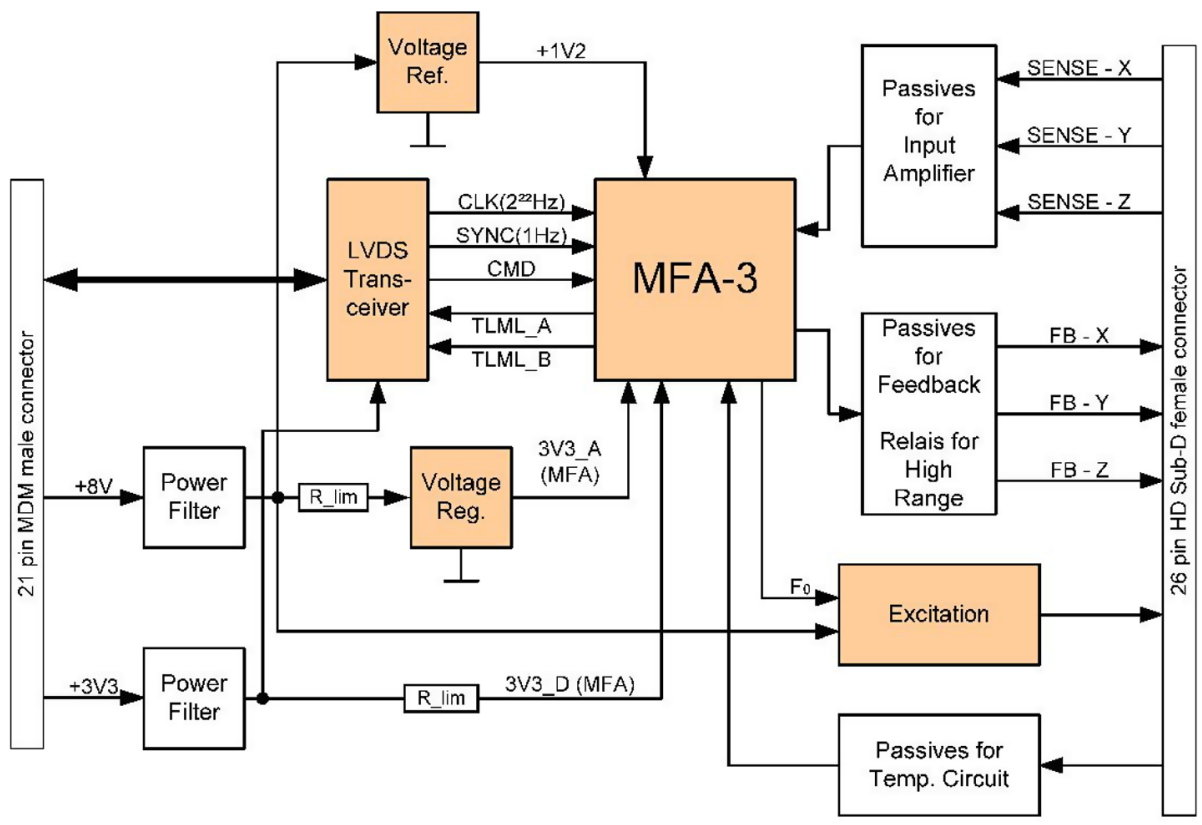

Fig. 5 Board level block diagram of the DFG sensor electronics with five active (orange blocks) and five passive circuit elements (white blocks)

under an ESA contract in the years 2004 to 2007 (Magnes et al. 2008), and is shown in Fig. 6.

All the active electronics needed for the readout of the fluxgate sensor and the digitization of the magnetic field and housekeeping data are implemented in the MFA. Active electronics outside of the MFA are reduced to driver components for the digital interface and the excitation of the fluxgate sensor, a voltage reference and a voltage regulator. The entire electronics are assembled on an $7 \times 11 \mathrm{~cm}^{2}$ printed wire board, which is attached to the AFG sensor electronics inside the Field's Central Electronics Box (CEB), as shown in Fig. 7. The DFG specifications are listed in Table 1.

The analogue part of the MFA (14,000 transistors) contains altogether four 2-2 cascaded sigma-delta modulators for high resolution analogue-to-digital conversion. Three of those modulators are connected to the fluxgate sensor and have modified first stages for an appropriate tuning of the MFA to the fluxgate sensor. The fourth modulator is connected to the output of an eight-to-one multiplexer for housekeeping measurements (e.g. temperatures of MFA and fluxgate sensor). The single-bit outputs of the cascaded modulators are processed by a digital tuning logic for generating a fourth-order noise shaped and digitized output signal with 6-bit data width at a sampling rate of $8,192 \mathrm{~Hz}$, when the chip is clocked at $2^{22} \mathrm{~Hz}$ (about 4.2 MHz).

The digital part (25,000 digital gates) includes primary (128 Hz output) and secondary decimation filters $(2,4,8$, to $64 \mathrm{~Hz}$ output) as well as a serial synchronous interface. The chip area (0.35 $\mu \mathrm{m}$ CMOS process from "austriamicrosystems") is about $22 \mathrm{~mm}^{2}$ (Fig. 2) and the total power consumption of just the MFA is $60 \mathrm{~mW}$ which does not include the excitation power for the fluxgate sensor. The MFA operates within its specifications up to $170 \mathrm{krad}$ of total ionizing dose and shows full functionality up to more than $300 \mathrm{krad}$. 


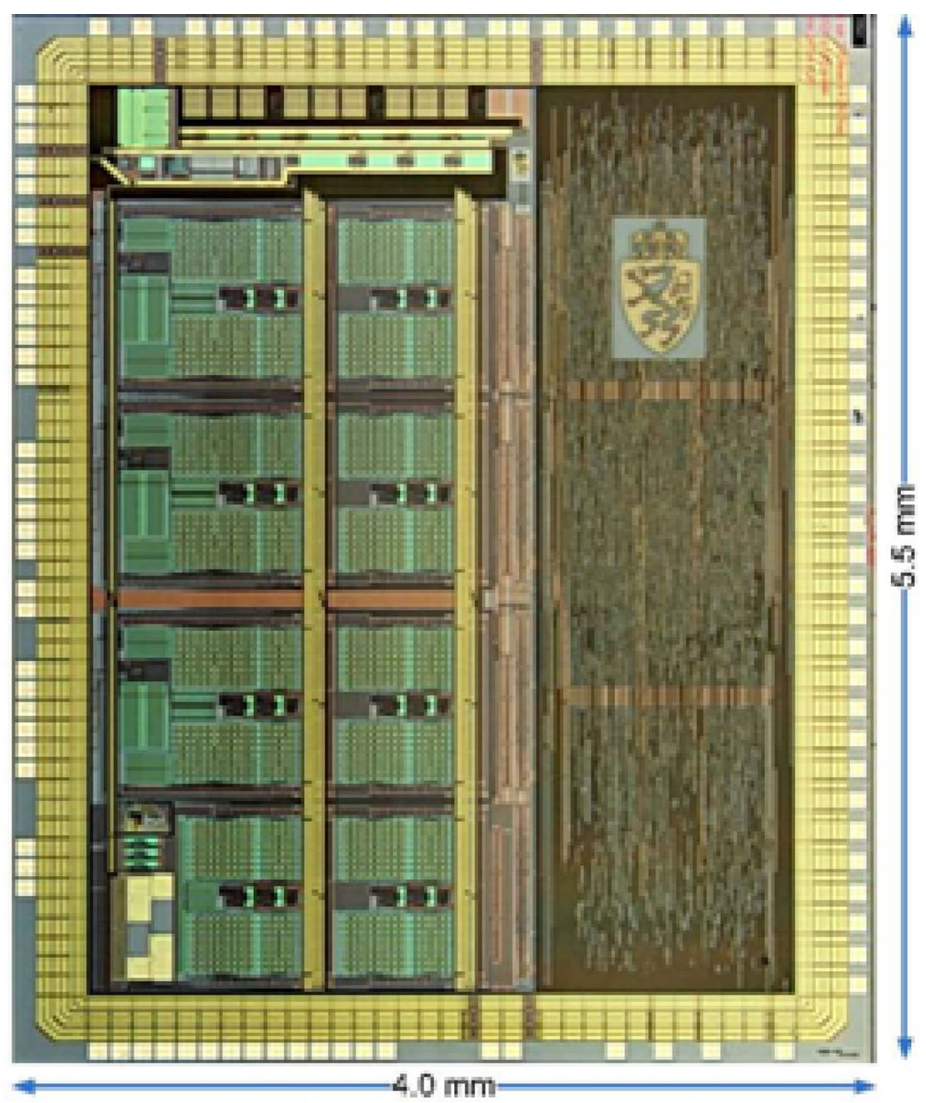

Fig. 6 Photograph of the magnetometer front-end ASIC with an enlargement of the Styrian panther which in reality has a size of $0.1 \times 0.2 \mathrm{~mm}^{2}$. The left side of the ASIC contains four sigma-delta loops for the magnetic field as well as the housekeeping measurements and the right part is composed of mainly digital circuits for data decimation, clock generation and a serial synchronous interface

For MMS, the second revision of the MFA was space qualified according to NASA rules which, e.g., includes space-qualified packaging of the dies, screening of all MFAs in the military temperature range between $-55^{\circ} \mathrm{C}$ and $125^{\circ} \mathrm{C}$, a 1000 hour long life testing of a reduced number of chips at $125^{\circ} \mathrm{C}$, and radiation testing of some MFAs by bombarding them with heavy ions.

\subsubsection{The AFG Electronics}

The Analogue Fluxgate Magnetometer (AFG) consists of three matched elements, the precision low mass sensor, the interconnecting boom cable, and the electronics board. For optimum operation the electronics and sensor are tuned as a system using an identical interconnecting cable. The AFG electronics is shown in Fig. 8. The AFG board is designed to fit inside the Central Electronics Box (CEB) of the FIELDS package, which provides power, timing, and commands, and collects the data. The lower right hand cutout of the PCB fits the DFG board. As well as digitizing the AFG sensor, the AFG electronics provides feed 


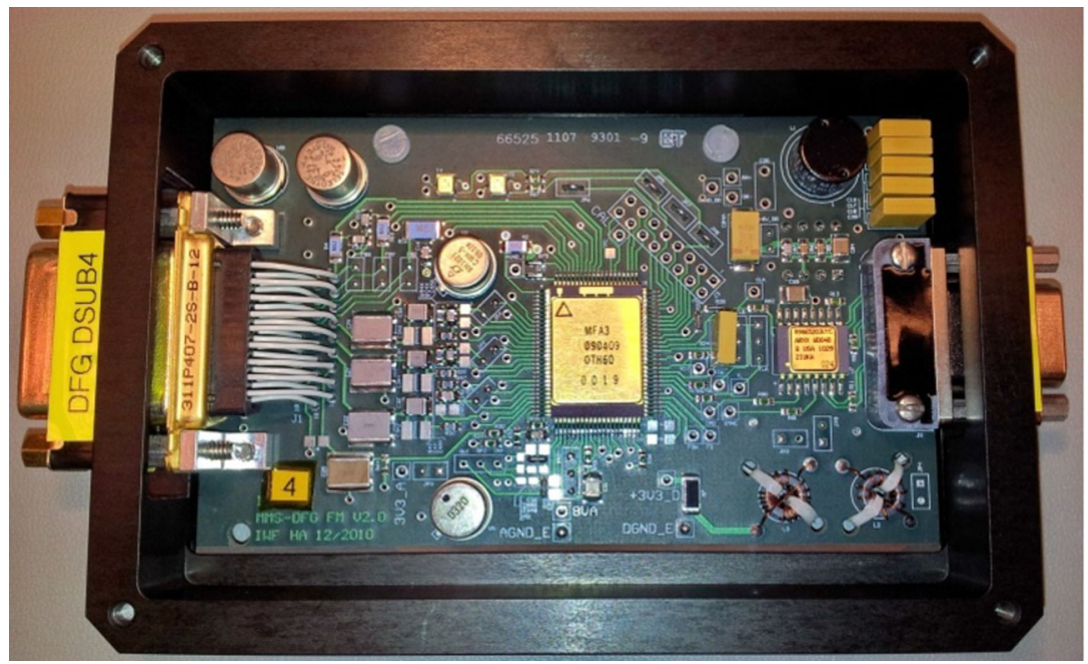

Fig. 7 Picture of the Flight Model 4 DFG electronics board mounted in the sub-unit test frame

Table 1 DFG specifications

\begin{tabular}{ll}
\hline Board size & $7 \times 11 \mathrm{~cm}^{2}$ \\
Board mass & $76 \mathrm{~g}$ \\
Supply voltages & $3.3 \mathrm{~V}$ (digital), $8 \mathrm{~V}$ (analogue) \\
Power consumption & $450 \mathrm{~mW}$ (nominal) \\
Digital resolution & $24 \mathrm{bits}$ \\
Dynamic range & $\pm 650 \mathrm{nT}$ (low range) $\pm 10,500 \mathrm{nT}$ (high range) \\
Noise density at $1 \mathrm{~Hz}$ & $<8 \mathrm{pT} / \sqrt{\mathrm{Hz}}$ (low range) $<100 \mathrm{pT} / \sqrt{\mathrm{Hz}}$ (high range) \\
Offset drift with electronics temperature & $<5 \mathrm{pT} /{ }^{\circ} \mathrm{C}\left(\right.$ low range) $<10 \mathrm{pT} /{ }^{\circ} \mathrm{C}$ (high range) \\
Offset drift with sensor temperature & $<10 \mathrm{pT} /{ }^{\circ} \mathrm{C}$ (both ranges) \\
Gain drift with electronics temperature & $<10 \mathrm{ppm} /{ }^{\circ} \mathrm{C}$ (low range) $<15 \mathrm{ppm} /{ }^{\circ} \mathrm{C}$ (high range) \\
Gain drift with sensor temperature & $<60 \mathrm{ppm} /{ }^{\circ} \mathrm{C}$ (low range) $<350 \mathrm{ppm} /{ }^{\circ} \mathrm{C}$ (high range) \\
Non-linearity & $<3 \times 10^{-5}$ (low range) $<6 \times 10^{-4}$ (high range) \\
\hline
\end{tabular}

through lines from the CEB power, timing, and commands, and data and provides mounting and connectors for the DFG.

The AFG board contains the fluxgate analogue circuit, analogue to digital converters, digital circuit, spacecraft interface circuits, and power monitoring and conditioning circuits. The AFG is based on a flight proven design. The block diagram for the electronics is given in Fig. 9.

This circuit gives a drive frequency of $16 \mathrm{kHz}$ and detects the $32 \mathrm{kHz}$ second harmonic generated by the sensors when an external magnetic field is present. The second harmonic signal is multiplexed and sampled using the LRC1604, 16-bit ADC. The AFG samples each axis 16 times in $60 \mu \mathrm{sec}$, which is filtered and output as a 24-bit sample at a rate of $128 \mathrm{~Hz}$. The three sensors are sampled sequentially. In order to improve the linearity and stability of the magnetometer, a feedback system is utilized which maintains the sensor core at near-zero field. A $16 \times$ amplifier provides the low range data. The instrument uses an AC- 
Fig. 8 AFG Electronics board
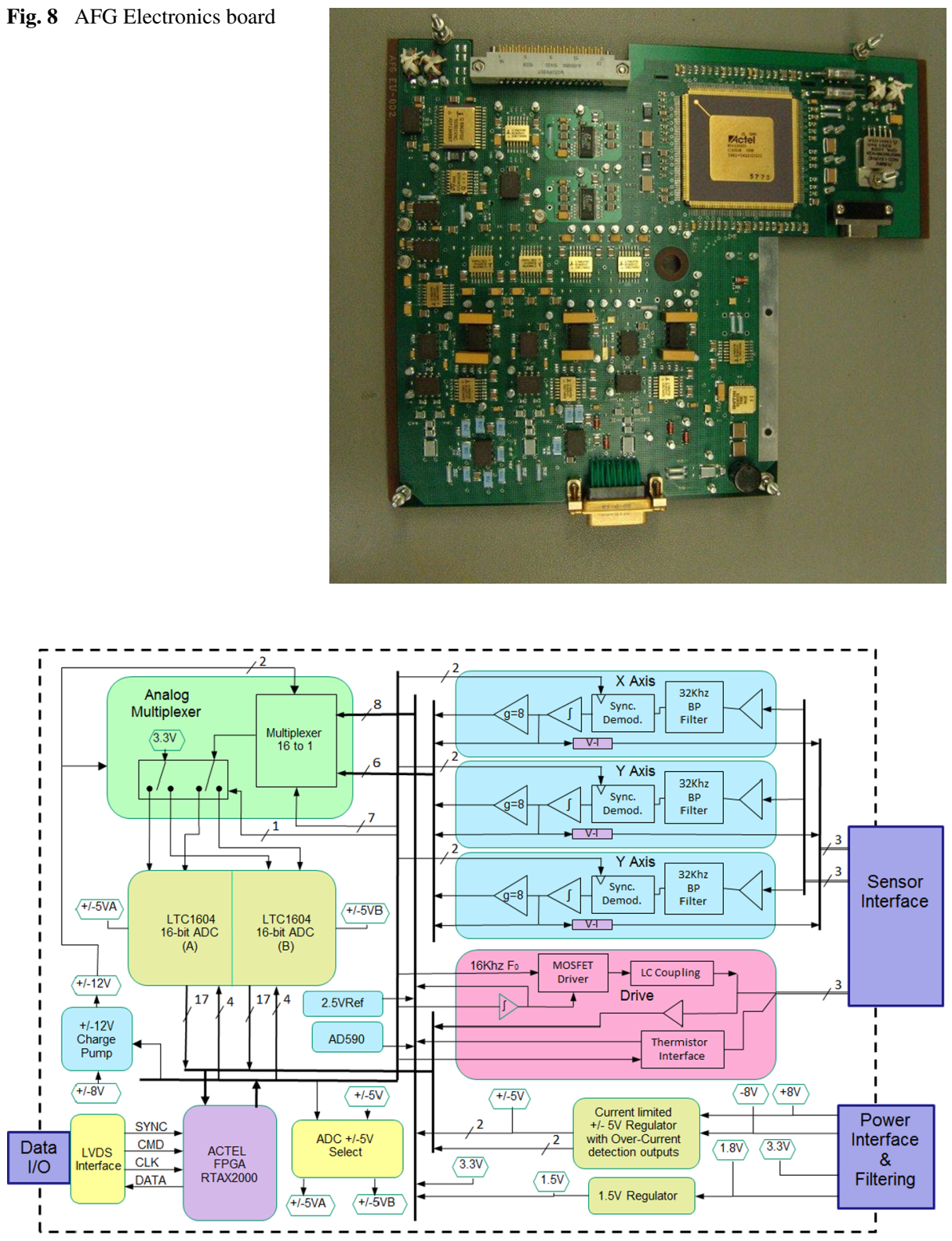

Fig. 9 AFG Block Diagram

TEL RTAX2000S/SL FPGA and redundant LT1604 A/D converters. The performance and resource requirements are shown in Table 2.

Upon power application, the AFG synchronizes with the CEB timing and then provides the output data. With the similarities in requirements for data and commands between AFG and DFG, it was decided to utilize the structure of the DFG specifications and supplement some commands to provide the control needed for the AFG. 
Table 2 Specifications of the Analogue Fluxgate Magnetometer

Fig. 10 Amplitude spectral density spectra for high range. Noise level is $\sim 8 \mathrm{pT} / \sqrt{\mathrm{Hz}}$ at $1 \mathrm{~Hz}$

Fig. 11 Amplitude spectral density for low range. Noise level is $\sim 5 \mathrm{pT} / \sqrt{\mathrm{Hz}}$ at $1 \mathrm{~Hz}$

\begin{tabular}{lll}
\hline Board Size & $23.2 \times 19.1 \mathrm{~cm}$ & \\
Board Mass & 285 grams & \\
Power Consumption & $1.2 \mathrm{~W}$ & \\
Dynamic Range & High: $\pm 8200 \mathrm{nT}$ & Low: $\pm 510 \mathrm{nT}$ \\
Noise Levels @ $1 \mathrm{~Hz}$ & High: $10 \mathrm{pT} / \sqrt{\mathrm{Hz}}$ & Low: $5 \mathrm{pT} / \sqrt{\mathrm{Hz}}$ \\
Nyquist Frequency & $64 \mathrm{~Hz}$ & \\
\hline
\end{tabular}
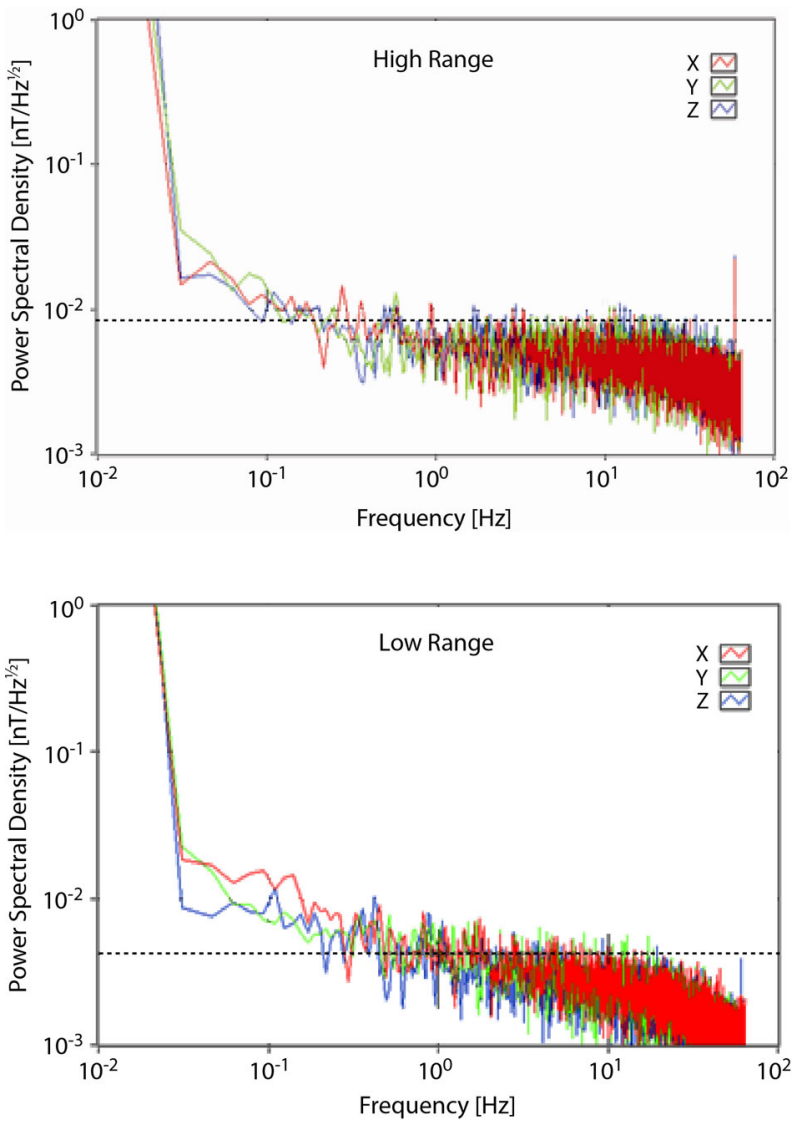

AFG transmits the three magnetic field values ( $X, Y$, and $Z$ ), status information, and the housekeeping vector. Each of the three magnetic components and the housekeeping data are 24-bit, 2's complement. The status information reflects the current status of the AFG such as the AFG ID, Data On, ADC ID, Test Mode, Range, EU ID, Overcurrent Status, Parity error, Frame error, Command error, and FPGA ID. The housekeeping data reflects the voltages and temperatures (sensor and electronics) for the AFG.

Depending on the field present, full scale ranges can be commanded to be either $\pm 8,000 \mathrm{nT}$ (high range) or $\pm 500 \mathrm{nT}$ (low range). Most of the science data will be collected at the low range, which has an increased sensitivity and lower noise, as seen in Figs. 10 and 11 . The superior stability and accuracy of the AFG magnetometer can be seen in Fig. 12 to be less than $0.1 \mathrm{nT}$ over 100 hours. 
Fig. 12100 hour stability test

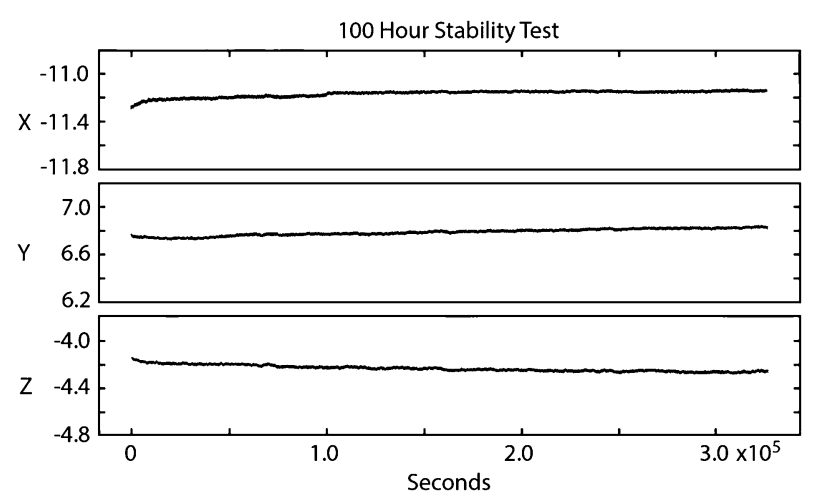

\section{Calibrations}

There are two main types of calibrations for space-borne magnetometers, namely ground calibrations and in-flight calibrations. Ground calibrations have a dual purpose. They provide a valuable functional test, and determine all twelve calibration parameters. Furthermore, the ground calibrations provide insights on temperature dependence and linearity. Most importantly, these tests occur in a controlled environment and cover the full range of expected conditions of temperature and field strength. In-flight calibrations are needed to precisely adjust calibration parameters for the encountered conditions on orbit as well as capturing changes of calibration parameters that are less stable. These calibrations are valuable as they occur close in time to the acquisition of the science data, but they are not obtained in a controlled environment. For example, the field magnitude, the field direction, and the temperature may be changing simultaneously.

A linear magnetometer can be calibrated with the twelve calibration parameters defined below. The parameters consist of three gains, three offsets and six angles (see Fig. 13).

$$
\left(\begin{array}{c}
B_{S_{1}} \\
B_{S_{2}} \\
B_{S_{3}}
\end{array}\right)=\left(\begin{array}{lll}
G_{1} \sin \theta_{1} \cos \varphi_{1} & G_{1} \sin \theta_{1} \sin \varphi_{1} & G_{1} \cos \theta_{1} \\
G_{2} \sin \theta_{2} \cos \varphi_{2} & G_{2} \sin \theta_{2} \sin \varphi_{2} & G_{2} \cos \theta_{2} \\
G_{3} \sin \theta_{3} \cos \varphi_{3} & G_{3} \sin \theta_{3} \sin \varphi_{3} & G_{3} \cos \theta_{3}
\end{array}\right) \cdot\left(\begin{array}{c}
B_{x} \\
B_{y} \\
B_{z}
\end{array}\right)+\left(\begin{array}{c}
O_{1} \\
O_{2} \\
O_{3}
\end{array}\right)
$$

$B_{S_{1}}, B_{S_{2}}, B_{S_{3}}$ non-orthogonal field components as measured by the magnetometer sensors $B_{x}, B_{y}, B_{z}$ orthogonalized field components

$G_{1}, G_{2}, G_{3}$ gain corrections of each of the sensors

$\theta_{1}, \theta_{2}, \theta_{3} \quad$ elevation angles of each of the sensors

$\varphi_{1}, \varphi_{2}, \varphi_{3} \quad$ azimuthal angles of each of the sensors

$\mathrm{O}_{1}, \mathrm{O}_{2}, \mathrm{O}_{3}$ offsets of each of the sensors

The above matrix is the inverse of a calibration matrix. The six angles describe the orientation of the sensor axes with respect to the sensor mechanical axes. The angles provide information on orthogonality as well as absolute orientation with respect to the mechanical axes.

\subsection{Ground Calibrations}

Ground calibrations are generally performed at magnetic testing facilities. There are two major kinds of such testing facilities that can be used for magnetometer calibration. The 


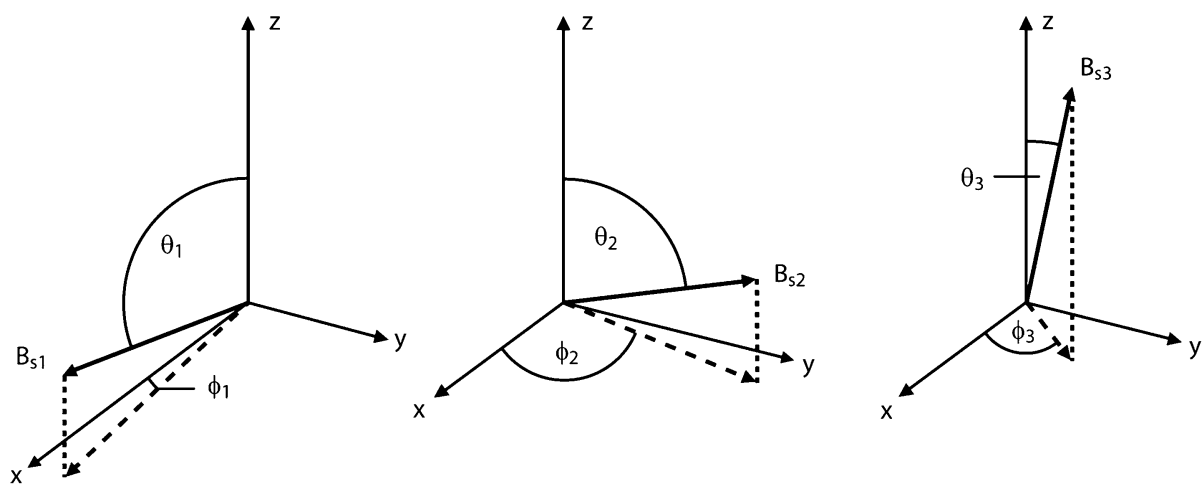

Fig. 13 Definition of six angles for magnetometer calibration. Here and in the analyses that follow, the $z$-axis is defined as the spin axis. On the spacecraft, different definitions of the coordinate systems are used

first kind is a "mu-metal" chamber that shields its inside against environmental magnetic fields. Mu-metal chambers have high attenuation factors so that changes of the external field (Earth field) have negligibly small influence on the magnetic measurements inside the chamber. The second type is a magnetically open facility that is typically located at a remote area that is magnetically quiet. The varying Earth's field must be compensated with a coil system.

\subsubsection{Facilities Used}

All DFG-magnetometers and two AFG-magnetometers were calibrated at the magnetically open test facility of TU-Braunschweig (often referenced as "Magnetsrode"). It is located inside a forest near the town of Braunschweig. The facility is equipped with a Braunbek coil system and a ground based magnetometer to compensate the varying magnetic field at the surface of Earth. Additionally, the facility is equipped with a thermal enclosure for magnetometer sensors that allows the measurement of temperature drifts of the calibration parameters. The design of a Braunbek coil system allows for a large volume of homogeneous magnetic field within a relatively small coil system (e.g. Caprari 1995).

The coil system at "Magnetsrode" (see Fig. 14) has a linear area of $20 \times 20 \times 20 \mathrm{~cm}^{3}$ ( $<1 \mathrm{nT}$ variations). Artificial magnetic fields in any direction can be generated within the homogenous field volume. The Earth field compensation is controlled by a 3-axis fluxgate magnetometer that is located 50 meters away from the coil system, inside a bunker (Glassmeier et al. 2007). The facility can be used to measure precise scale factor, offset, linearity and orthogonality in the operating temperature range from $-50{ }^{\circ} \mathrm{C}$ to $30{ }^{\circ} \mathrm{C}$ as well as for sensor alignment (sensor magnetic to mechanical axes) at environmental temperature.

The mu-metal chamber at IGPP UCLA is equipped with a Helmholtz coil system that allows the compensation of the small remaining field inside the chamber as well as the application of precise reference fields (see Fig. 15). It can be used to measure precise scale factor, offset, linearity orthogonality and alignment at room temperature. The facility was used for alignment tests of the second pair of AFG magnetometers.

Tests of the DFG magnetometers were also performed at IWF-Graz. The temperature test facility at the Magnetometer Laboratory of IWF was used for complementary measurements of noise power spectral density, sensor offset and linearity as well as a proper verification of the sensor tuning in the entire survival temperature range from $-100{ }^{\circ} \mathrm{C}$ to $60{ }^{\circ} \mathrm{C}$. The fa- 
Fig. 14 Both, AFG and DFG flight sensors in an aligned configuration in the Braunbek coil system of Magnetsrode, TU Braunschweig, for comparison measurements in a statically compensated Earth's field


Fig. 15 MMS-sensor inside the mu-metal chamber at IGPP/UCLA. The turntable is used for precise alignment tests. The Helmholtz coil compensates the remaining field inside the mu-metal chamber and can be used to apply known reference fields

cility consists of a three-layer magnetic shielding set, a combined low and high-temperature controller and an external stimulus coil.

Tests of the AFG magnetometers were also performed at the Magnetometer Laboratory at the Institute of Geophysics and Planetary Physics, UCLA. In addition to the alignment tests performed within the mu-metal chamber shown in Fig. 15, tests were conducted using shield cans and thermal chambers to test the linearity, noise levels, offsets and gains for the AFG magnetometer sensor and electronics. The test facilities include a Tenney thermal chamber that is used to conduct temperature cycling tests for the flight electronics, and two triplelayer mu-metal shield cans. The shield cans also include a well-calibrated stimulus coil for gain determination. One of the shield-can sets can also be thermally controlled, to enable temperature cycling of the sensors. The other shield-can set is used at ambient temperature when the electronics unit is being thermally cycled. 

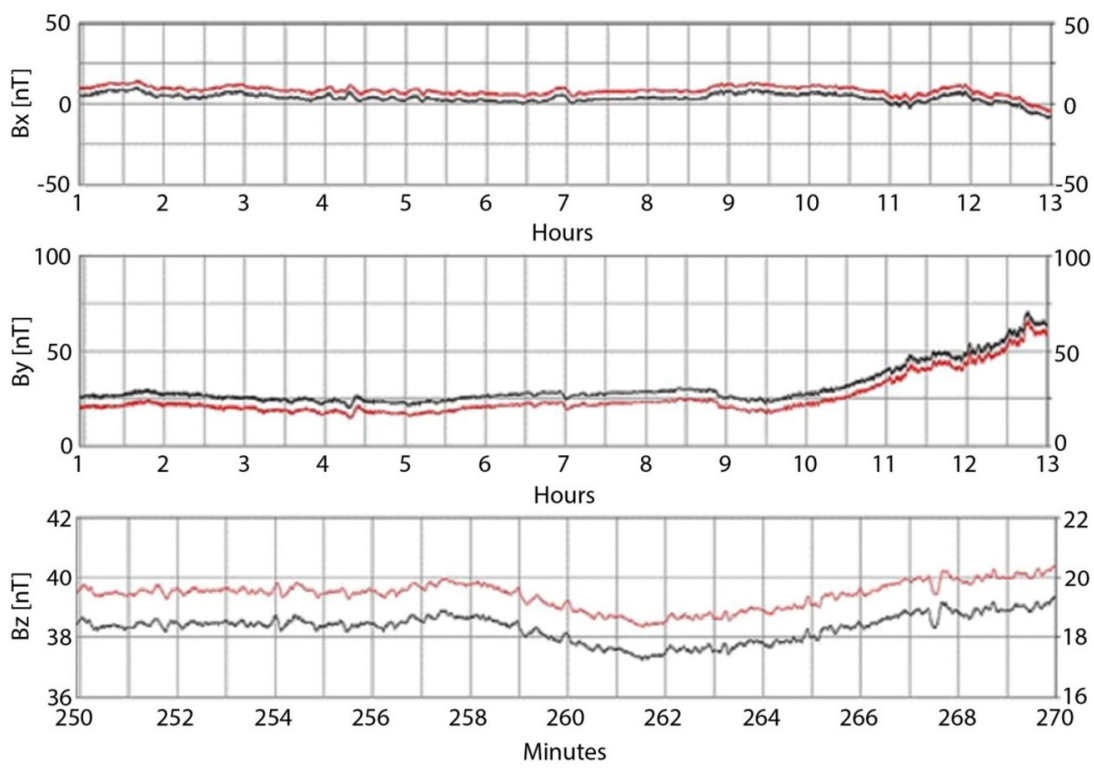

Fig. 16 Measurement of the Earth's field variation over 13 hours (20 min zoom in of $Z$ component) by manually aligned AFG and DFG sensors in the Braunbek coils system of Magnetsrode which was used for a static compensation of the Earth's field

Fig. 17 Misalignment angles of the DFG FM 2 sensor in the operating temperature range from $-50{ }^{\circ} \mathrm{C}$ to $30{ }^{\circ} \mathrm{C}$ measured at Magnetsrode. This plot was measured with the instrument in high range but the results in low range were the same while only a bit noisier

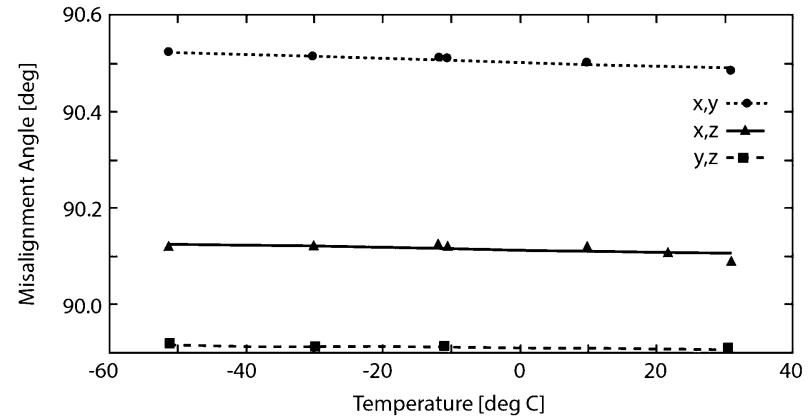

\subsubsection{DFG}

Selective calibration results gained at Magnetsrode are depicted in Fig. 16 through Fig. 18. Figure 16 shows parallel measurements of Earth field variations by the AFG and DFG magnetometers. The following two figures (Figs. 17 and 18) provide examples of measurements with the thermal enclosure. Figure 18 shows an example of sensor sensitivities versus temperature. Figure 17 shows an example of orthogonality (named misalignment in the figure) versus temperature. The noise spectral density of the DFG FM2 components in the survival temperature range from $-100{ }^{\circ} \mathrm{C}$ to $60{ }^{\circ} \mathrm{C}$ is plotted in Fig. 19. The transfer function of the DFG is shown in Fig. 20. It is dominated by the digital decimation filter of the sigma-delta conversion principle within the MFA and as such exactly the same for all DFG magnetometers. The decimation filter is furthermore a non-recursive digital filter with constant group delay. Additionally, the AFG/DFG magnetometers require a non-linearity correction for their high field range, of about $\pm 0.01 \%$ of full range. 
Fig. 18 Relative gain drift of the DFG FM 2 sensor components in the operating temperature range from $-50{ }^{\circ} \mathrm{C}$ to $30{ }^{\circ} \mathrm{C}$ measured at Magnetsrode. It was measured with the instrument commanded in low range
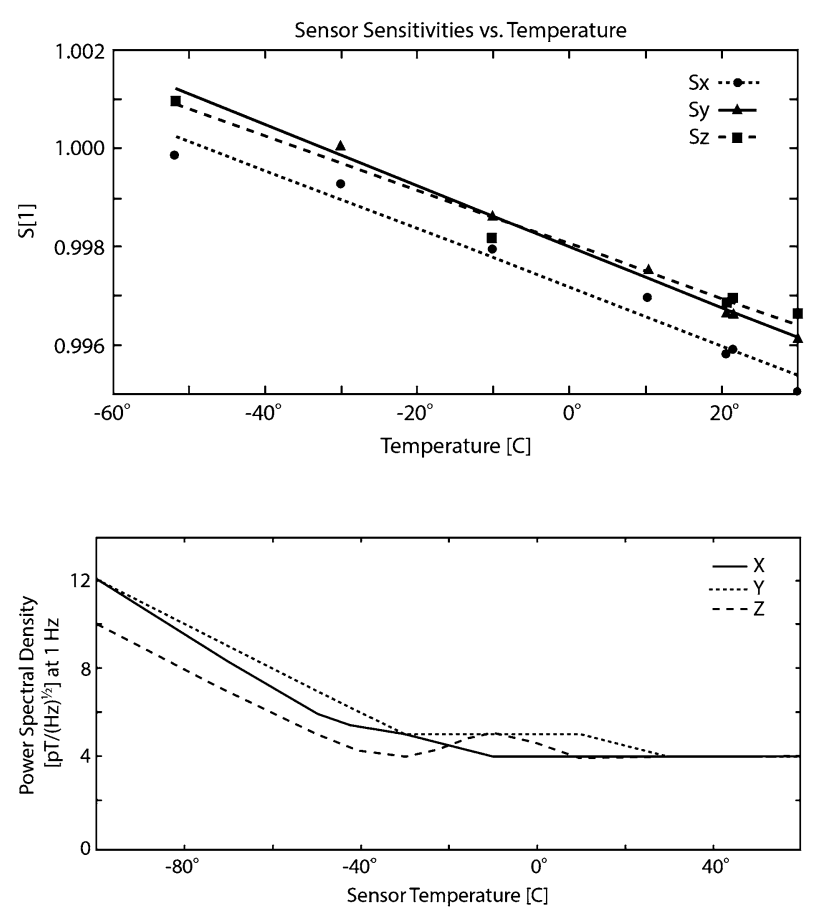

Fig. 19 Amplitude spectral density of the DFG FM2 components in the survival temperature range from $100{ }^{\circ} \mathrm{C}$ to $60{ }^{\circ} \mathrm{C}$ measured with the IWF facility. The increased noise below $-50{ }^{\circ} \mathrm{C}$ is caused by the loss of tuning precision when the sensor temperature gets outside of the operating temperature range

Fig. 20 Magnitude and delay (phase) response of the DFG magnetometers; the delay was measured relative to the time stamping done in the central data processing unit while the applied field was synchronized to a $1 \mathrm{~Hz}$ reference pulse like it is the case in the flight configuration

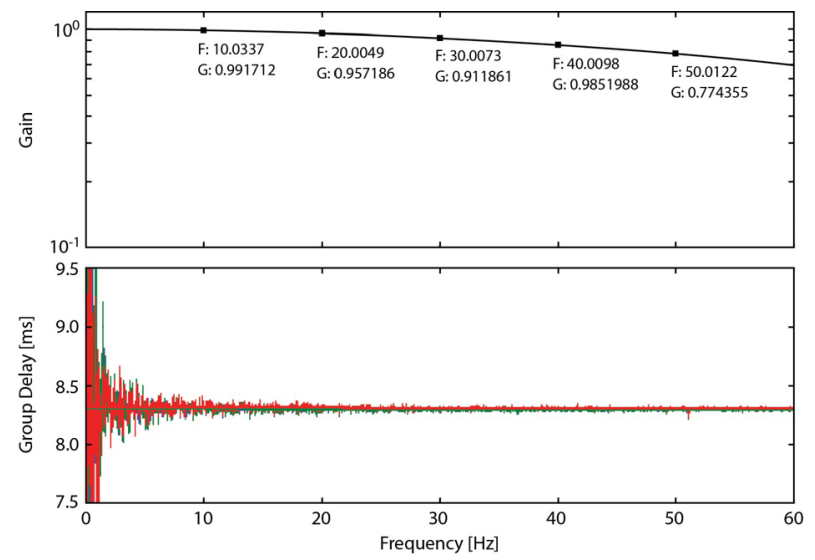

\subsubsection{AFG}

AFG FM1 and FM2 were calibrated similarly as the DFG magnetometers at the facility of TU-Braunschweig ("Magnetsrode"). AFG FM3 and FM4 were calibrated at IGPP UCLA.

Figure 21 shows an example of an alignment test at IGPP/UCLA. The test was performed by rotating the sensor with a turntable while a large perpendicular field was applied. The amplitude and the phase of the resulting sine wave provide information on the azimuthal and elevation angles of the sensor with respect to the mechanical axis. The sensor was mounted on the turntable differently three times, to measure the alignment of all sensor axes. The 
Fig. 21 Example of the rotational alignment test with the turntable inside the mu-metal chamber of AFG FM3 in low range. The solid trace in the top panel shows the response of the $\mathrm{X}$-sensor to a $490 \mathrm{nT}$ perpendicular field. The dashed trace shows the fit to the data and the bottom panel shows the residual

Fig. 22 Magnetometer linearity test results for the $z$-axis of the AFG FM3 magnetometer
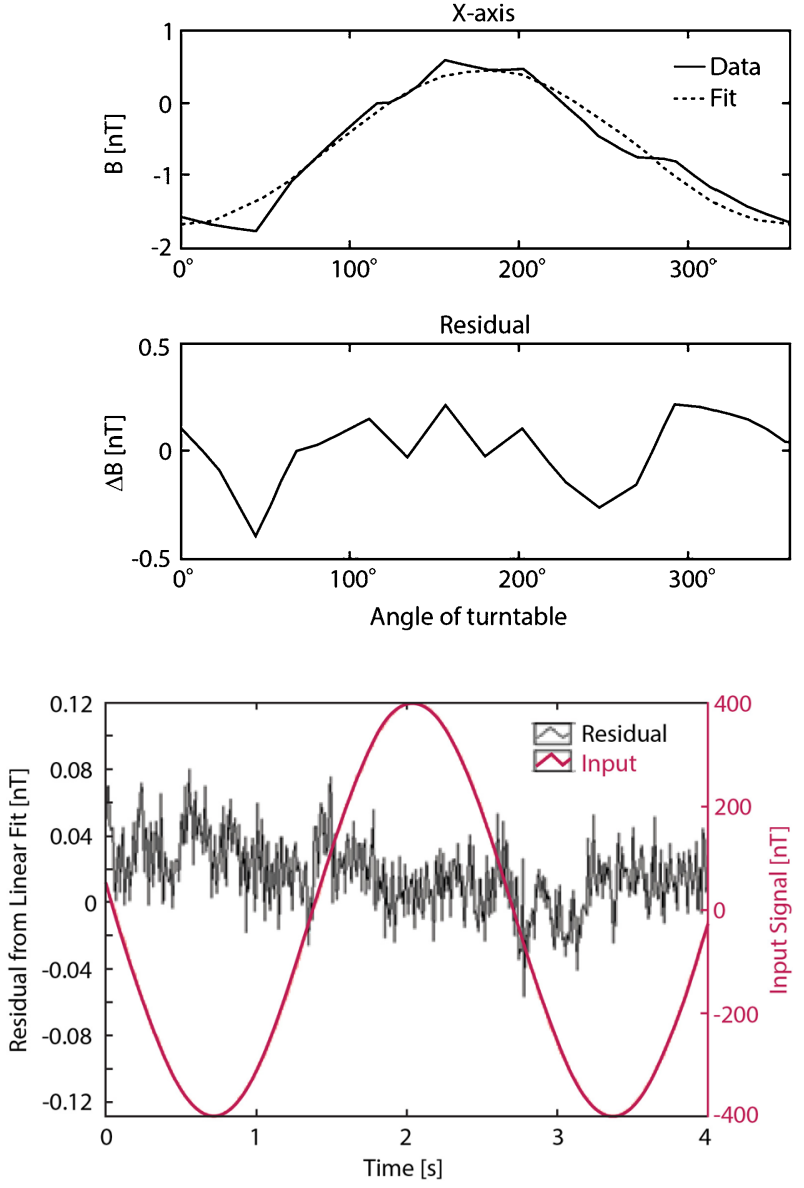

technique is robust against uncertainties of the perpendicular reference field. The data also show that the facility can be used to resolve a $\sim 1 \mathrm{nT}$ sine wave.

Examples of the calibration tests performed on the AFG magnetometer using the UCLA magnetometer laboratory test facilities discussed in Sect. 4.1.1 are shown in Figs. 22 through 26. The figures show the results of the tests performed with the FM3 flight model, with the magnetometer operating in high gain (low field) mode. This is the mode that will be used when the MMS spacecraft are in the scientific "Region of Interest." Similar tests were performed for the low gain (high field) mode, and all four AFG flight units were subject to the same tests on the electronics units. For the FM1 and FM2 sensors, the sensortemperature dependent gain and offset variation were determined with the Braunschweig test facility. For AFG FM3 and FM4 the tests were performed at UCLA using the shield cans. Earlier Figs. 10 and 11 have shown the noise levels for FM3 for the high field and low field ranges respectively. These noise levels are similar to those seen on the other units.

Figure 22 shows the results of the linearity test for AFG FM3. The magnetometer was operating in the low field mode, and $400 \mathrm{nT}$ amplitude $0.05 \mathrm{~Hz}$ sine wave was applied. The non-linearity is less than $0.1 \mathrm{nT}$ peak to peak.

Figure 23 shows the offset change with temperature for the AFG FM3 electronics unit. The variation with temperature is very linear, varying by about $2 \mathrm{nT}$ over the entire operating 
Fig. 23 AFG FM3 offset dependence on electronics temperature

Fig. 24 AFG FM3 gain dependence on electronics temperature, relative to the gain at $20^{\circ} \mathrm{C}$
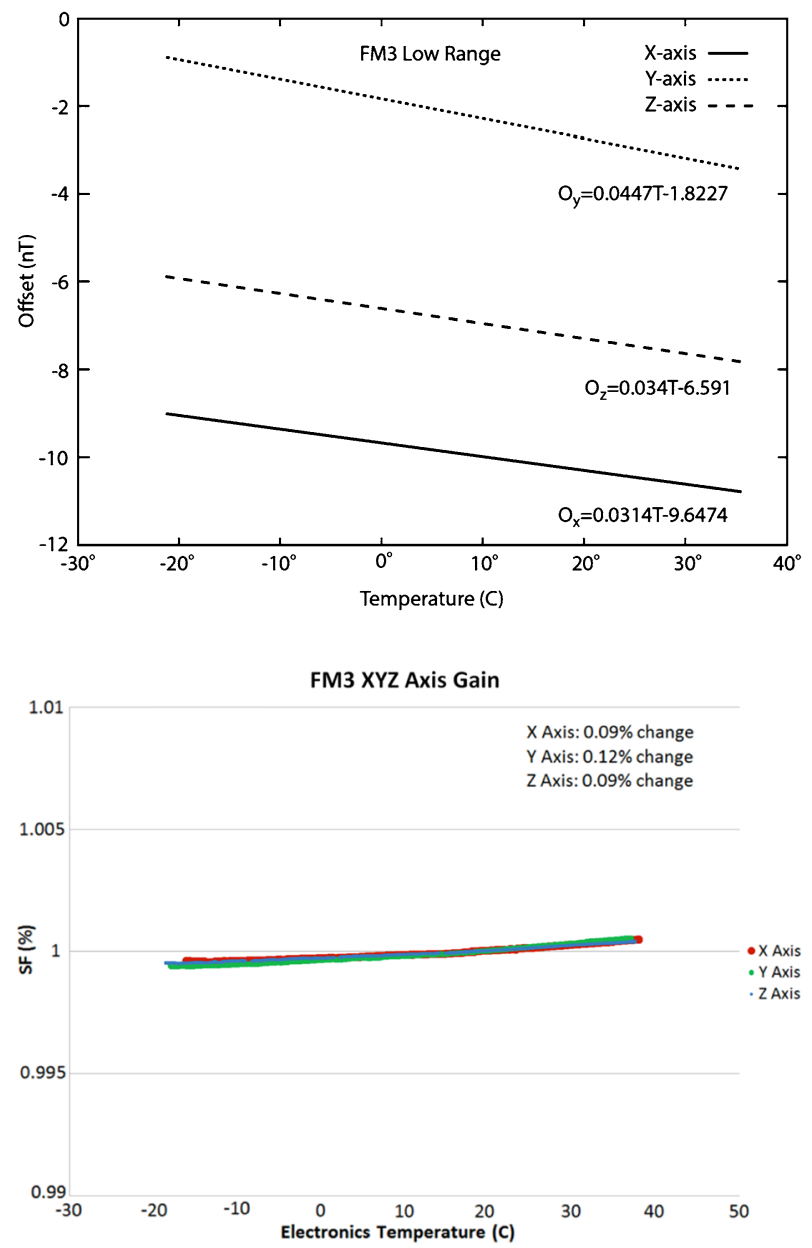

temperature range. The electronics unit temperature is monitored as part of the housekeeping data acquired during flight. Figure 24 shows the corresponding gain dependence, which is about $0.1 \%$ over the -20 to $+40{ }^{\circ} \mathrm{C}$ operating temperature range.

Figure 25 shows the gain variation as a function of temperature for the AFG FM3 sensor. This test used the shield-can test facilities at UCLA, which used the mu-metal shields to exclude the Earth's magnetic field, rather than a compensating coil system. The tests included a compensation factor to account for the change in the calibration coil characteristics as a function of temperature. The results for all the flight units were very similar and within specifications.

\subsubsection{Temperature Correction}

It is planned to use the measured temperature drifts of gains on ground to make corrections in-flight. Besides gain versus temperature, the ground calibration reports also contain orthogonality versus temperature as well as offset versus temperature. For spinning spacecraft, orthogonality can be well established in-flight also for varying temperatures (Leinweber 2012). It is expected that sensor offsets are mainly subject to random changes during the 


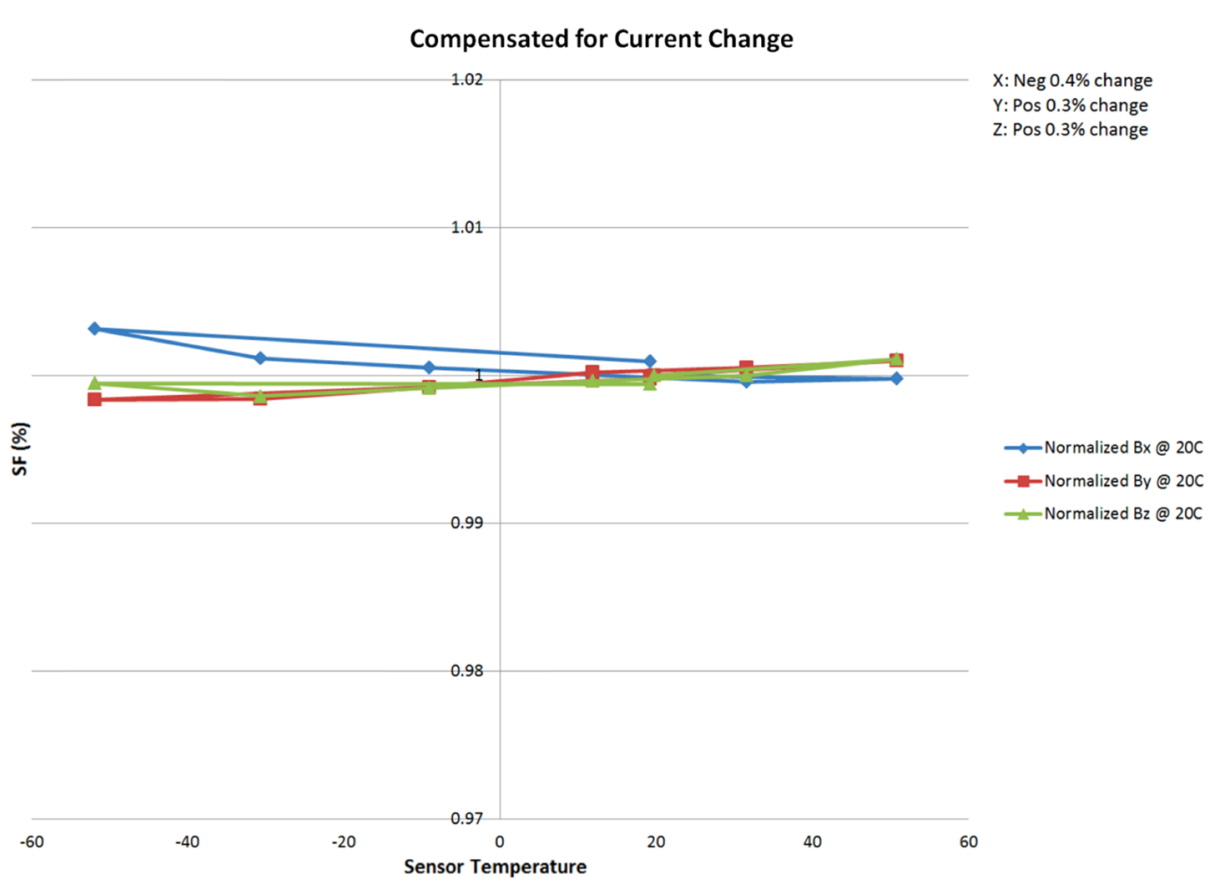

Fig. 25 AFG FM3 gain dependence on sensor temperature, relative to the gain at $20^{\circ} \mathrm{C}$

MMS mission. Gains will be corrected for temperature drifts post-launch. The corrections will be applied by using the temperature information from the housekeeping data streams and ground calibration results. Also, a temperature-dependent non-linearity correction will be applied to the DFG data.

\subsection{In-Flight Calibration}

The twelve calibration parameters of a linear magnetometer have been introduced at the beginning of this section. There are a number of different techniques that can be used to cross check or adjust magnetometer calibration parameters on orbit. The techniques that will be used for in-flight calibration of the MMS-magnetometers are described below. This section uses $(1,2,3)$ for sensor coordinates and $(x, y, z)$ for orthogonalized coordinates. The abbreviation " $S P$ " is used to address both spin plane components of an orthogonalized system.

Eight out of twelve calibration parameters can be found via removal of spin tone in a de-spun coordinate system. The remaining four calibration parameters are calculated using a set of techniques. The set consists of removal of jumps that occur during range changes, Earth field comparison, cross calibration with EDI, calibrations in the solar wind (MMS will rarely encounter the solar wind) and inter-spacecraft calibration. The overall calibration plan for producing the science-grade Level-2 data is outlined in Fig. 26.

\subsubsection{Spin Tone Removal (Orthogonalization)}

The orthogonalization procedure removes the spin tone that is left when magnetometer data are transformed from a spinning to a non-spinning coordinate system. This spin tone consists 
Fig. 26 Outline of the overall AFG/DFG calibration plan for science-grade Level-2 data

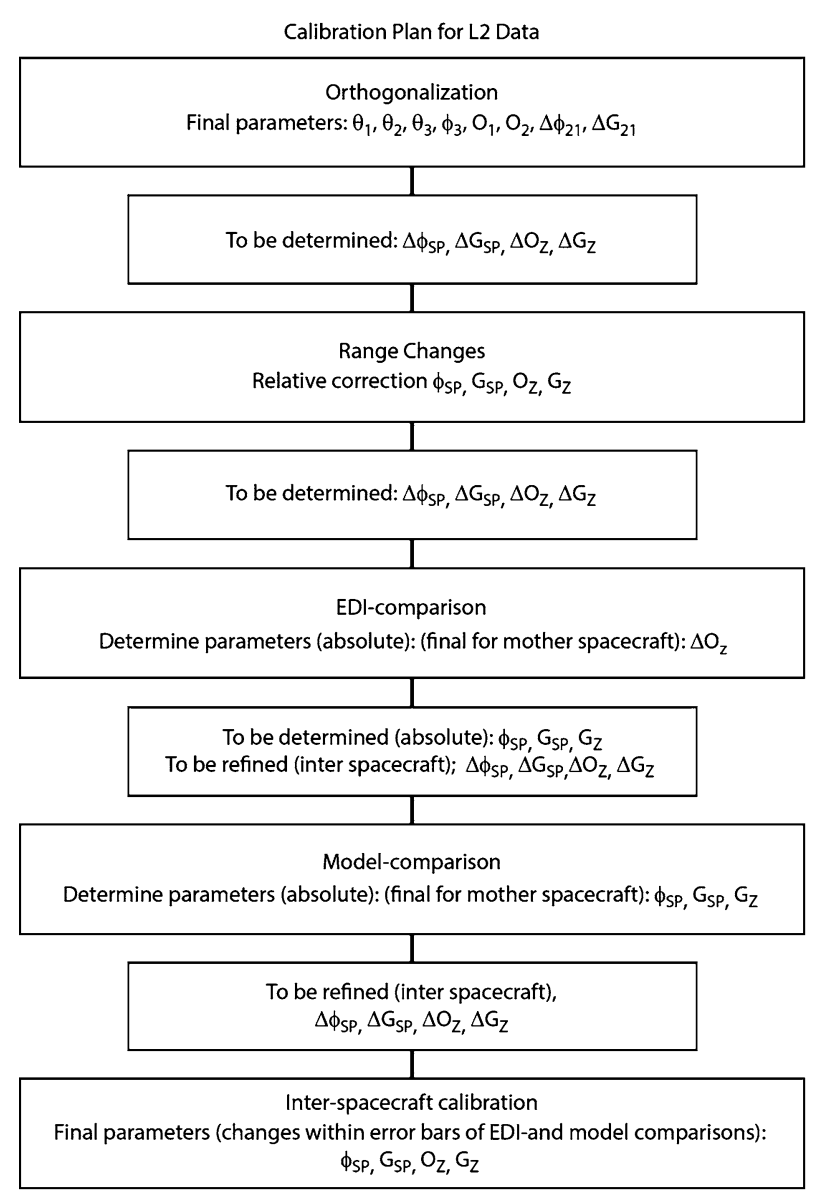

of first and second harmonics of the spacecraft spin frequency for the spin plane sensors and a first harmonic for the spin axis sensor. The harmonics can be linked to calibration parameters (Kepko et al. 1996).

1. First harmonics in the spin plane sensors are produced by miscalibrated $O_{1}, O_{2}, \theta_{1}$ and $\theta_{2}$.

2. Second harmonics in the spin plane sensors are produced by miscalibrated $\Delta G_{21}$ and $\Delta \varphi_{21}$, where $\Delta \varphi_{21}=\varphi_{2}-\varphi_{1}-90^{\circ}, \Delta G_{21}=G_{2}-G_{1}$ and $\Delta \varphi_{21}=\varphi_{2}-\varphi_{1}-90$, thus the second harmonics depend on the relative spin plane gains and the relative azimuthal angles of the spin plane sensors and are independent of their absolute values.

3. First harmonics in the spin axis sensor are produced by miscalibrated $\theta_{3}$ and $\varphi_{3}$.

The calibration parameters $O_{3}, G_{3}$, the absolute values $G_{1}, G_{2}$, the absolute values of $\varphi_{1}$ and $\varphi_{2}$ cannot be linked to spin harmonics. For the Cluster mission the amplitudes of the spin harmonics were estimated using Fourier Transforms (Kepko et al. 1996). This method has limitations when the amplitudes of the spin harmonics change within one window that is used to calculate Fourier coefficients. The Galileo spacecraft was spinning with a period of $\sim 20 \mathrm{~s}$ (similar to MMS) during its two Earth flybys. The spin harmonics changed too rapidly for the Fourier method. Another method for calculating spin harmonics for every 
Fig. 27 The first and second panels show magnetic field values that contain spin harmonics plus envelopes. The first panel shows the first harmonic of a despun component of the magnetic field after filtering. The second panel shows the second harmonic of the component after filtering. The bottom panel shows the amplitudes of the first and second spin harmonics as calculated from the envelopes

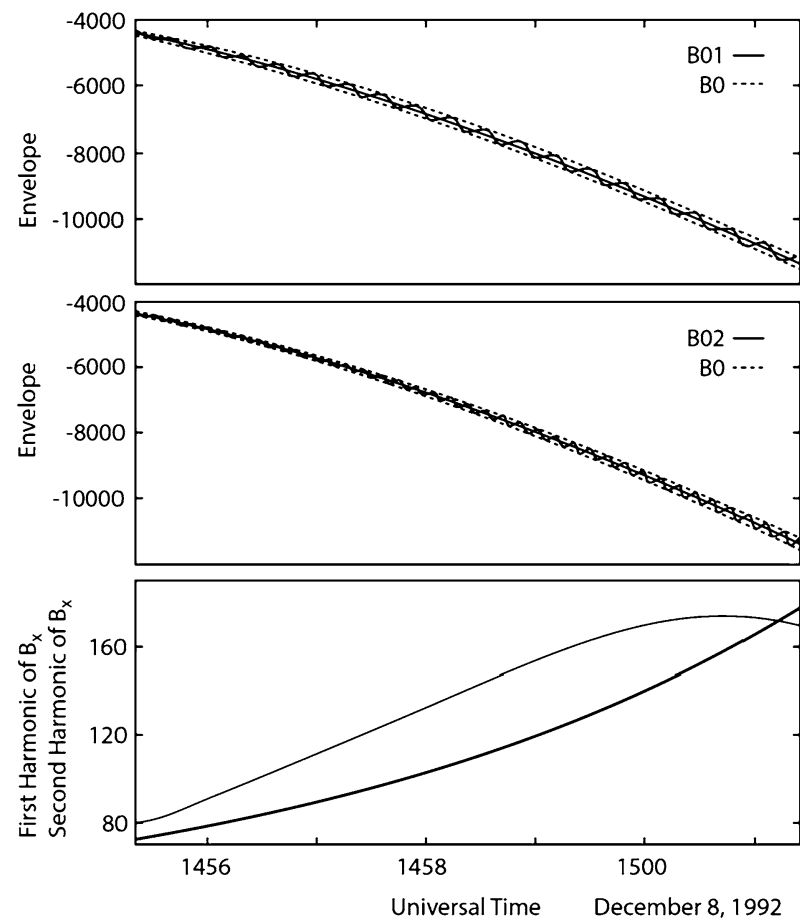

point in time was developed (Leinweber 2012). It calculates the spin harmonics for every point in time via envelopes (Envelope-Method). Figure 27 shows an example of spin harmonics derived from envelopes. The first harmonics are calculated using a filter that removes the second harmonics. The second harmonics are calculated using a filter that removes the first harmonics. Zero-phase filters must be used.

The Envelope method and a modified FFT method that uses short-time Fourier transforms are available for MMS. The Fourier based method can be used at times when it is difficult to calculate envelopes. The Envelope-Method can be used for fast changing spin harmonics. Fast changing spin harmonics are expected to be observed close to perigee where it is easy to calculate envelopes due to low geophysical noise compared to the magnetic field strength. The stability and error estimates of the calculated calibration parameters will be determined using a Blocked Bootstrap Monte Carlo Simulation.

At times when the spacecraft emerges from an eclipse, large changes of temperature arise, thus calibration parameters must be adjusted with higher time resolution. For the Cluster mission, we developed an orthogonalization method that uses sophisticated running averaging procedures to find the calibration parameters with higher time resolution. The basic principle of the method is that the contributions of the first harmonics become constants in a frame that spins with the spacecraft's spin frequency. Similarly, the second harmonics become constants in a frame that spins with twice the spin frequency. Running averages in appropriate coordinate systems make it possible to adjust calibration parameters for orthogonalization with high enough time resolution, so that no spin tone is left in the data that are measured shortly after eclipses. More details can be found in Leinweber (2012). 


\subsubsection{Removal of Jumps During Range Changes}

After orthogonalization calibration parameters that were impossible to determine via spin tone removal can be used, to remove jumps between range changes (Leinweber 2012). The jumps can be removed for the spin plane sensors by changing their gain and their azimuthal angle. The same gain and angle is applied to both spin plane sensors. The gain correction is applied to the lower range and the angle correction is applied to the higher range. For the spin axis a change of the zero level and a gain change can be used to remove jumps. The gain correction is applied to the lower range and the zero level correction is applied to the higher range. Information from several jumps is combined and a least squares solution, to derive the calibration parameters is performed. Combination of several jumps yields a range of input data which is required to derive stable solutions. Especially combining range changes at increasing fields with range changes at decreasing fields is important, since those range changes occur at different levels of the field.

Spin Plane The spin plane magnetic fields of the lower range are denoted as $B_{P L x}$ and $B_{P L y}$. Similarly for the upper range: $B_{P H x}$ and $B_{P H y}$. The subscript $P$ means "prime" and is used to denote uncorrected values. The subscript " $S P$ " denotes the spin plane sensors.

First calculating the gain change for both spin plane sensors

$$
\Delta G_{S P}=\left(B_{P H x}^{2}+B_{P H y}^{2}\right)^{1 / 2} /\left(B_{P L x}^{2}+B_{P L y}^{2}\right)^{1 / 2}
$$

Second calculating the change of azimuthal angle for both spin plane sensors

$$
\Delta \varphi_{S P}=\tan ^{-1}\left[\frac{\Delta G_{S P} B_{P H x} B_{P L y}-\Delta G_{S P} B_{P H x} B_{P L y}}{\Delta G_{S P}^{2} B_{P L x} B_{P L y}+B_{P H x} B_{P H y}}\right]
$$

The changes are applied as follows:

- Upper range:

$$
\left(\begin{array}{c}
B_{H x} \\
B_{H y}
\end{array}\right)=\left(\begin{array}{cc}
\cos \Delta \varphi_{S P} & -\sin \Delta \varphi_{S P} \\
\sin \Delta \varphi_{S P} & \cos \Delta \varphi_{S P}
\end{array}\right)\left(\begin{array}{c}
B_{P H x} \\
B_{P H y}
\end{array}\right)
$$

- Lower range:

$$
\left(\begin{array}{l}
B_{L x} \\
B_{L y}
\end{array}\right)=\left(\begin{array}{cc}
\Delta G_{S P} & 0 \\
0 & \Delta G_{S P}
\end{array}\right)\left(\begin{array}{l}
B_{P L x} \\
B_{P L y}
\end{array}\right)
$$

Spin Axis The magnetic field of the uncorrected lower range spin axis sensor is denoted as $B_{P L z}$. Similarly for the upper range: $B_{P H z}$. The subscript $z$ denotes the spin axis sensor.

The following equation can be solved in the least squares sense:

$$
B_{P L z} \Delta G_{z}=B_{P H z}-\Delta O_{z}
$$

The changes are applied as follows:

- Upper range:

$$
B_{H z}=B_{P H z}+\Delta O_{z}
$$

- Lower range:

$$
B_{L z}=\Delta G_{z} B_{P L z}
$$


For MMS the corresponding second magnetometer that is on the same spacecraft can be used to get better estimates of magnetic field values right before and after the range changes. The magnetometers AFG and DFG switch ranges at different magnetic field values. The stability and error estimates of the calculated calibration parameters will be determined using a Bootstrap Monte Carlo Simulation. Note that the delta gains are close to one, whereas the delta angle and delta offsets are close to zero.

\subsubsection{Determination of Spin Axis Offsets with EDI}

Each MMS spacecraft features two oppositely positioned gun-detector-units, belonging to the electron drift instrument (EDI), that emit and detect returning electron beams. Both, electron time-of-flight (TOF) and beam firing direction (BD) data can be used to determine AFG and DFG spin axis offsets $\left(O_{z}\right)$.

The TOF of the electron beams is inversely proportional to the ambient magnetic field strength. However, as discussed in Nakamura et al. (2014), TOF measurements by EDI are themselves subject to offsets. These offsets can be determined by comparison to spin plane magnetic field measurement by AFG or DFG, whose modulus is unaffected by spin axis offsets. Once EDI TOF offsets are corrected, differences in strength of spin axis directed magnetic fields, as measured by AFG/DFG and given by EDI TOFs, then yield AFG and DFG spin axis offsets (Georgescu et al. 2006; Leinweber et al. 2013; Nakamura et al. 2014).

Furthermore, gun-detector-units have to emit electron beams in directions that are perpendicular to the magnetic field for successful return. Inaccuracies in spin axis offsets lead to deviations from $90^{\circ}$ in the angles $\alpha$ between BDs and AFG/DFG determined magnetic field vectors, in particular if these vectors have a major spin plane component. Hence, AFG and DFG spin axis offsets can be determined by minimization of these deviations. Any comparison of BDs and magnetic field vectors needs to be performed in a common system of reference. Transformations into such a system can be decisively improved in accuracy by using EDI and magnetometer measurements for which $\alpha$ is unaffected by the spin axis offset (BD in the spin plane).

The offset estimates from both TOF and BD methods can be combined to yield a single, average offset for a specific time interval, although the methods are complementary: The TOF method is sensitive to changes in the measured magnetic field strength, which in turn is most affected by the spin axis offset if the field is directed toward the spin axis. The BD method, instead, is sensitive to changes in measured magnetic field direction, which is most dependent on spin axis offset if the magnetic field is lying in the spin plane. More detailed descriptions of both TOF and BD methods can be found in Plaschke et al. (2014).

\subsubsection{Calibration in the Solar Wind}

The four MMS spacecraft will encounter the interplanetary magnetic field only during unusual solar wind conditions. Thus, EDI is expected to be the main source for calculating spin axis offsets $\left(O_{z}\right)$. However, when unusual conditions bring MMS into the interplanetary magnetic field, zero levels can be calculated via the property that fluctuations of the interplanetary field are primarily changes in the direction rather than in the magnitude so that the field magnitude is more constant than any of its three component axes (Ness et al. 1964). Incorrect zero levels (offsets) lead to an increase of the fluctuations of the field magnitude. However, compressional fluctuations can appear naturally in the solar wind, too. MMS will observe the interplanetary magnetic field close to the bow shock during these unusual solar wind conditions with higher than average dynamic pressure values where there 
are non-Alfvénic upstream waves. Thus, use of the Alfvenicity of the interplanetary magnetic field needs to be accompanied by checks of the stability of the calculated offsets. The method used employs error estimates using a Blocked Bootstrap Monte Carlo Simulation. The method is described in Leinweber et al. (2008). It is a modified Davis-Smith method that determines magnetometer zero levels on short time scales and it has a sophisticated algorithm to distinguish Alfvénic intervals from non-Alfvénic intervals of input data.

\subsubsection{Earth Field Comparison}

Removal of spin harmonics cannot resolve four of the calibration parameters. These parameters are the absolute value of the azimuthal angles $\varphi_{S P}$, the absolute value of the spin plane gains $G_{S P}$, the absolute value of the spin axis gain $G_{z}$ and the spin axis offset $O_{z}$. We note that Earth field models are usually not accurate enough to solve for magnetometer zero levels. The removal of the jumps at the range changes matches the upper and the lower ranges. Thus the calibration parameters that are obtained at perigee in high range can be applied to both ranges, the high and the low.

For resolving calibration parameters, the model, as well as the data, need to be transformed into orthogonal despun spacecraft coordinates (Leinweber 2012).

$$
\left(\begin{array}{l}
B_{m x} \\
B_{m y} \\
B_{m z}
\end{array}\right)=\left(\begin{array}{ccc}
\cos \varphi_{S P} & \sin \varphi_{S P} & 0 \\
-\sin \varphi_{S P} & \cos \varphi_{S P} & 0 \\
0 & 0 & 1
\end{array}\right)\left(\begin{array}{ccc}
G_{S P} & 0 & 0 \\
0 & G_{S P} & 0 \\
0 & 0 & G_{z}
\end{array}\right)\left(\begin{array}{l}
B_{s c x} \\
B_{s c y} \\
B_{s c z}
\end{array}\right)
$$

where $B_{m x}, B_{m y}, B_{m z}$ is the model field in orthogonal despun spacecraft coordinates and $B_{s c x}, B_{s c y}, B_{s c z}$ measured field in pseudo orthogonal despun spacecraft coordinates

The above equation can be split into two parts.

$$
\left(\begin{array}{cc}
B_{s c x} & B_{s c y} \\
B_{s c y} & -B_{s c x}
\end{array}\right)\left(\begin{array}{c}
G_{S P} \cos \varphi_{S P} \\
G_{S P} \sin \varphi_{S P}
\end{array}\right)=\left(\begin{array}{c}
B_{m x} \\
B_{m y}
\end{array}\right)
$$

and

$$
B_{s c z} G_{z}=B_{m z}
$$

The calibration parameters are calculated as follows:

$$
\begin{aligned}
\varphi_{S P} & =\tan ^{-1}\left(\frac{G_{S P} \sin \varphi_{S P}}{G_{S P} \cos \varphi_{S P}}\right) \\
G_{S P} & =\sqrt{\left(G_{S P} \cos \varphi_{S P}\right)^{2}+\left(G_{S P} \sin \varphi_{S P}\right)^{2}} \\
G_{z} & =\frac{B_{m z}}{B_{s c z}}
\end{aligned}
$$

For MMS the expected maximum field magnitude at perigee is $\sim 8000 \mathrm{nT}$. Model parameters such as the ring current index Dst can make a significant change to the field strength at MMS perigee. Many perigee passes will be combined to provide more accurate estimates of calibration parameters. Additionally perigee passes that occur during extreme solar wind conditions will be dismissed from calculations. The stability and error estimates of the calculated calibration parameters will be determined using a Blocked Bootstrap Monte Carlo Simulation. 
Fig. 28 Blue: best effort calibration; Red: best effort calibration plus inter-spacecraft calibration. The top panel shows a comparison of current density before and after inter-spacecraft calibration. The second panel shows a comparison of divergence before and after inter-spacecraft calibration. The third panel shows $B_{x}$ (the solar oriented component) and the bottom panel shows the inter-spacecraft distances. The three traces are largest, intermediate and smallest spacecraft distance. Here the intercalibration step makes very little difference
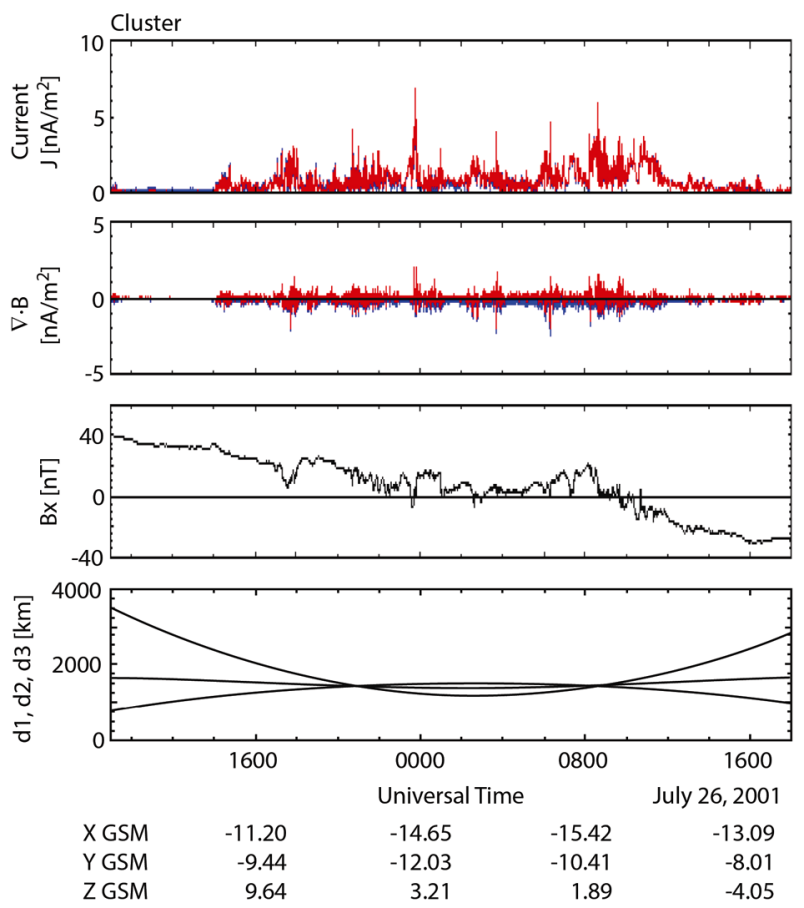

\subsubsection{Inter-spacecraft Calibration}

Inter-spacecraft calibration for MMS will mainly focus on adjusting four calibration parameters that cannot be determined from orthogonalization $\left(\Delta \varphi_{S P}, \Delta G_{S P}, \Delta G_{z}\right.$ and $\left.\Delta O_{z}\right)$. For inter-calibration we chose a "mother" spacecraft for which we do not further change the calibration parameters. The four parameters for each of the other three spacecraft will be changed relative to the mother spacecraft, within the error estimates of the EDI calibration, solar wind calibration (if applicable) and Earth field calibration. For the Cluster mission curl and divergence were minimized inside quiet regions of the tail lobes, where these quantities are expected to be vanishingly small (Khurana et al. 1996; Leinweber 2012; Leinweber et al. 2013). For accurate calculation of spatial gradients, the importance of interspacecraft calibration increases with decreasing spacecraft separations (Leinweber et al. 2013).

Figure 28 depicts data from a Cluster plasma sheet crossing with $\sim 1000 \mathrm{~km}$ interspacecraft spacing. The first and second panels show the curl and the divergence calculated from first order Taylor series expansion (Khurana et al. 1996). The blue traces are calculated with all other calibrations except inter-spacecraft calibration. The red traces include interspacecraft calibration. The third panel shows the $x$-component of the magnetic field of a typical plasma sheet crossing as observed by Cluster. The fourth panel shows the maximum, intermediate and minimum inter-spacecraft distances. Figure 29 depicts data from a Cluster plasma sheet crossing with $\sim 100 \mathrm{~km}$ inter-spacecraft spacing. It is obvious that the latter figure shows much greater differences between intercalibrated and non-intercalibrated data. For MMS we expect to decrease the inter-spacecraft distances down to $\sim 10 \mathrm{~km}$. This can be expected to lead to dramatic differences between intercalibrated and non-intercalibrated spatial gradients. 
Fig. 29 Blue: best effort calibration; Red: best effort calibration plus inter-spacecraft calibration. The top panel shows a comparison of current density before and after inter-spacecraft calibration. The second panel shows a comparison of divergence before and after inter-spacecraft calibration. The third panel shows $B_{x}$, the solar pointing component, and the bottom panel shows the inter-spacecraft distances. The three traces are largest, intermediate and smallest spacecraft distance. Here, in contrast to Fig. 28, the intercalibration has made a large difference because the real currents occur in thin layers and the uncalibrated differences between spacecraft are 'divided by' a smaller distance

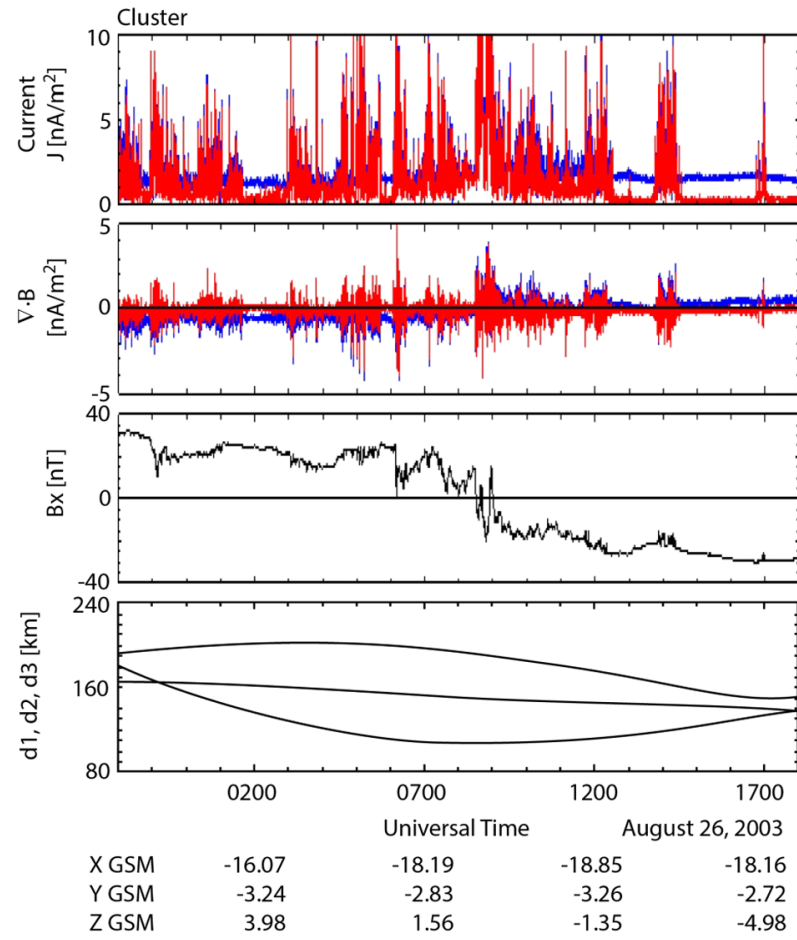

MMS will not travel through the tail lobes for large portions of its mission where curl and divergence of the magnetic field are expected to become small. Thus, MMS inter-spacecraft calibration will primarily focus on optimizing the divergence of the magnetic field using a subset of the equations published in Leinweber (2012), Leinweber et al. (2012). When possible, divergence as well as curl of the magnetic field will be minimized (inside of the tail lobes). An additional constraint that must be taken into consideration is the closeness of the tetrahedron to being a regular tetrahedron. Inter-spacecraft calibration as described herein requires the tetrahedron to be close to being regular and cannot be performed at regions where it is not. The stability and error estimates of the calculated calibration parameters will be obtained by a Blocked Bootstrap Monte Carlo Simulation. Expected errors of the interspacecraft distances can be neglected compared to the expected errors of the magnetic field gradients (Leinweber et al. 2013).

\subsubsection{Combination of Calibration Parameters}

The overall in-flight calibration process is a shared effort between different individuals working at different institutions. The calibration process is outlined in a way that it comprises a step by step process without circularity. After establishing orthogonality, changing the four remaining parameters has no effect on spin harmonics in the despun coordinate system. Let $\mathbf{C}^{\text {orth }}$ and $\mathbf{O}^{\text {orth }}$ be the calibration matrix and offset vector after orthogonalization. Equations (15a), (15b) gives an example on how changes of $\varphi_{S P}, G_{S P}, G_{z}$ and $O_{z}$ can be applied to an existing calibration matrix and offset vector with $\mathbf{C}^{\text {adj }}$ being the matrix that must be applied to the existing calibration. A small compromise is being made in order to avoid circularity of the calibration process by applying the changes in the orthogonalized 
system:

$$
\begin{aligned}
\mathbf{C}^{a d j} & =\left(\begin{array}{ccc}
\cos \Delta \varphi_{s p} & \sin \Delta \varphi_{s p} & 0 \\
-\sin \Delta \varphi_{s p} & \cos \Delta \varphi_{s p} & 0 \\
0 & 0 & 1
\end{array}\right)\left(\begin{array}{ccc}
G_{s p} & 0 & 0 \\
0 & G_{s p} & 0 \\
0 & 0 & G_{z}
\end{array}\right) \\
\mathbf{C}^{\text {new }}+\mathbf{O}^{\text {new }} & =\mathbf{C}^{\text {adj }} \mathbf{C}^{\text {orth }}+\mathbf{C}^{\text {adj }} \mathbf{O}^{\text {orth }}+\left(\begin{array}{c}
0 \\
0 \\
O_{z}
\end{array}\right)
\end{aligned}
$$

\subsubsection{Archiving of Calibration Parameters}

If no archiving of calibration results is done, all that is left from the calibration work is a set of changing calibration parameters. If at a later time, questions about the accuracy of the calibration parameters for a certain time interval arise, no answers can be provided and the time interval in question must be reevaluated or recalibrated in order to address the questions. At the time of writing, an archiving system for MMS-AFG and -DFG calibration parameters is under discussion. Ideally, such an archiving system provides tools that are easy to use for obtaining the following information:

- Calibration parameters and status of parameters

- Valid time intervals of calibration parameters

- Input time intervals and data that were used to calculate the calibration parameters

- Kind of calibration (algorithm, version of algorithm)

- Error estimates

- What data were produced with the calibration parameters

- Special relevant occurrences (eclipses, space weather events)

- Documentation

It is also important to note that the data files should contain a link to the applied calibration parameters. This has not always been done on previous projects, preventing a determination of why different authors or the same author at different times have obtained different results. As Figs. 28 and 29 show, the results of our differencing operators depend sensitively on the accurate intercalibration of the data.

\section{Magnetic Cleanliness Program}

The first half of this paper has described how to obtain accurate measurements of the local magnetic field. However, the objective of the mission is to obtain accurate measurements of the ambient magnetic field in the plasma which could be masked in part by spacecraft fields. Moreover, the spacecraft fields could affect more measurements than just those of the magnetotometer. To achieve the mission objectives, the magnetic fields generated by the spacecraft must corrupt neither the measurement of the natural magnetic fields nor the trajectories of electrons whose directions of arrival are critical to two other instruments. Because of the high precision requirements for inter-comparison of the magnetic field between MMS spacecraft, the contamination magnetic fields were required to be very stable. Moreover, assuring that electrons are not deflected by spacecraft magnetic fields, imposes stringent limits on the allowed maximum contamination field near the spacecraft body. Each of the four MMS spacecraft hosts a large number of avionics sub-systems, instruments, and 
deployables provided by more than twenty institutions and vendors. In addition, the dynamics of the power system current with spacecraft spin imposed tight constraints on power system magnetics. These factors required a comprehensive but efficient program of magnetics design assessment and guidance, verification, and testing, that could be conducted remotely or with transportable test equipment in facilities not designed to be magnetically clean. Moreover, the MMS observatories were too large to be accommodated in the Acuña magnetics test facility at GSFC, so tests for variable and static spacecraft magnetic fields had to be devised that were suitable for conventional integration facilities.

\subsection{Magnetics Cleanliness Requirements}

The MMS science objectives and corresponding measurement requirements impose upper limits on magnetic fields generated by the MMS spacecraft as summarized in Table 3 . The measurement requirements of both the fluxgate (FGM) and search coil (SCM) magnetometers dictate the maximum allowed spacecraft magnetic signals at the locations of the search coil sensor, 4 meters outboard on one boom, and the two fluxgate sensors, 5 meters outboard on each of two opposing booms. The MMS mission objectives require precision intercomparison accurate to $0.1 \mathrm{nT}$ between magnetic fields at the four observatories, implying the fixed field (DC) stability requirement at the locations of the fluxgate sensors. This stability requirement applies throughout the period of time, estimated to be 4000 seconds in duration each orbit, that the four spacecraft transit the target regions where magnetic reconnection occurs. To ensure that the magnetometers accurately specify the background field, the total spacecraft background field at the sensors must not exceed $10 \mathrm{nT}$ and this is adopted as the total fixed field (DC) upper limit. These requirements were imposed for the MMS observatories in science observation mode, and fast survey mode in particular. There was no requirement to comply with the magnetic emission limits during boom deployments, thrusting, through Earth eclipses, or other operations that do not occur during science observations. The transponder transmitter will be powered on and off during science observations so the magnetic field associated with transmission on/off was applied against the field DC stability requirement for the transponder.

Magnetic signatures of low frequency waves and turbulence are another key observation objective and the fluxgate instruments are designed to provide continuous sampling from DC to $64 \mathrm{~Hz}$ Nyquist. The search coil instrument provides sampling from 1 to $6 \mathrm{kHz}$. The frequency response functions of the fluxgate a search coil instruments were designed to optimize sensitivity over these frequency ranges. Their sensitivities are shown in Fig. 30. The lower envelope of the instrument response functions defined the limit for spacecraft magnetic field emissions. Conventional electromagnetic interference (EMI) testing applies above $10 \mathrm{~Hz}$ and these requirements were conveyed to the engineering teams. The requirements below $10 \mathrm{~Hz}$ were assigned to the magnetics cleanliness effort. To ensure overlap with established EMI testing, the customized magnetics testing program adopted the frequency response from 0.02 to $30 \mathrm{~Hz}$ as the low frequency magnetic field requirement for the observatory.

In addition to the direct sensitivity of the magnetic field measurements, the Electron Drift Instrument (EDI) and the Dual Electron Spectrometer (DES) measurements can also be adversely affected by contamination magnetic fields. The two heads of EDI, the Gun Detector Units (GDU), located on diametrically opposite faces of the MMS observatory, fire $\sim 1 \mathrm{keV}$ electron beams in directions which are steered to return to the opposing EDI-GDU head. Determination of the electric field and magnetic field magnitude from the observed electron drift depends on the assumption that the spacecraft magnetic field does not appreciably 
Table 3 Maximum allowed magnetic fields generated by the MMS spacecraft

\begin{tabular}{llccc}
\hline Sensor & Requirement & $\begin{array}{l}\text { Magnetic field } \\
\text { limit (nT) }\end{array}$ & $\begin{array}{l}\text { Nominal } \\
\text { distance (m) }\end{array}$ & $\begin{array}{l}\text { Nominal } \\
\left.\text { moment (A-m }{ }^{2}\right)\end{array}$ \\
\hline $\begin{array}{l}\text { FGM: Fluxgate } \\
\text { magnetometers }\end{array}$ & Total field & 10.0 & 5 & 6.25 \\
FGM & $\begin{array}{l}\text { Magnetic field } \\
\text { stability }\end{array}$ & 0.1 & 5 & 0.06 \\
$\begin{array}{l}\text { EDI: Electron drift } \\
\text { instrument }\end{array}$ & $\begin{array}{l}\text { Radial total magnetic } \\
\text { field } \\
\text { EDI }\end{array}$ & 24 & 1.5 & 0.41 \\
$\begin{array}{l}\text { Tangential total } \\
\text { magnetic field } \\
\text { TES: Dual electron } \\
\text { sensor }\end{array}$ & 42 & 1.5 & 0.71 \\
\hline
\end{tabular}

${ }^{\text {a }}$ Requirements on the total field and on the stability of the spacecraft-generated field derive from different science measurement objectives. Different sensors or instruments imposed limits at the locations of each on either the total field at the distances from the nearest portion of the observatory as noted. The moment limit corresponds to the moment that would generate the upper limit field at the nominal distance listed. In the case of EDI the upper limit deflection of $0.25^{\circ}$ for a $1 \mathrm{keV}$ electron was used as a refinement on these nominal upper limits to facilitate magnetic moment allocations

deflect the electron beam. Hence, the EDI instrument imposes constraints on the magnetic field near the body of the spacecraft in the vicinity of the EDI-GDU units as listed in Table 3 . Additional analyses of $1 \mathrm{keV}$ electron beam deflection were conducted to establish the upper limits for magnetic moments as functions of position on the spacecraft relative to the EDI location that would ensure deflection by less than $0.25^{\circ}$. Similarly, the DES angle of arrival measurement will be corrupted if the incoming electrons are deflected by spacecraft magnetic fields and the upper limit magnetic field near the DES sensor is also listed in Table 3.

\subsection{Allocation Approach}

The MMS observatory diameter is 3.4 meters from corner to corner, so the moment limits given in Table 3 are not useful for understanding the allowed moments of units on the spacecraft. More detailed analyses were done to derive magnetic moment limits appropriate for the actual locations of each unit to guide design and testing during development. Moreover, the limit for the observatory contamination magnetic field was divided across all of the units in the observatory to arrive at allocations for each unit so that the integrated observatory would comply with the contamination magnetic field limits. We first determined the maximum allowed moment of each magnetic field source allowed within the most restrictive magnetic field requirement for the source location and then assigned a fraction of this moment as the source allocation. The approach we adopted provided avoided excessive conservatism while specifying criteria to identify sub-systems requiring mitigation.

\subsubsection{Source-Victim Analysis and Root-Sum-Square Allocation}

Considering each sub-system element as a magnetic field source and denoting the two fluxgate sensors, search coil sensor, four DES detectors, and two EDI units as the set of magnetics victims, we first specified the locations of all sources and victims in spacecraft coordinates. The maximum possible magnetic field at the $j$ th victim due to the $i$ th source with a 


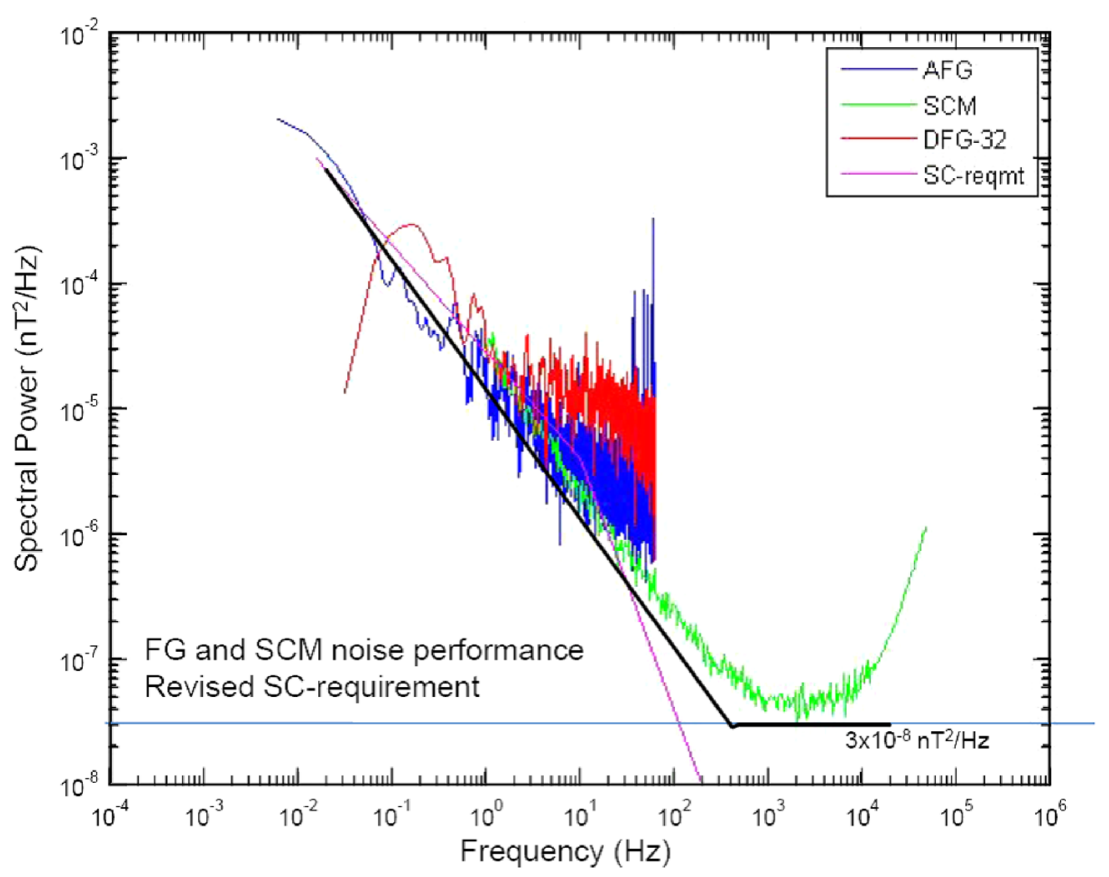

Fig. 30 Sensitivities of the FGM and SCM units expressed in power spectral density. The black solid line shows the observatory emission requirement at the locations of the FGM and SCM sensors adopted for MMS

magnetic moment magnitude $\mu_{i}$ separated by distance $D_{i, j}$ is

$$
B_{i, j}=\frac{\mu_{0}}{2 \pi} \frac{\mu_{i}}{D_{i, j}^{3}}
$$

where it has been assumed that the moment is directed along the line between the source and victim, that is, the worst-case moment orientation. The relationship between the magnetic field requirement for the $j$ th victim, $B_{\mathrm{Req}, j}$, or magnetic stability, $\Delta B_{\mathrm{Req}, \mathrm{Stab}}$, and the corresponding upper limit magnetic moment of the $i$ th unit, $\mu_{\text {Limit }, i}$, is

$$
\begin{aligned}
\mu_{\text {Limit }, \mathrm{DC}, i} & =\min \left\{\frac{B_{\mathrm{Req}, j}}{200} D_{j, i}^{3}\right\} \\
\mu_{\mathrm{Limit}, \mathrm{Stab}, i} & =\frac{\Delta B_{\mathrm{Req}, \mathrm{Stab}}}{200} D_{i}^{3}
\end{aligned}
$$

where (17a) and (17b) are for the total field and the field stability, respectively, $B$ and $\Delta B$ are in $\mathrm{nT}, D$ is in meters, and $\mu_{\text {Limit }}$ is in A-m ${ }^{2}$. In (17a) the minimum is taken over all of the victims. Note that $\mu_{\text {Limit }}$ corresponds to the entire spacecraft magnetic contamination allowance.

The next step in the analysis is to calculate the appropriate fraction of $\mu_{\text {Limit }, i}$ yielding the moment allocation to the $i$ th unit, $\mu_{\mathrm{Alloc}, i}$, such that the observatory limit for $\Delta B_{\mathrm{Req}, j}$ would not be exceeded even if all units were to generate their allocated moment. If one knew the vector moment of each source one could calculate its contribution to the magnetic field at the $j$ th victim, $\mathbf{B}_{i, j}$ and the total field would be the magnitude of the summed vector fields 
Fig. 31 Allocation root-sum squares confidence test. Black trace (left axis) shows the occurrence distribution of the vector field magnitude divided by the root-sum-square of 50 component, equal magnitude vectors. Red trace (right axis) is the cummulative probability showing that $65 \%$ of cases have $B_{\text {Total }} / B_{\text {Total }}^{*}<1$ and $92 \%$ have $B_{\text {Total }} / B_{\text {Total }}^{*}<1.5$

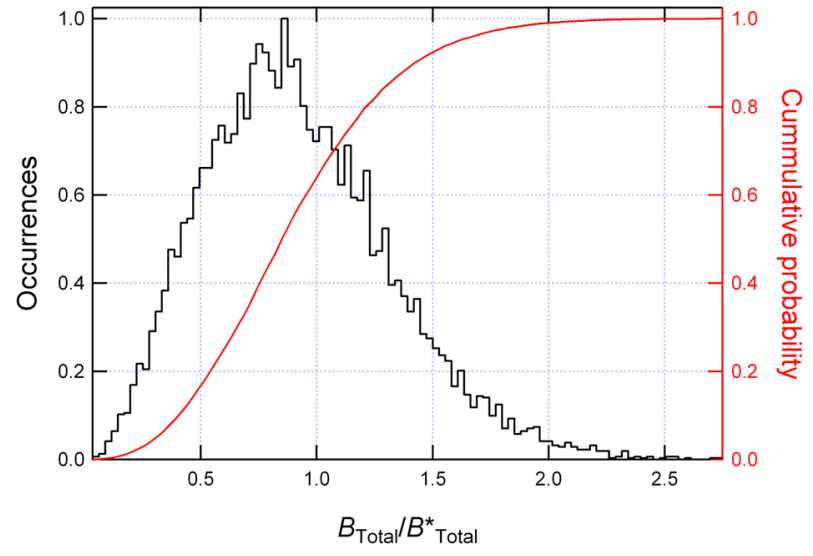

at the $j$ th victim:

$$
B_{\text {Total }, j}=\sqrt{\left(\sum B_{x, i, j}\right)^{2}+\left(\sum B_{y, i, j}\right)^{2}+\left(\sum B_{z, i, j}\right)^{2}}
$$

Of course, until the spacecraft and all subsystem units are actually designed and tested one cannot estimate $B_{\text {Total }, j}$, because one does not know the actual magnetic moments of each source. To provide useful guidance to the design engineers, one must therefore work backwards from $B_{\text {Total, } j}$ to calculate allocations to sub-systems. We approximated $B_{\text {Total }, i}$ as the root-sum-square (RSS) of the magnitude of the field contribution allocated to each source, $B_{\text {Alloc }, i, j}$. That is,

$$
B_{\mathrm{Total}, j}^{*}=\sqrt{\sum B_{\mathrm{Alloc}, i, j}^{2}}
$$

To estimate the degree of confidence that a design to $B_{\text {Total, } j}^{*}$ will result in a net field, $B_{\text {Total }, j}$, within the requirements we performed the following simple calculation. Taking 50 equal magnitude vectors we computed $B_{\text {Total }}$ and $B_{\text {Total }}^{*}$ for 10,000 different random relative orientations of the vectors and computed $B_{\text {Total }} / B_{\text {Total }}^{*}$ for each orientation set. Figure 31 shows the occurrence distribution of $B_{\text {Total }} / B_{\text {Total }}^{*}$ together with the accumulated probability distribution. In $64 \%$ of the runs, $B_{\text {Total }} / B_{\text {Total }}^{*}<1$, and in $92 \%$ of cases, $B_{\text {Total }} / B_{\text {Total }}^{*}<1.5$. The distribution is independent of the number of vectors since it is nothing more than the distribution of a three-dimensional random walk. Given the conservatism already implicit in selecting the tightest moment constraint for each unit (Fig. 31), the judgment was made to require $B_{\text {Total }} / B_{\text {Total }}^{*}<1$. In practice, the moments for most sources were smaller than their allocations by a factor of over 5 so that the risk of exceeding $B_{\text {Req,j }}$ using the root sum square (RSS) approximation turned out to be low.

The unit allocations were then computed by taking the moment limit from each source in (17a), (17b) and dividing this by the square root of the number of units, $N_{\text {Units, }}$, times a magin factor, $f_{\text {Margin }}$, so that the allocation for the $i$ th unit was given by

$$
\mu_{\mathrm{Alloc}, i}=\mu_{\mathrm{Limit}, i} / \sqrt{f_{\text {Margin }} N_{\mathrm{Units}}}
$$

To provide $\sim 20 \%$ margin, $f_{\text {Margin }}$ was set to 1.2 . Note that for 50 units the RSS approach provides a factor of seven less conservatism than an alternative worst case scalar sum of the 
total field magnitudes, but provides high confidence in meeting the design requirement. For the MMS spacecraft, there were 59 units with steady moments and 49 units with variable moments. These include: all solar panels, all electronics units, all propulsion thrusters and valves, the battery, and deployables. The spacecraft structural materials and fasteners were specified to be non-magnetic. In some instances, most notably the battery cell casings, nonpermeable alternatives were not available and these were screened and degaussed to achieve negligible permanent magnetic moments (see also Sect. 5.3.1 below).

\subsubsection{Unit-Level Allocation Division}

Three contributions to magnetic emissions of each unit were considered. They were: current loops, permanent magnets, and magnetized permeable materials. Since these moments are uncorrelated in direction, the unit moment allocation was divided using an RSS approach between the relevant contributors for each unit. Most units contained no permanent magnets and for these units, the allocation to current loops and materials were each assigned as $\mu_{\text {Alloc }, i} / \sqrt{2}$. For units such as thrusters, valves, or deployment mechanisms which carry no electric current during science operations, the allocation was assigned entirely to materials and magnets. For units that had all three types of sources the allocation assigned to each was $\mu_{\mathrm{Alloc}, i} / \sqrt{3}$. In some cases, one source turned out to exceed its portion of the allocation whereas another did not. In such cases, the allocation was reassigned in an RSS sense, for example for a unit with two types of sources, one was allowed a fraction, $f$, and the other $\sqrt{\left(1-f^{2}\right)}$ of the unit allocation.

Allocations for a few representative units are listed in Table 4. With the exceptions of EDI and DES, the limits for the moment variability, $\mu_{\text {Alloc,Stab }}$, are a factor of 5 to 20 lower

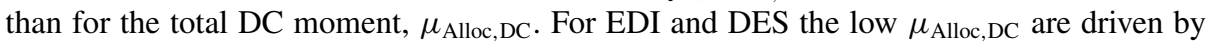
their own requirements since they are the closest units to their own magnetically sensitive volume. The transponder and DES units contain magnets in their designs. In practice, the materials and magnets allocations were lumped together in a root sum square (RSS) sense since they could not be measured separately.

To guide the design of electronics systems, specifically the board layouts and discrete wiring routing, the unit-level allocation for electric currents, $\mu_{\mathrm{Alloc}, I, i}$, was divided between the different voltage services of each unit. For each voltage service, denoted by the index $k$, the engineering teams provided their best estimate for the current, $I_{k}$. For a unit with a total of $N_{S}$ services, the current loop area was allocated by

$$
\begin{aligned}
A_{\text {Limit, }, \mathrm{k}} & =\mu_{\mathrm{Alloc}, I, i} \frac{1}{I_{k} N_{S}} \\
d_{\text {Limit, }, \mathrm{ik}} & =\sqrt{A_{\text {Limit }, \mathrm{ik}}}
\end{aligned}
$$

where $\mu_{\text {Alloc }, I, i}$ is the current moment allocation for the $i$ th unit, and $d_{\text {Limit, ik }}$ is the dimension of a square loop of area $A_{\text {Limit,ik }}$. The root sum square (RSS) approximation is not used for voltage services in the same unit because the board orientations and hence the current loop moment directions are not randomly distributed. The division of allocation among services assumes worst-case addition of moments, that is, that they all have the same sign. Except for the solar arrays, battery, and transponder, for which the entire current counts against the DC stability allocation, the allocations for DC-stability were assigned assuming that the variation of current in each service was $5 \%$ of the total current in each service and the allocations for stability were divided between services using the DC-stability allocation and Eq. (21a) but with $1 / 20$ th of the service current. Table 5 gives the voltage service allocations 
Table 4 Magnetic moment allocations of representative units on the MMS spacecraft

\begin{tabular}{|c|c|c|c|c|c|c|c|}
\hline \multirow[t]{2}{*}{ Unit } & \multirow{2}{*}{$\begin{array}{l}\mu_{\text {Alloc }, \text { Stab }} \\
\left(\mathrm{mA}^{2}\right)\end{array}$} & \multirow{2}{*}{$\begin{array}{l}d B \text { at } 1 \mathrm{~m} \\
(\mathrm{nT})\end{array}$} & \multirow{2}{*}{$\begin{array}{l}\mu_{\text {Alloc, DC }} \\
\left(\mathrm{mA}-\mathrm{m}^{2}\right)\end{array}$} & \multirow{2}{*}{$\begin{array}{l}B \text { at } 1 \mathrm{~m} \\
(\mathrm{nT})\end{array}$} & \multicolumn{3}{|c|}{ DC Allocations $\left(\mathrm{A}-\mathrm{m}^{2}\right)$} \\
\hline & & & & & Materials & Magnets & Currents \\
\hline Battery & 8.78 & 1.76 & 394 & 78.8 & 227 & 227 & 227 \\
\hline Solar array ${ }^{*, a}$ & 7.53 & 1.51 & 435 & 87.1 & 308 & $\mathrm{n} / \mathrm{a}$ & 308 \\
\hline Latch valves ${ }^{*}$ & - & - & 299 & 59.9 & $\mathrm{n} / \mathrm{a}^{\S}$ & 299 & $\mathrm{n} / \mathrm{a}$ \\
\hline Transponder & 16.9 & 3.38 & 74 & 14.7 & 43 & 43 & 43 \\
\hline Power switching unit ${ }^{* *}$ & 8.38 & 1.68 & 406 & 81.3 & 287 & $\mathrm{n} / \mathrm{a}$ & 287 \\
\hline $\mathrm{CIDP}^{\dagger}$ & 11.8 & 2.35 & 106 & 21.2 & 75 & $\mathrm{n} / \mathrm{a}$ & 75 \\
\hline $\mathrm{HPCA}^{\ddagger}$ & 7.4 & 1.47 & 444 & 88.9 & 314 & $\mathrm{n} / \mathrm{a}$ & 314 \\
\hline EDI-GDU ${ }^{*}$ & 15.8 & 3.15 & 10 & 2.1 & 7.3 & $\mathrm{n} / \mathrm{a}$ & 7.3 \\
\hline $\mathrm{DES}^{*}$ & 10.0 & 2.0 & 42 & 8.3 & 24 & 24 & 24 \\
\hline
\end{tabular}

\footnotetext{
* Minimum allocation from all locations of multiple identical units on each MMS spacecraft

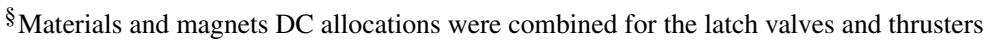

$\dagger$ Combined Instrument Data Processing unit

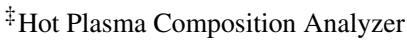

** Formally named the power switching electronics and energy system, PSEES
}

for the CIDP unit, illustrated here because it provides pass-through power service to the remainder of the payload.

The allowed maximum current loop dimensions were refined as follows. First, the minimum between the DC and DC stability $d_{\text {Limit,ik }}$ was used to assign the preliminary $d_{\text {Limit,ik }}$ for each service. The values for $d_{\text {Limit,ik }}$ were ranked for design feasibility in four ranges of design impact: 'none' for $d_{\text {Limit,ik }}>20 \mathrm{~cm}$; 'minimal' for $d_{\text {Limit,ik }}=10$ to $20 \mathrm{~cm}$; 'moderate' for $d_{\text {Limit,ik }}=4$ to $10 \mathrm{~cm}$; and 'severe' for $d_{\text {Limit, ik }}<4 \mathrm{~cm}$. For many voltage services drawing modest currents, $\sim 10 \mathrm{~mA}$ or less, the value for $d_{\text {Limit, ik }}$ from Eq. (21b) was greater than $30 \mathrm{~cm}$, larger than the dimension of most boards on the spacecraft. In these cases, $d_{\text {Limit,ik }}$ was set to $30 \mathrm{~cm}$ and the unused portion of the moment re-assigned to a service with the smallest $d_{\text {Limit,ik }}$. For example, in the CIDP service allocations, the excess moment allocations for the two FEEPS units were re-assigned to the CIDP $3 \mathrm{~V}$ service. Using similar adjustment of allocations between services it was possible to ensure that no service (with the exceptions of the solar arrays and battery) was subject to a severe, $d_{\text {Limit, ik }}<4 \mathrm{~cm}$, current loop constraint. Tables for each unit in the form of Table 5 were disseminated to all of the engineering teams and formed the basis for reviews of all electronics board designs. Expressing the allocations in terms of the $d_{\text {Limit, ik }}$ proved to be particularly accessible to engineers who are generally not accustomed to calculating magnetic moments.

\subsubsection{Low Frequency Magnetics Emissions}

The power spectral noise levels of the fluxgate (analog, AFG, and digital, DFG) and search coil magnetometers are shown in Fig. 30. The lower envelope of the fluxgate and search coil magnetometer sensitivities was taken as the observatory emission limit and the frequency range of 0.02 to $30 \mathrm{~Hz}$ monitored by the magnetics cleanliness effort. The unit-level requirement was set to have a power spectral density one order of magnitude lower than the observatory limit. This reflects the assessment that very few units operate with significant 
Table 5 Example service allocation for the Combined Instrument Data Processing (CIDP) unit

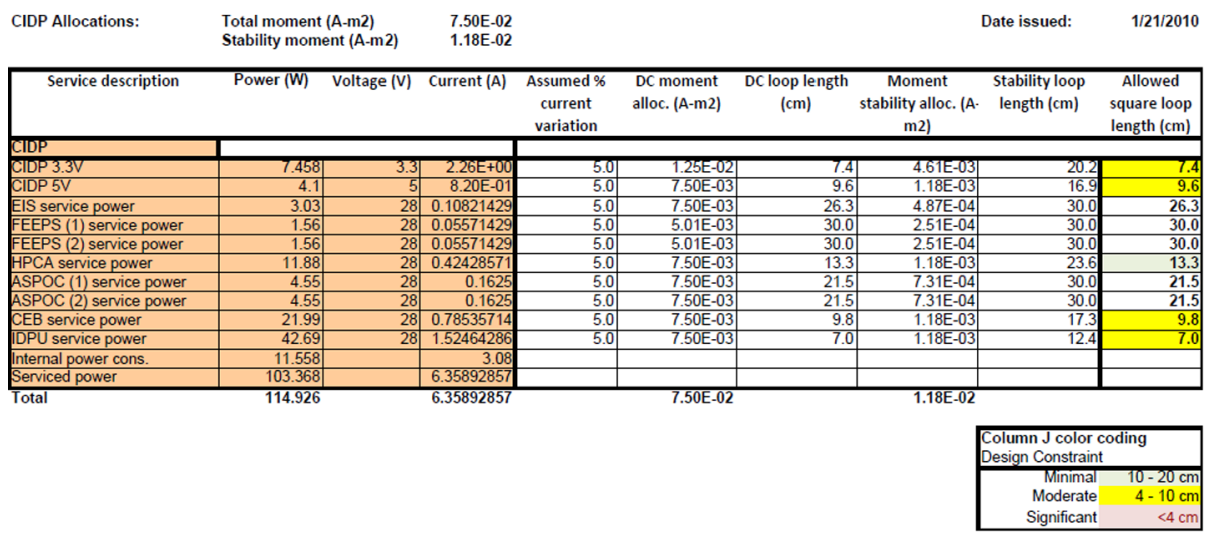

current variations in this frequency range so that overlap at a given frequency from multiple units was considered unlikely. Testing showed that only the HPCA and the Combined Electronics Box (CEB) of the Fields Investigation emitted in the 0.02 to $30 \mathrm{~Hz}$ range, and that their emission frequencies were different.

\subsection{Development Effort and Mitigation Steps}

The magnetics allocations described above provided quantitative guidance in the design, development, and testing of the spacecraft, subsystems, and components. Several items that could be the strongest magnetic sources on the spacecraft were identified early in development and were given particular attention. These included: the magnetically latching propulsion system valves, propulsion system thrusters, solar arrays, spacecraft battery, power switching and distribution unit, transponder, and the CIDP and CEB which service power to other units. Magnetization of permeable materials was also a concern and a program was adopted for materials selection, component screening, degaussing, and handling. The allocations for allowed current loop dimensions were disseminated to the development teams and board layout and wiring designs were reviewed to ensure that current loops were avoided where necessary. In almost all units, some modest adjustment in the preliminary layout design was recommended. Current loops in the harness were minimized by requiring all power wiring to be in power/return twisted pairs. Chassis ground power returns were minimized by requiring a minimum resistance to ground in all electronics units and care was taken to avoid parasitic grounding through signal lines. Testing and requirements for operational heaters were also developed to ensure that the field stability requirements were met.

\subsubsection{Total Field Assessment and Mitigation Steps}

Permeable materials are ubiquitous in electronics components since electromagnets are commonly used for robotic handling of integrated circuit parts. This also implies that they are usually magnetized. Testing with representative components established guidelines for the maximum allowed magnetic field intensity exposure. Most materials required a field of over 20 Gauss to magnetize whereas the soft steel casings of the battery cells magnetized for fields as low as 10 Gauss. A limit of 5 Gauss was identified as a safe threshold below 
which components are unlikely to magnetize. A program of degaussing electrical units was implemented to ensure against residual magnetic moments due to manufacture or handling prior to integration. Handling processes were adopted to ensure that no spacecraft components or sub-systems were re-exposed to potentially magnetizing magnetic fields. Payload electronics boards were screened and degaussed at the board level while spacecraft avionics were degaussed at the unit level.

The greatest challenge in reducing exposure to magnetizing fields turned out to be vibration tables since solenoidal coils producing substantial magnetic fields are part of the vibration driver. There is considerable variation with manufacturer and model in the fields generated by vibration units at the mounting locations of items under test, ranging from only a few Gauss to over 50 Gauss. Vibration tables at the various integration facilities were tested to identify which specific models exceeded the allowed maximum field and alternatives or fixtures were used to ensure that vibration testing did not re-magnetize materials. At the Goddard Space Flight Center integration facility, it turned out to be more cost effective to degauss avionics sub-systems after vibration testing to ensure that components delivered to the spacecraft were demagnetized.

Since the magnetics requirements do not apply during thrusting or deployments, the magnetic fields during actuations are not of concern, but the static moments of units with permanent magnets were considered. Units with strong permanent magnets included the latching propulsion valves, battery relays, and radio-frequency (RF) waveguide switch. The battery relay moments were below the battery DC allocation for magnets. The RF switch magnets exceeded the RF system DC allocation but were below the available observatory allocation at the RF switch location after accounting for the actual allocations used by other units, so this exceedance did not require mitigation. However, the difference in moment in the two positions exceeded the observatory allocation for variable moment and an operational constraint was therefore adopted restricting RF-switch operation to exclude the science region of interest. The propulsion system valves are magnetic latching valves and their moments greatly exceeded their allocations and cancellation magnets were required to reduce their total field to acceptable levels as described in Sect. 5.4 below.

\subsubsection{Power System}

The solar arrays were of particular concern because the MMS spacecraft are designed to spin at $3 \mathrm{rpm}$ about an axis nominally normal to the spacecraft-Sun line. Thus, all of the array panels pass into and out of sunlight with every spacecraft spin. This implies that the total solar array current had to be applied to the field stability allocation. The allowed loop area for each array was therefore less than $4 \mathrm{~cm}$ on a side and avoiding current loops was a major consideration in the array design. This necessitated mesh backwiring, in which the return current flows directly underneath each string of solar cells, and is mounted integrally with each string on the front/outer face of the array. A total of nine strings populate each array. Each string runs the entire length of the array to facilitate the mesh backwiring design. In addition, all solar array wiring, on the backside of each panel, uses twisted pairs to carry the power/return of each string. The only break-outs from the twisted pairs occur at the solar array connectors and at the by-pass diode pads located on the backs of the arrays. Qualification model testing revealed that the wire routing on the by-pass diode pads needed to route the power/return lines with alternating polarities between strings to meet the magnetics emission stability requirement. This slight modification was implemented and its effectiveness verified in test (see Sect. 5.4 .3 below).

The battery current varies only when the state of charge changes. The largest state transitions occur at entry and exit from Earth eclipse, in which the battery transitions between 
maximum discharge and maximum charge current. The mission does not require compliance with the magnetics cleanliness requirements at entry and exit from Earth eclipse, but the battery recharge time will overlap with science observations so the field stability requirement was applied to the total battery charge current. The banks of battery cells were configured in opposing ' $\mathrm{T}$ ' sections and the power/return lines were designed to be as adjacent as practically possible.

Although the requirement to minimize current loops was included in the battery specifications, the wiring between the cell banks and the harness connector was not called out in particular as a design constraint and this aspect of the design did not come up in the battery design reviews. Initial testing of the qualification battery revealed a current loop with $\sim 15 \mathrm{~cm}$ diameter. This turned out to be due to the wiring in the battery between the cell banks and the battery relays prior to the output harness connector. Because of schedule constraints, it was not possible at that time to re-design the wiring in the battery. Instead, a compensation loop was added to the harness within $30 \mathrm{~cm}$ of the battery. Custom testing of each battery was done to establish the precise direction and magnitude of the current loop moment and the harness compensation loop was sized and oriented differently for each battery. The combined battery and cancellation loop were then tested to verify both that the cancellation loop polarity was opposite the battery loop and that the resulting dipole field was cancelled to within $10 \%$ to meet the stability requirement with a change in current up to 10 Amperes.

All of the spacecraft power is managed and distributed by the power switching electronics and energy system (PSEES). Particular attention was therefore paid to the board layout designs, discrete wiring routing, fuse wiring, and backplane design of the PSEES. Early prototype and qualification units of the PSEES were tested to ensure that nothing had been overlooked. These tests and subsequent tests of the engineering and flight units confirmed that the PSEES complied with all of its allocations with generous margins.

Finally, the harness was specified to carry all power via twisted pairs of power and return lines to minimize any magnetic fields from the distribution wiring. The fully redundant $\mathrm{A}$ and $\mathrm{B}$ sides of the PSEES and other spacecraft avionics were designed so that the power grounds between the A and B units were isolated. Many of the payload electronics units however used semi-redundant designs because the science measurements achieved some redundancy across instruments. This had the unfortunate consequence that the A and B side electronics in some instrument units shared a common power ground which had the impact of distributing the power return between the A and B units and their harnesses while the power remained only on either A or B. The PSEES was designed to switch only the power side of loads so that both $\mathrm{A}$ and $\mathrm{B}$ grounds remained active. To eliminate spurious A-to-B ground sharing, a cross-switching box (XBOX) was developed to switch both the power and grounds of A and B sides to the payload. This ensured that the shared instrument grounds in payload units did not allow power return current to flow through the unused side. The XBOX was mounted near the PSEES and was assigned a magnetics allocation from reserves and subjected to the same testing as all other electronics units.

\subsubsection{Distributed Spacecraft Currents}

Currents to heaters and inadvertent grounds to the spacecraft chassis were also examined in detail to establish allowed limits and guide the designs to ensure that these distributed currents did violate the magnetics requirements of the spacecraft. The propulsion system and nearly all electronics sub-systems employ thermostatically controlled heaters to ensure acceptable temperature limits for operations and also for survival in the event of restricted 
operations associated with anomalies. Because the heaters operate thermostatically without commanding, telemetry records of heater operation do not identify which heaters draw current at any given time. All thermostatically controlled heaters were identified and itemized by power and current draw. Allocations were assigned to the collection of heaters for maximum total and variable emitted fields.

The wiring to all heaters was required to be in twisted power/return pairs breaking out only for connectors and thermostats. In addition, all heating elements were required to be non-inductive, which typically implied using two-layer heater elements in which the current flowed in opposite directions in the layers. Samples of each heater element to be used were tested to ensure that the selected units were in fact non-inductive. The field stability requirements determined the maximum allowed uncompensated loop area (at the thermostats) for each heater circuit assuming realistic time constants for thermostatic switching and an RSS estimation of the total moment from the heater circuits. Allowed areas of several $\mathrm{cm}^{2}$ at each thermostat were acceptable so that configuring wiring at the thermostats to be selfcancelling was not necessary provided that unnecessary excursions around the thermostats were avoided.

The heaters also contribute to the low frequency magnetic emissions when the thermostats switch on and off, resulting in step changes in the low level magnetic field their small but finite current loops generate. We also considered the fact that mechanical thermostats do not close in a single motion but the contact point actually bounces mechanically a very small distance resulting in repeated close-open-close transitions. The transition from closed to open was verified to be a single switch-off as expected. The actual current transitions of representative thermostats were characterized for the statistical distribution of the number and duration of bounces. The entire bounce sequence takes less than $5 \mathrm{~ms}$ with 2 to 7 bounces. Simulated power spectra of the emissions demonstrated that the corresponding emissions using the allocated heater loop areas are below the magnetics emission requirements.

If ground returns occur through the chassis rather than the power return line, not only is the purpose of the twisted pair cabling ineffective but the resulting current moments have dimensions comparable to the spacecraft radius. Particular care was therefore taken to ensure that a minimum resistance to chassis ground was imposed in all electronics designs. Signal interfaces were identified as one area in which unintended grounds were likely, and impedance limits were included in these designs as well. The PSEES units were designed with chassis current monitors to provide a telemetry point of chassis currents. Preliminary tests were conducted with prototype harness and available electronics units to develop techniques for independently verifying that there were no magnetic field signatures indicative of chassis currents. The final tests developed for this purpose are described in Sect. 5.5.1 below and confirmed that any chassis currents are less than $1 \mathrm{~mA}$, consistent with the designs.

\subsection{Unit-level Testing Program}

Compliance with the allocations and success of mitigation steps were verified with a comprehensive testing program. Because of the large number of units and institutions involved, a four sets of magnetic field test equipment were assembled and distributed to key parties: Goddard Space Flight Center (GSFC) where the spacecraft were developed and integrated; Southwest Research Institute (SWRI) where many of the instruments were delivered enroute to GSFC; the University of New Hampshire (UNH) where the Fields instrument suite was delivered and tested; and JHU/APL where the magnetics cleanliness effort was headed. The test set at JHU/APL was used primarily for testing at vendor locations although the 
GSFC test set was occasionally also used for remote site testing. The test equipment was developed by GSFC and consisted of a commercial fluxgate magnetometer with $\sim 1 \mathrm{nT}$ noise, a 20-bit multiple channel A/D converter, a USB interface unit, and a dedicated laptop computer for data acquisition control and real-time display. In addition, two units supporting four fluxgate test magnetometers were constructed to support observatory-level testing (Sect. 5.5 below) although the multiple magnetometer system also proved useful in some unit level tests.

\subsubsection{Electronics Units Testing}

Compliance of all electronics units with magnetics allocations was verified either through testing conducted by MMS team members or in a few exceptional cases by the vendors. The vast majority of these tests were conducted using the field-test fluxgate system in normal laboratory environments. The testing consisted of three parts: unpowered DC or fixed moment measurement; powered moment measurement, including both the steady state (DC) and variable (DC stability) moments; and low frequency emissions measurement, or low frequency AC. The tests were designed to provide upper limit estimates of unit magnetic moments to either demonstrate that the moments were lower than the allocation or identify articles that required more extensive testing. The tests were designed to allow two stages of analysis, preliminary worst-case estimates, which in most cases gave results with sufficient margin that no further analysis was needed, and slightly more rigorous analysis that relaxed some worst-case, simplifying assumptions.

Practical considerations influenced the design of the testing. The number of units to test exceeded 200 so it was essential to keep the testing simple and suitable for a normal laboratory environment. To allow measurements in a standard environment, measurements were made within 0.5 meters to take advantage of the $1 / d^{3}$ dependence of the dipole field. This afforded a factor of greater than 1000 increased signal to noise relative to measurements conducted at the fluxgate sensors deployed distances. In this way, even with the moderate performance test fluxgate system, the field-test measurements were capable of detecting moments below $1 \%$ of the allocations. For most units the complete test procedure was used only for the engineering model and first flight model and an abbreviated test procedure, adjusted per the full test results, was used for the remaining unit.

For some units carrying very low currents, the initial tests revealed such a low level of magnetic signals that subsequent testing was either waived altogether or conducted on only a convenient subset of units. Such units included the digital sun sensors, GPS low noise amplifiers, GPS antennas, and propulsion system pressure transducers. In the case of the star camera heads and associated processing unit, which had also flown on the high precision magnetic measurement missions Oersted and CHAMP, the vendor documentation and heritage were sufficient to certify compliance.

The unpowered test procedure consisted of moving the unit from a distance of at least 1 meter in to $\sim 20 \mathrm{~cm}$ from the test magnetometer and repeating this out-in-out displacement three times. This procedure was repeated with all six faces of the unit oriented toward the test magnetometer as illustrated in Fig. 32. The figure also shows results of these measurements for the one of the HPCA units. For some units it was impractical to manipulate the unit with the top and bottom faces toward the magnetometer sensor, so four orientations were used. The relative displacement provides a factor of $\sim 100$ change in the magnetic field due to the unit at the test magnetometer so that the residual field at the 1 meter distance is negligible. For the HPCA results shown, the variation between the three repetitions of each out-in-out displacement for each position indicate that the repeatability gives results accurate to $20 \%$ 

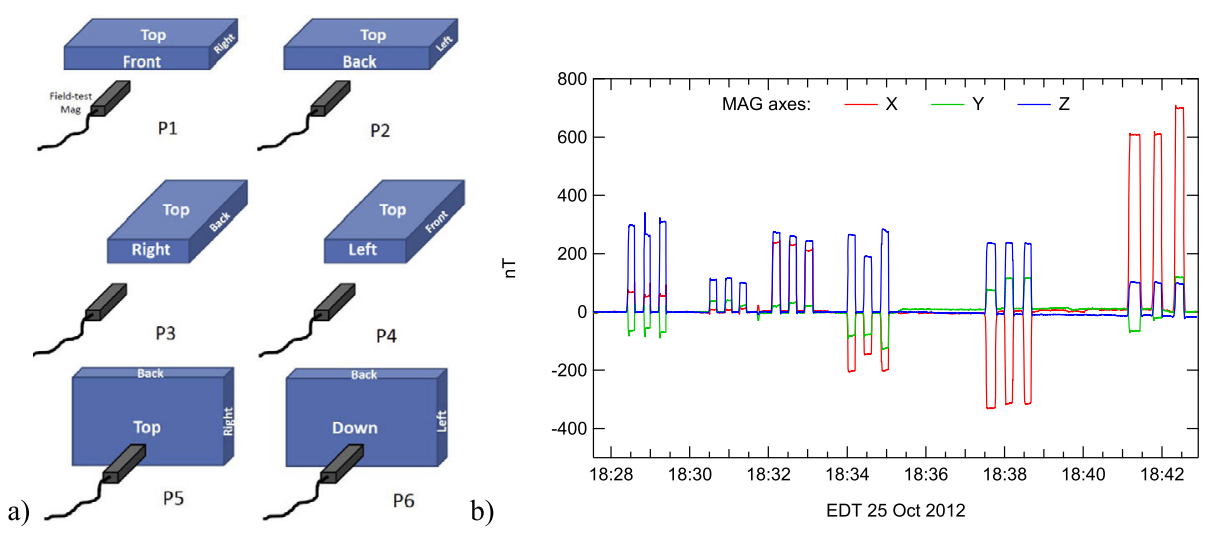

Fig. 32 Illustration of the six-position test for unpowered magnetic moment assessment (a) and results for an HPCA unit (b). For all units at least positions P1 through P4 were used and for most units P5 and P6 were also used. For each position, the article under test was displaced from a distance greater than 1 meter toward the test magnetometer to within $20 \mathrm{~cm}$. On the right, the $X$-axis (red) was directed away from the unit, the $Y$-axis (green) is to the right looking toward the unit, and the $Z$-axis (blue) is upward. The directions $x, y$, and $z$ here refer to the coordinate system of the test item

on average. It is also evident that the signals are more than an order of magnitude greater than variations in the ambient magnetic field in the facility which was a conventional space hardware development laboratory. The moment estimated from these data is $15 \%$ of the materials moment allocation confirming that testing in normal laboratory settings provided ample signal to noise relative to the requirements.

Test data were analyzed to estimate the magnetic moment of the unit as follows. For a point dipole, the moment, $\mathbf{m}$, and magnetic field, $\mathbf{B}$, at a position, $\mathbf{r}$, are related by

$$
\mathbf{B}=\frac{\mu_{0}}{4 \pi}\left(3 \frac{(\mathbf{m} \cdot \mathbf{r}) \mathbf{r}}{r^{5}}-\frac{\mathbf{m}}{r^{3}}\right)
$$

If we let the $X$-axis be along $\mathbf{r}$, this immediately gives

$$
m_{X}=\frac{B_{X} x^{3}}{200}, \quad m_{Y, Z}=-\frac{B_{Y, Z} x^{3}}{100}
$$

where $\mathbf{B}$ is in $\mathrm{nT}, \mathbf{m}$ is in A- $\mathrm{m}^{2}$, and $x$ is in meters and assumed positive. With the test data acquired as described, the point-dipole approximation is surprisingly useful for the moment estimates. First, one can readily assess whether the moment is predominantly permanent or induced by the ambient Earth magnetic field. The reversal in polarity of the $X$ component between the position pairs P3/P4 and P5/P6 indicate that the moment is predominantly a permanent moment rather than induced, since if it were induced, it would not reverse with the unit orientation. Second, with (23) it is evident that this HPCA unit's dominant moment is directed vertically downward accounting for the approximately factor of two difference between the $Z$-axis field in positions P1 through P4 and the $X$-axis field in P5 and P6, as well as the signs of the $X$ and $Z$ components. There is a transverse component to the moment as well, evident in P3/P4. Third, the difference in signal amplitudes between P1 and P2, and between P5 and P6 imply that the moment is not centered in the unit but displaced toward the front and bottom from the unit center. Given the distances used and dimensions of the unit one can estimate the moment location from these data. For a distance to the test 

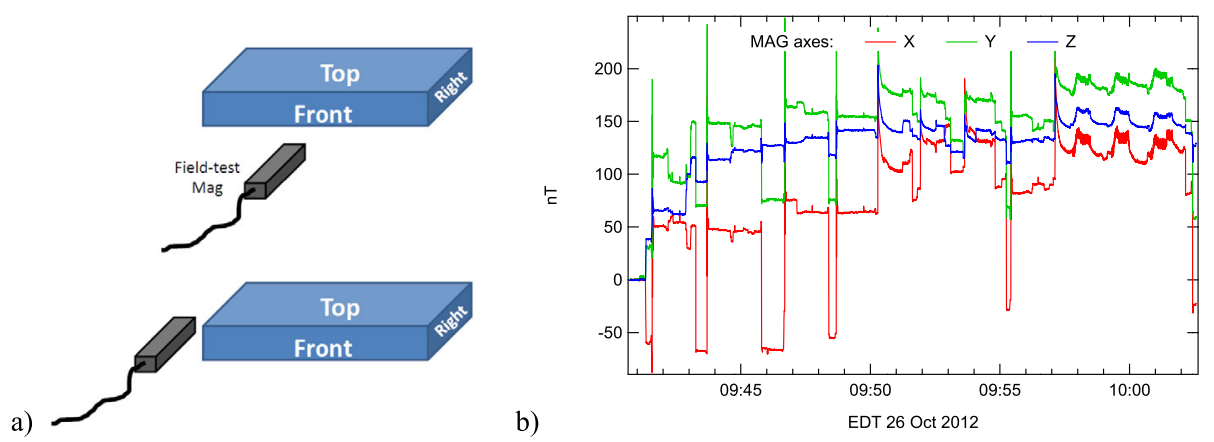

Fig. 33 Illustration of the two-position test for powered magnetic moment assessment (a) and results for an HPCA unit for position P7 (b). On the right, the $X$-axis (red) was directed away from the unit, the $Y$-axis (green) is to the right looking toward the unit, and the $Z$-axis (blue) is upward. The off to baseline-on cycles were done from 09:41 to 09:50 while the baseline-on to science mode cycles were done from 09:50 to 09:55 and the science to fast survey cycles were done from 09:57 to 10:02

magnetometer equal to half the unit dimension, the difference in observed field for a moment at the near and far faces of the unit is a factor of 27 so the displacement of the moment from center is fairly modest in this case.

In most cases, the data from each of the orientations was used to estimate a moment magnitude from (23) assuming a centered moment and the largest of these moment estimates was compared to the allocation. No further analysis was done if this worst-case estimate was within the allocation. This was sufficient for all but a few units. If necessary, the moment estimate was refined by allowing the moment to be displaced so that the magnitudes measured on opposite faces agreed. In addition, the field component directed toward the unit was used to separate the permanent and induced moment signature. An outage was logged only if the least conservative estimates of the moment exceeded the allocation. The only electronics units exhibiting outages were the CIDP, SDP, and EDI-GDU and the violations were small enough to accommodate by re-assigning unused allocations of other units. The unpowered test was waived for units if they did not contain magnets, used less than a few percent of their allocation in initial unit tests of the engineering model or first flight unit, and were degaussed at the unit level.

The powered tests were designed to determine the total moment due to currents, the moment stability during science operations, and the low frequency magnetic emissions in a single procedure. For the engineering unit and first flight unit, the test magnetometer was positioned in front of two faces of the unit, denoted P7 and P8 as shown in Fig. 33a. The unit was not moved since this is typically not possible due to cabling. For each position, P7 and P8, two distances were used, typically $\sim 10 \mathrm{~cm}$ and $\sim 20 \mathrm{~cm}$. For each position and distance, the following sequence of states was exercised: off to baseline-on, three times; baseline-on to operational, three times; operational to fast survey mode, three times. For most spacecraft avionics there is no distinction between operational and fast survey so only unique states were used. For the transponder the transitions between transmitter on and off were used. Figure 33b shows the results of one such sequence for HPCA using P7 at the closer distance. The step changes at transitions were averaged over each set of cycles to discriminate against variations in the ambient field. The signals from the unit were clearly resolved yet the derived moment due to currents was only $4 \%$ of the HPCA allocation reflecting the generous sensitivity of the test relative to the requirements. 

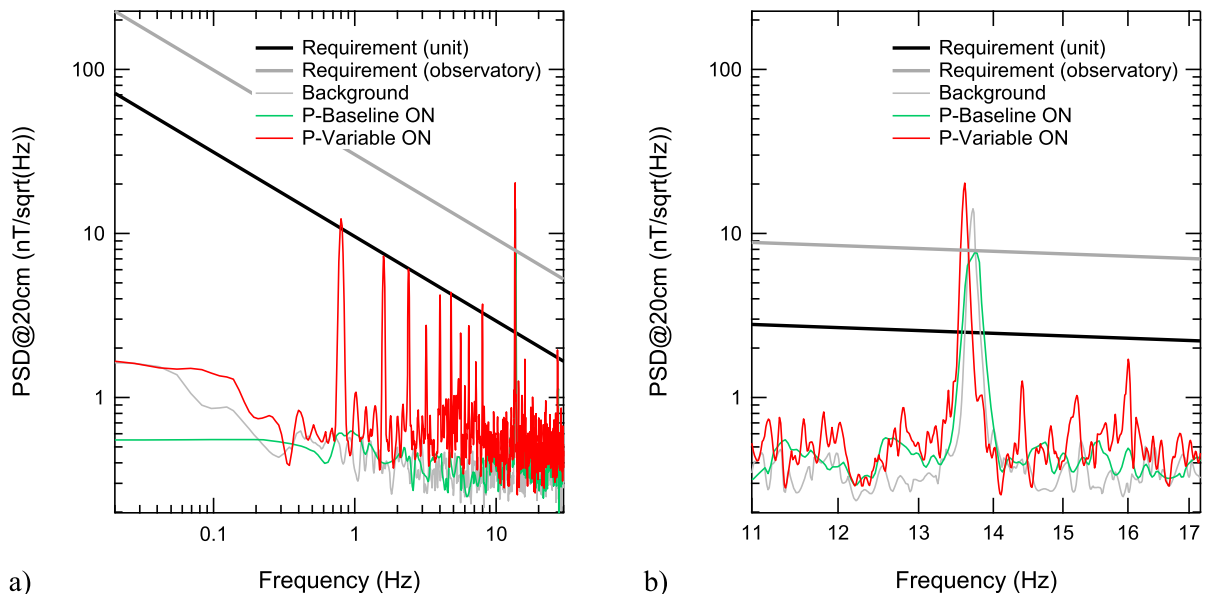

Fig. 34 Amplitude spectra for the low frequency magnetics test for an HPCA unit. Spectra show the trace of the spectral matrix. The gray trace is the ambient conditions, the green trace is powered on but not in science mode, and the red trace is with the unit in fast survey mode. The left panel (a) shows the full frequency range from 0.02 to $30 \mathrm{~Hz}$ and the right panel is an expanded view near $14 \mathrm{~Hz}$. The observatory requirement is the thick gray line and the unit-level requirement is in black

To measure the field stability, data were recorded for a five minute dwell in the fast survey mode and the variance in the field used to derive an upper limit emission signal. For most units, the ambient background contributed more to the field variability than the unit but ambient variations were typically less than a few nT. For HPCA the test yielded an upper limit estimate for the moment variation that was only $7 \%$ of the DC-stability allocation.

Low frequency magnetic emissions were measured by sampling the test magnetometer at $60 \mathrm{samples} / \mathrm{s}$ and recording data for five minutes with the unit in three states: powered off; operating in normal science mode; and operating in fast survey mode. Data recorded with the power off provide background levels and departures from this background provide confirmation that signals are from the unit. For most units there was no significant distinction between background and unit on modes. The amplitude spectra for HPCA for this test are shown in Fig. 34. All spectral amplitudes were normalized to a common distance of $20 \mathrm{~cm}$ using $1 / d^{3}$ scaling from the measurement position relative to the unit center. The observatory limit corresponds to the curve of Fig. 34 scaled from $4 \mathrm{~m}$, the distance of the SCM when deployed, to $20 \mathrm{~cm}$, the distance in the test. The thick gray line is the unit spectral amplitude limit, a factor of $\sqrt{10}$ lower than the observatory limit. The ambient amplitude spectrum shows that the test was sensitive and could resolve signals from the unit well below the requirement. There is one peak at 13 to $14 \mathrm{~Hz}$ which extends above the observatory limit and the expanded view shows that this is environmental. A series of peaks at $0.8 \mathrm{~Hz}$ and harmonics are generated by HPCA in fast survey mode and reach the unit-level requirement. These emissions were present in all HPCA units and the proximity of the amplitude to the requirement prompted a set of additional measurements at a range of distances from HPCA in the direction of the nearest victim to low frequency emission. These tests established the source location within HPCA more precisely and confirmed that HPCA complies with the unit level emission allocation. Observatory-level tests (Sect. 5.5.2 below) confirmed this conclusion.

Complete power sequence of tests was performed at both P7 and P8 and at two distances of the test magnetometer relative to the unit. This full test was done on the engineering 
model and the first flight model, but subsequent tests used an abbreviated test in which only the position and nearest distance giving the largest signals were used, reducing the length of the powered portion of the test by $75 \%$. The abbreviated tests demonstrated similarity in family with the unit that was tested more extensively.

\subsubsection{Propulsion System Testing}

The propulsion system includes a number of components of magnetics concern. The major issue was the magnetic latching valves. In addition to the valves, the thrusters are magnetically actuated and it was necessary to use stainless steel for the propulsion lines whose magnetization properties were not known. The propulsion line samples were found to acquire negligible magnetization retiring this concern. A subset of the flight thrusters, representing at least two of each type of thrusters, were also tested for residual static fields using the unpowered test procedure described above, and found to be well below their allocations indicating that the permeable cores of the magnetic actuators do not retain significant magnetization.

The magnets of the latch valves had moments which exceeded their total fixed moment allocations by more than a factor of ten. Moreover, four latch valves were used on each spacecraft so that they presented a significant risk to the DC magnetics requirements. The excess was identified early using a qualification valve while the propulsion system layout was in its early design phase. The latch valve moments of all 16 flight valves were then measured precisely at the JHU/APL magnetics facility. The propulsion latching valves, in the position used during science observations, each possessed moments of $3.86 \mathrm{~A}-\mathrm{m}^{2}$ on average which greatly exceeds the allocation of 0.299 A-m ${ }^{2}$ (cf. Table 4). The moments of the valves were highly consistent varying by less than $2 \%$ of the average moment magnitude in any component. Figure 35 illustrates a latch valve, the average magnetic moment, and the cancellation magnet location and moment used to mitigate the valve field. The largest component of the moment was vertical $(\mathrm{V})$ relative to the mounting plane of the valve. The $\mathrm{V}$-component moment was 6 times greater than the moment in the horizontal $(\mathrm{H})$ direction aligned with the direction of flow through the valve. The transverse moment was less than $1 \%$ of the vertical moment in every case. The L-shaped bracket below the valve (in this view) was included in the design to accommodate a vertical cancellation magnet. The vertical moments of valves were identical across all of the valves to within $1 \%$ and they were mounted in adjacent locations and in opposing vertical orientations such that vertical cancellation was not required. The single cancellation magnet cancelled the H-component moment of both latching valves mounted in adjacent locations. The cancellation magnets were manufactured from sections of $1 / 4^{\prime \prime} \times 1 / 4^{\prime \prime} \times 6^{\prime \prime}$ Alnico magnets whose lengths were chosen to yield a moment equal to the sum of the H-component moments of each pair of adjacent valves. The cancellation magnet was mounted with its moment opposite to that of the valve $\mathrm{H}$-component moment. Two cancellation magnets were used on each spacecraft and the mounting polarity was verified on all flight units using a hand-held Gaussmeter.

\subsubsection{Power System Testing}

The key parts of the power system tested during development were the PSEES, solar arrays, and battery. The PSEES was tested extensively for both static moments from materials and magnetic signals due to currents. The test procedure for the materials moment was the same as other units described above but the powered test was customized for PSEES and used one of the 4-magnetometer test-sets so that sensors could be placed around the PSEES to sense 


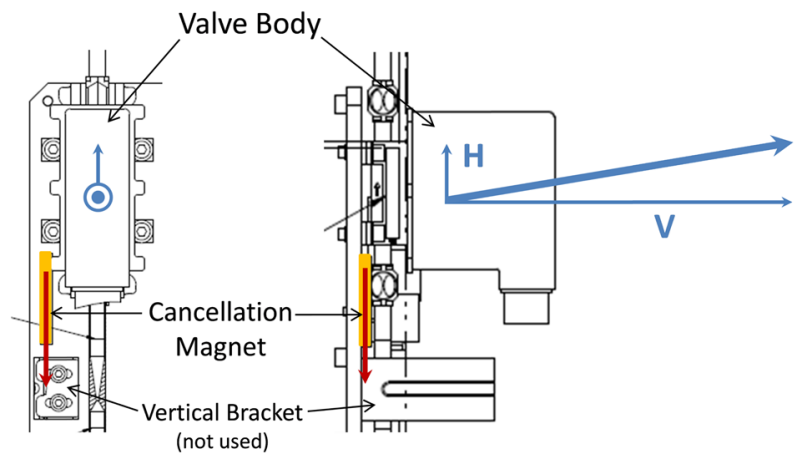

Fig. 35 Magnetic latching propulsion valve showing the magnetic moment of the valve (blue arrows) with the vertical (V) and horizontal $(\mathrm{H})$ components shown to scale together with the cancellation magnet (yellow) and its moment (red arrow). The transverse moment (to the right in left panel and into the page on the right) was less than $1 \%$ of the total moment and is not illustrated. Valves were mounted in pairs with opposing vertical directions but the same horizontal direction. The cancellation magnet moment was designed to equal the sum of the H-component moments of the pair of adjacent valves

the field in multiple locations simultaneously. The distribution of magnetometers and power test data for the PSEES SN2 is shown in Fig. 36. For this test the PSEES was turned on and then simulated (i.e. resistive) loads were switched on and off corresponding to progressively powering on the spacecraft avionics, solar array and battery, simulations of different power states, and then the payload was powered on. By 15:18 EDT the payload was fully powered.

The signals were largest in MAG 2 and MAG 3 and the field was horizontal directed normal to the electronics board planes at MAG 2 but vertical at MAG 3, consistent with a moment directed from MAG 2 to the unit and to the left of MAG 3. MAG 1 and MAG 4 were approximately $30 \mathrm{~cm}$ from the moment and at these locations the maximum signal was already only about $20 \mathrm{nT}$. The corresponding moment was estimated to be $23 \mathrm{~mA}-\mathrm{m}^{2}$ consuming only $8 \%$ of its allocation. The DC-stability moment was $3.7 \mathrm{~mA}-\mathrm{m}^{2}$ consuming slightly less than half of this allocation. All of the PSEES units had similar margins.

Testing for the solar arrays was customized to search for magnetic emissions due to current loops. Tests at the vendor with the qualification unit used reverse biased current through the strings while measuring the magnetic field at multiple locations with the 4-magnetometer test set. The mesh backwiring and twisted pair harnessing proved to be completely successful as no magnetic emissions were detectable even as close as $10 \mathrm{~cm}$. However, the diode pad routing, shown in Fig. 37a proved to generate a net moment. The eight solar panels are configured in two sets of four with the strings of the four panels wired in parallel. Each string is served by a redundant parallel twisted power/return pair with two blocking diodes on each panel for each string to prevent a failure in a string from shorting out all of the strings in the set. The current from all nine sets of parallel strings are summed in the PSEES. The solar array pad wiring is shown schematically in Fig. $37 \mathrm{~b}$ and the problem with the qualification unit was that the break-out from twisted pair cabling, required at the diode pads, created current loop moments from each diode pad that were in the same sense. The recommended change shown in Fig. 37c was to route the returns of each redundant pair on opposite sides of the diode pads leading to approximate cancellation between the loops.

Because the only way to test the arrays was to reverse bias the strings, which the blocking diodes prevent, wire jumpers at each diode were used to pass current through the arrays with a power supply. While this test confirmed that mesh backwiring and twisted pair cabling were free of measureable magnetic fields, the wire jumpers had areas comparable to or 

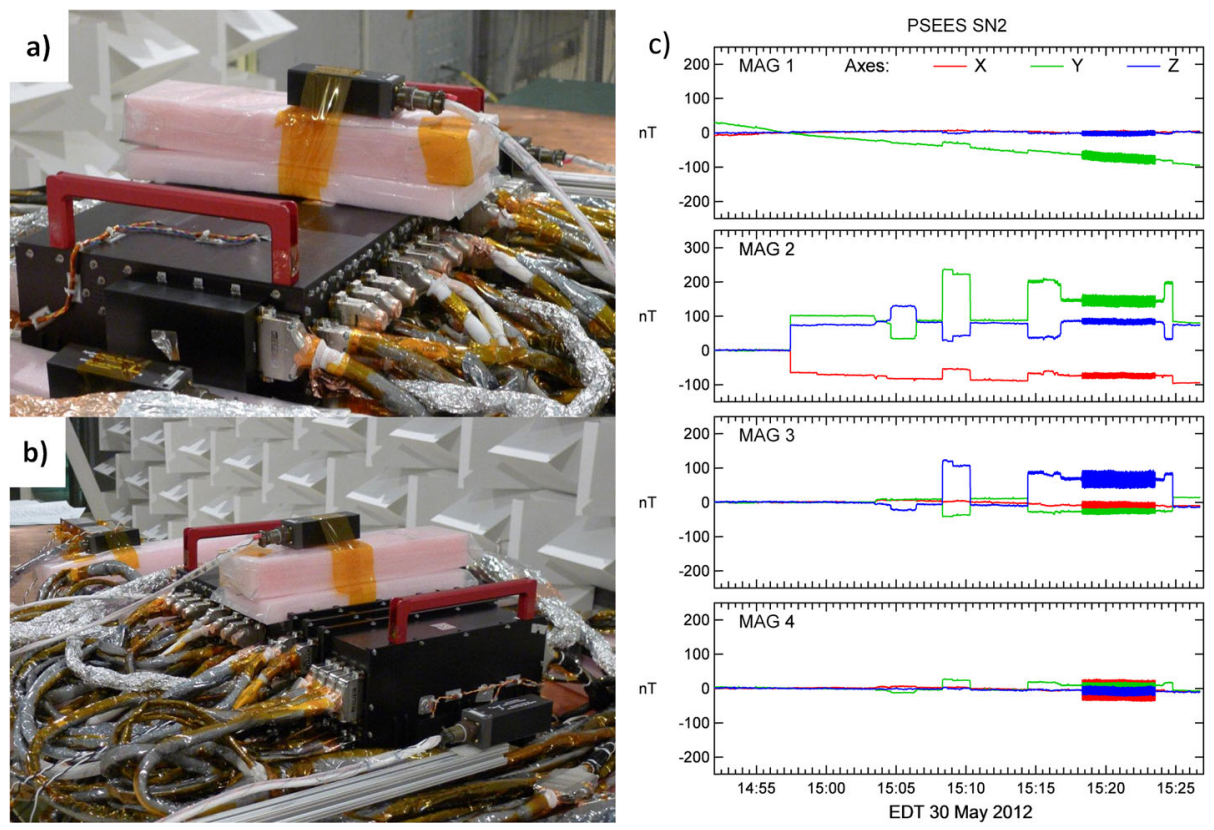

Fig. 36 Magnetometer positions (a) and (b) for the PSEES SN2 powered magnetics test. The magnetometers were arranged in order, MAG 1, 2, 3, and 4, from far to near in (b). MAG 2 is just to the left of the unit as viewed facing the connector plane and MAG 3 was positioned above the unit. Data for the PSEES side 'A' is shown in (c) and this unit is closest to MAG 2. For all four test magnetometers the $X$-axis is directed positive outward from the connector plane, the $Y$-axis is to the right as seen viewing the connector plane and the $Z$-axis is upward. The interval from 15:18 to 15:23 where the magnetometer traces appear thicker is the 5 -minute interval of sampling at 60/s for the low frequency $\mathrm{AC}$ portion of the test during which the signal is dominated by ambient noise
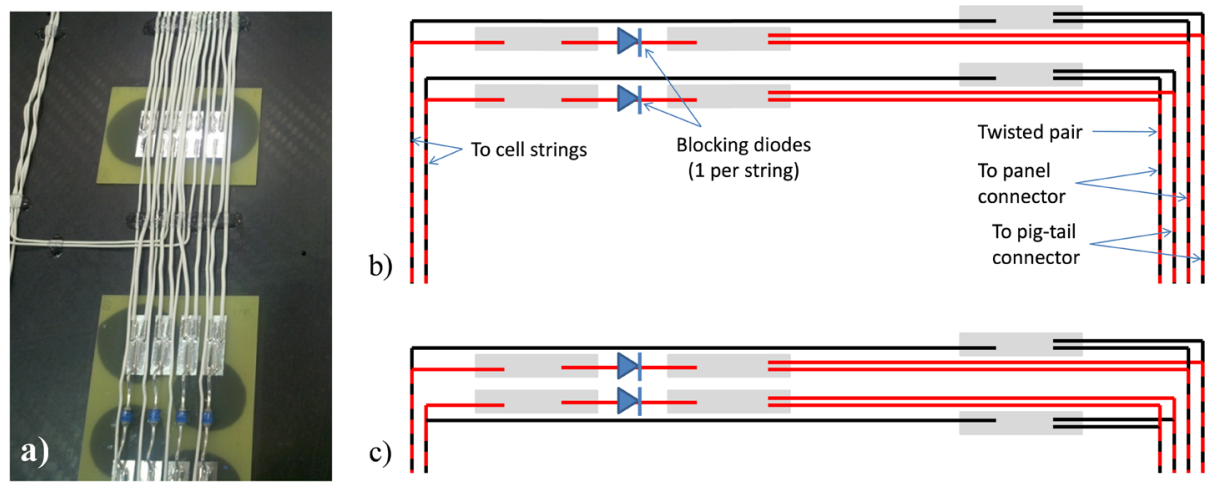

Fig. 37 Solar array blocking diode pads as implemented on the qualification panel, (a); schematic showing the wiring routing for the qualification panel, (b); and revised routing for the flight panels, (c). The lines in (a) to the left of the diode pad area are signal lines used by the power system to monitor voltages that carry negligible current and are neglected in the magnetic field modeling of the panels. In (b) and (c), red traces show the power lines and black traces show the return lines 
Fig. 38 Finite element calculation results of magnetic field magnitude from MMS solar array qualification model including the inadvertent net current loops at the blocking diode pads evaluated at $0.1 \mathrm{~cm}$ (top) and $1 \mathrm{~m}$ (bottom) from the solar array back surface.

Counter-routing the return lines at the diode pads reduces these fields to less than $10 \%$ of these estimates

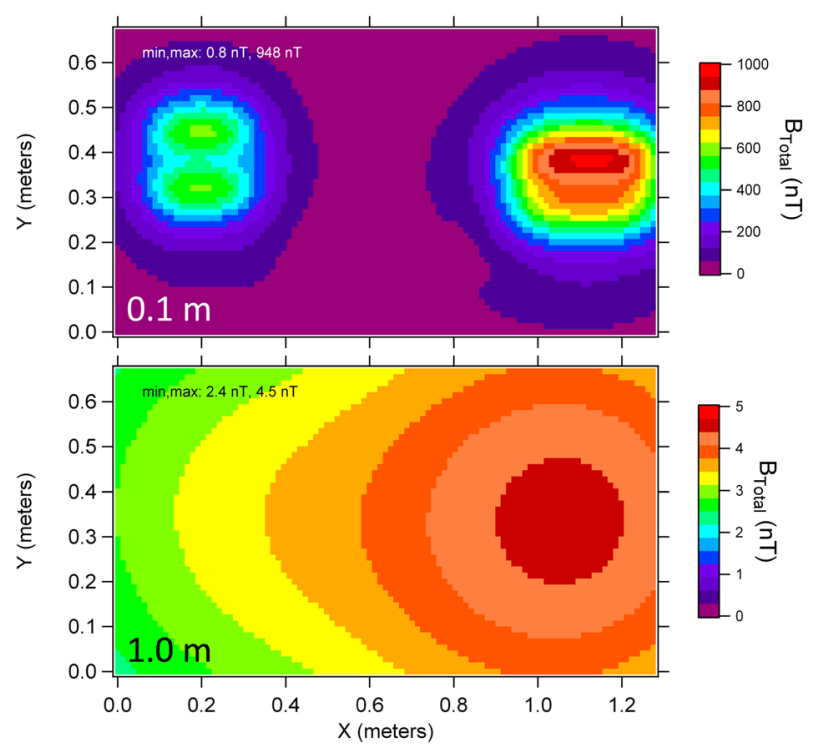

larger than the loops at the diode pads so the field due to these loops had to be quantified by calculation. A finite element model of the current loops in the array wiring, including current imbalances between the redundant parallel wiring for each string, was used for this purpose assuming the total current summed from all four strings. Results of this model at $0.1 \mathrm{~m}$ and $1.0 \mathrm{~m}$ above the panel are shown in Fig. 38 for the qualification panel using the actual wiring layout and assuming $3 \%$ imbalance between the redundant twisted pair cabling for each string. The results at $0.1 \mathrm{~m}$ are consistent with the test measurements, though because of the diode jumpers quantitative comparison was not meaningful. The results at $1.0 \mathrm{~m}$ exceed the DC-stability allocation for the arrays by up to a factor of 3 (cf. Table 3). Implementing the modification of Fig. $37 \mathrm{c}$ to even $20 \%$ cancellation, readily confirmed by visual inspection, brought the arrays under their DC-stability allocation.

Confirmation of the final solar array implementation was obtained with a fight array under illumination from high intensity lights and with a power supply providing the total current through the array wiring and connectors equivalent to all four parallel arrays. The magnetometers of the 4-magnetometer test system were positioned as follows: MAG 1 over the smaller set of diode pads, MAG 2 over an area of the panel without cabling, MAG 3 over the pig-tail panel connector, MAG 4 over the larger set of diode pads. The results are shown in Fig. 39 for an array current of $580 \mathrm{~mA}$ during the flash test and a parallel current of $4.5 \mathrm{~A}$ during the total current test with the power supply. The actual array current is $7.2 \mathrm{~A}$ so the signals during the flash test were inflated by a factor of 12 , and those of the cable test by a factor of 1.4. These estimates were more than an order of magnitude lower than those of Fig. 38 confirming that flight arrays are within their DC-stability allocation.

As mentioned above, the battery cells were configured to ensure that the current paths in the battery pack produced minimal magnetic field. However, the bundles of power and return lines gathered at the outputs of the cell pack were routed separately to accommodate the battery relay switches. Initial testing of the qualification battery revealed a moment of $308 \mathrm{~mA}-\mathrm{m}^{2}$ at 15 A current, which would exceed the DC moment allocation for currents by $35 \%$. Of greater concern however is that the battery current may change by as much as $10 \mathrm{~A}$ in recharging during science observations, but the DC-stability allocation was met 

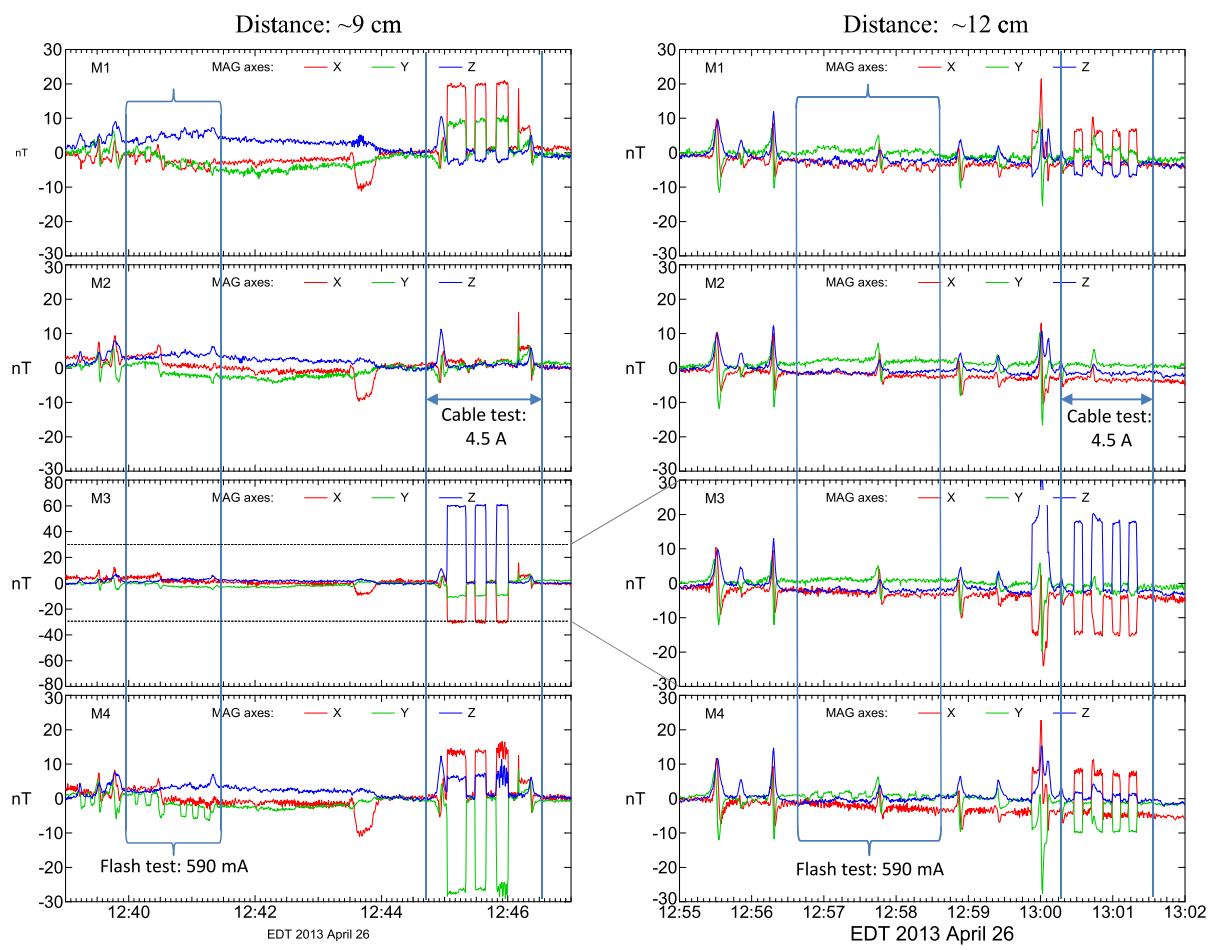

Fig. 39 Solar array flash and panel cable wiring test data showing results from four positions within $9 \mathrm{~cm}$ and $12 \mathrm{~cm}$ of the panel rear surface on the left and right, respectively. Illumination of the array cells with high intensity halogen lamps produced $590 \mathrm{~mA}$ total array current, $\sim 63 \mathrm{~mA}$ per string, and resulted in maximum signals of less than $\sim 5 \mathrm{nT}$ at a distance of $9 \mathrm{~cm}$ evident only near the bypass diode wiring pads, corresponding of signals less than $60 \mathrm{nT}$ under full current. The connector test used a current of $4.5 \mathrm{~A}$ and showed detectable magnetic fields near the connector (M3) and the diode pad areas where the parallel connections are made. The magnetic signals under full current, 7.4 A, are therefore less than $100 \mathrm{nT}$ within $9 \mathrm{~cm}$ of the arrays, well within the array requirements

with a current of only $0.43 \mathrm{~A}$, corresponding to an excess of more than a factor of 20 at $10 \mathrm{~A}$. The addition of a current loop in the harness near the battery was less costly than a redesign and refabrication of the battery wiring associated with the relays. This required careful magnetics calibration of each battery and design of a loop and fixture to hold it. Figure 36 shows the configuration of four test magnetometers around the end of the battery where the relay wiring is located (a) and the harness loop customized for this battery (b). The moment calibration measurements used the sensor configuration of Fig. 40a at two distances from the battery and yields results for the vector moment accurate to a few percent. The harness loop as viewed in Fig. 40b routes the power lines, solid white, in a counterclockwise sense and the return lines, white and blue, clockwise to yield a net magnetic moment predominantly downward with current loop effective area that is twice the physical area of the loop.

Two unit-level tests were performed to ensure that the harness was corrected installed and of the right size. The first test was a polarity measurement to check that the harness loops was oriented right side up. This is done with two test magnetometers one positioned above the battery loop and the other above the harness loop within about 15 to $20 \mathrm{~cm}$ above the loops. A battery current of $\sim 10 \mathrm{~A}$, either charge or discharge was applied to the battery. Figure 41 

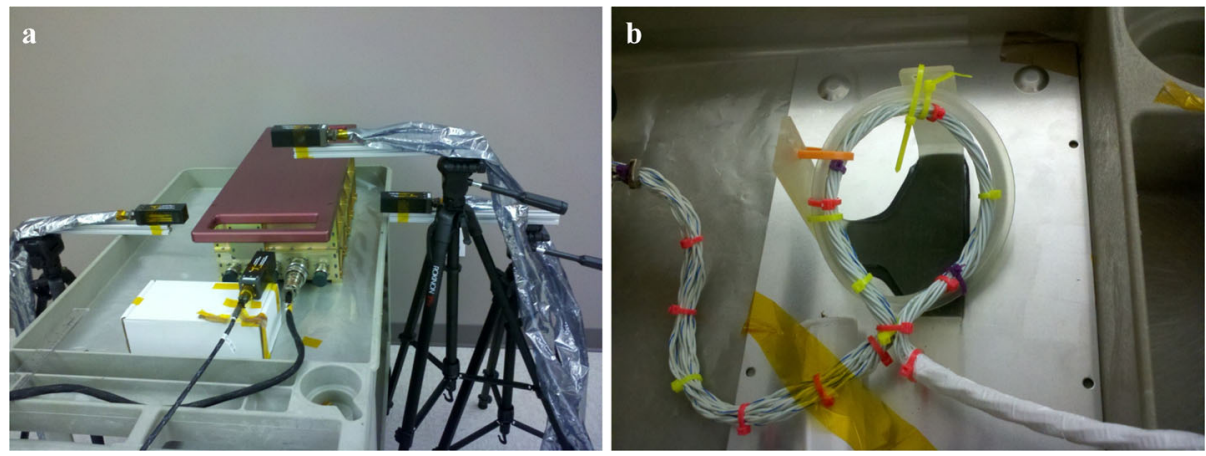

Fig. 40 Test sensor configuration for battery SN6 moment calibration (a), and customized harness current loop fabricated to cancel the battery current moment (b). Two distances were used to measure the battery moment and the closer distance configuration used is shown. The loop is 3.4 inches $(\mathrm{cm})$ in diameter and tilted so that the highest side is 1.4 inches $(\mathrm{cm})$ above the lowest. Return lines (white) and power lines (white with blue) are routed oppositely around the loop

Fig. 41 Battery loop polarity test results for SN6. Two sensors are used for this test, one above the battery loop (above) and the second above the harness loop (below). The vertical magnetic field ( $Z$-axis in blue) is opposite at the two sensors indicating the harness loop is properly oriented with polarity opposite the battery loop

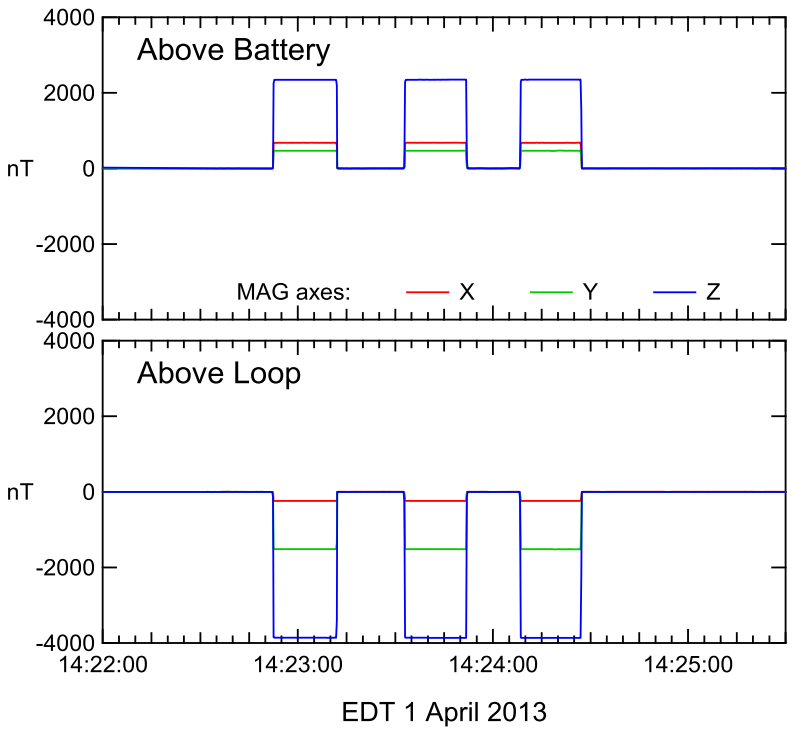

shows the results of this test for battery SN6, with a current of $11.6 \mathrm{~A}$, confirming that the right orientation was used. A second test to verify that the cancellation loop effectively cancels the battery moment was done using four magnetometers. The largest residual fields will be present along the line between the battery and the cancellation loop so the four magnetometers were positioned along this line in the direction away from the battery at distances ranging from $0.5 \mathrm{~m}$ to just over $1 \mathrm{~m}$ from the center point between the battery and cancellation loops. The results of this test for SN6, again using a current of $11.6 \mathrm{~A}$ are shown in Fig. 42. The data from the four magnetometers from closest (top) to furthest (bottom) are shown on the left and the log-log plot of the magnetic field versus distance from the midpoint between the battery and cancellation loops is shown on the right. The field decreases with distance faster than $1 / d^{3}$ and is consistent with a quadrupolar field, $1 / d^{4}$, indicating 

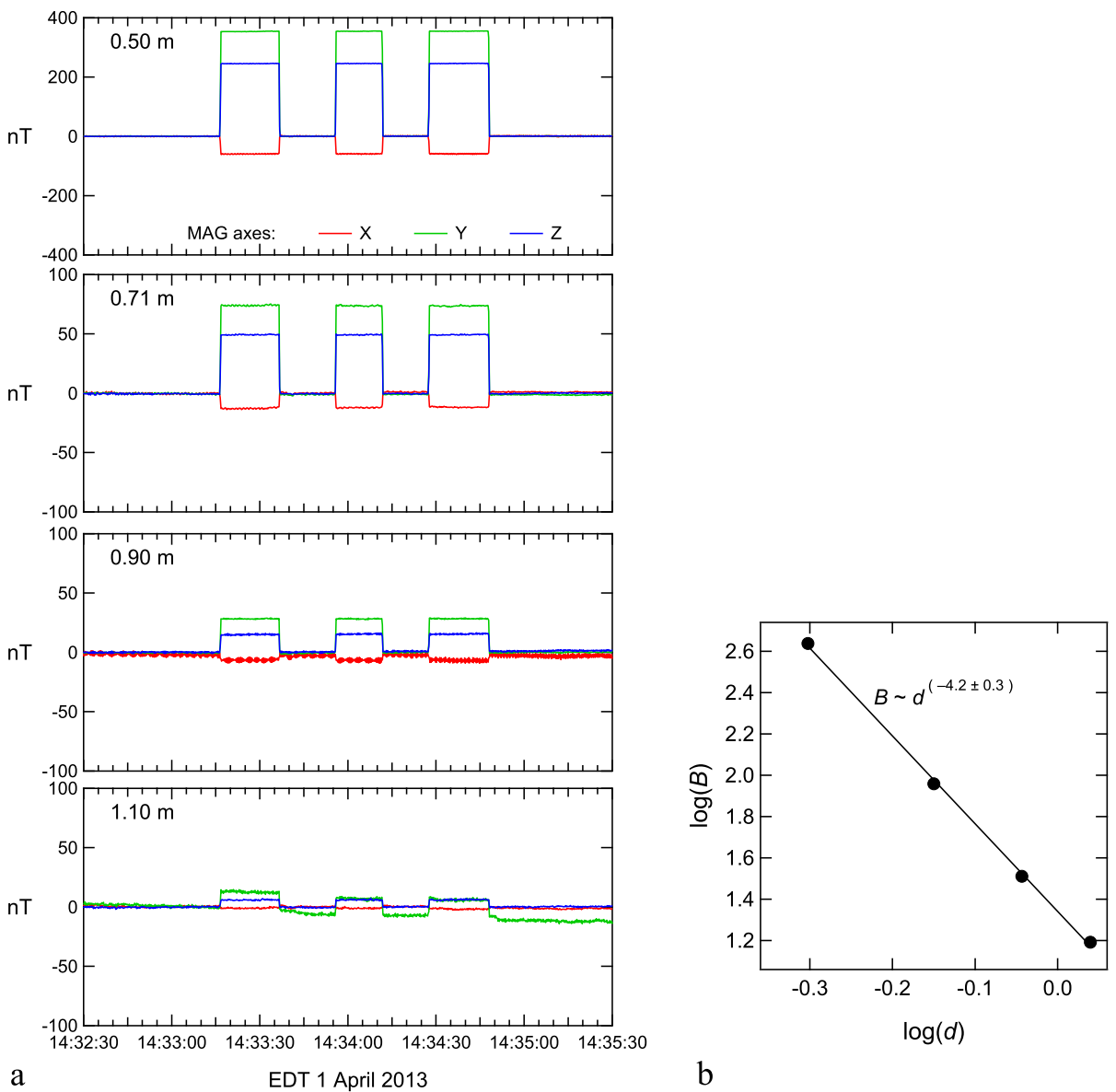

Fig. 42 Battery cancellation verification results for battery SN6. Data from four test magnetometers (a) ordered by distance from the mid-point between the battery and cancellation loops from closest (top) to furthest (bottom) showing the response for three intervals of $11.6 \mathrm{~A}$ current flow through the battery. The distance of each sensor is given in the corresponding panel. Magnetic field versus distance in a log-log plot with a power law fit is shown on the right (b). The power is consistent with a $1 / d^{4}$ scaling of the field expected for a quadrupole field

that the cancellation is correct. The residual dipole was estimated to be less than $5 \%$ of the original battery loop moment.

\subsubsection{Results: Margins and Outages}

All sub-systems, key components, and electronics units were tested and evaluated relative to their magnetics allocations. As noted, units exhibiting insignificant magnetic moments in tests of the engineering or first flight unit were not tested further and their allocations were released. For each unit, the upper limit moment estimate, $\mu_{i}$, after mitigation steps if necessary, was used to define the percentage margin as

$$
M_{i}=100\left(1-\mu_{i} / \mu_{\mathrm{Alloc}, i}\right)
$$


Table 6 Margins relative magnetics allocations for representative units on the MMS spacecraft

\begin{tabular}{lllll}
\hline Unit & DC-stability & & & \\
& & DC fixed & & Currents \\
\cline { 4 - 4 } Battery & Materials & Magnets & $99 \%$ \\
Solar array & $50 \%$ & $>90 \%$ & $(-15 \%)^{\dagger}$ & $>90 \%$ \\
Latch valves & n/a & $>90 \%$ & $\mathrm{n} / \mathrm{a}$ & $\mathrm{n} / \mathrm{a}$ \\
Transponder & $82 \%$ & $\mathrm{n} / \mathrm{a}$ & $29 \%$ & $82 \%$ \\
RF Switch & $\mathrm{n} / \mathrm{a}$ & $(-15 \%)$ & $\mathrm{n} / \mathrm{a}$ & $\mathrm{n} / \mathrm{a}$ \\
Power switching unit & $56 \%$ & $\mathrm{n} / \mathrm{a}$ & $(-450 \%)^{\ddagger}$ & $64 \%$ \\
CIDP & & $68 \%$ & $\mathrm{n} / \mathrm{a}$ & $73 \%$ \\
HPCA & $76 \%$ & $(-20 \%)$ & $\mathrm{n} / \mathrm{a}$ & $96 \%$ \\
EDI-GDU & $93 \%$ & $83 \%$ & $\mathrm{n} / \mathrm{a}$ & $(-210 \%)^{\ddagger}$ \\
SDP & $96 \%$ & $(-84 \%)^{\ddagger}$ & $\mathrm{n} / \mathrm{a}$ & $56 \%$ \\
DES & $43 \%$ & $(-155 \%)^{\ddagger}$ & $\mathrm{n} / \mathrm{a}$ & $62 \%$ \\
\hline
\end{tabular}

\footnotetext{
* Margins expressed as a percentage of allocation used. Values are the lowest margin of all units tested. All units with excess are listed here

${ }^{\dagger}$ Margins are negative if the allocation is exceeded and are enclosed in parentheses, ()

${ }^{\ddagger}$ Re-allocation of unused margins from other units was sufficient to cover excess
}

in each category, DC-stability, DC-materials/magnets, and DC-currents. For each of the 58 items on each observatory, 12 had margins over $80 \%$ in every category and 9 had margins under $20 \%$ in at least one category. Table 6 gives the margins for representative units, including those listed in Table 4 as well as the RF switch (mentioned above) and the inplane wire boom deployer unit (SDP). All cases in which the allocations were exceeded and not mitigated are given Table 6.

The RF switch moment exceeded the RF system DC allocation. The RF switch has two positions whose moments differ in direction such that the vector difference is more than half of the total moment. Thus, mitigation by cancellation was not an option. Because most units used a small fraction of their fixed field allocations, the RF switch outage was covered by reassigning some allocations from other units. This could not be done to meet the DC-stability requirement however since the change in RF switch moment violated its allocation by too large a factor. Thus, an operational constraint was adopted that the RF switch position not be changed during prime science observation intervals.

The more modest outages were dealt with by reassigning unused allocations of other units. The battery, CIDP, and transponder fixed field outages were particularly modest. The EDI outages for fixed fields due to materials and currents are due to the fact that the unit allocations are very small, owing to the fact that it is the closest unit to the electron beams it generates and receives. Thus, more extensive testing in the volume of the electron beam rather than near the electronics end (inboard) of the unit verified that the EDI-generated fields in the relevant volume are actually within its allocation even though the conservative moment determinations were not. Somewhat more problematic are the SDP overages in fixed moment which are substantial and result from magnets in the deployment mechanism motor. It turns out that these motors are mounted on the inbound end of the unit and accounting for the actual source locations reduces the outages enough to cover with unused allocations not already re-assigned to other units. 
There were no violations of the low frequency magnetics emissions. In the analysis for the amplitude spectra, there were two cases of violations in worst-case analysis, the HPCA noted already, and the combined electronics box (CEB) of the Fields investigation. More extensive testing using multiple test sensor positions to locate the emission source revealed acceptable levels demonstrating that the HPCA and CEB emissions were within their unit level allocations.

\subsection{Observatory Testing Program}

Three observatory-level magnetics tests were developed to ensure that the collective effects of all units remained under the observatory magnetics requirements. These tests were a validation of the electromagnetic interference test program and reduced risk to the project. This was conducted on the first observatory at a point in integration prior to installation of the solar arrays when the interior of the spacecraft was still accessible. The test was done as early as possible to reduce the impact of potential outages requiring mitigation steps. The second test was an EMI test of a fully integrated observatory which was designed to verify compliance with magnetic emissions due to currents and to assess the RF-switch transition fields. The third test was an unpowered DC magnetics test, conducted by displacing the spacecraft horizontally while it was suspended from an overhead crane. The unpowered test was designed to verify compliance of the fixed field due to materials and magnets with the observatory limits.

\subsubsection{Risk Reduction Test}

There were two objectives of the risk reduction test. First, to verify that the moments due to electric currents were as expected based on the unit-level testing and second to assess for the presence of chassis ground return currents. For the first portion of the test, magnetometers were positioned within and outside the spacecraft volume. The background noise was $\sim 2 \mathrm{nT}$ and only two locations exhibited signals above the $\sim 2$ nT background ambient variability, one within $30 \mathrm{~cm}$ of the battery (which was not configured with a cancellation loop for this test) and another within a few inches of the PSEES connector plane. No observatory outages were found in the EDI or HPCA bays, and no significant emissions were noted from the CIDP or CEB units, which service power to other payload sub-systems.

The second objective was to quantify any power return currents flowing through the chassis. A chassis ground return current corresponds to an equal deficit in the harness power return current. Hence, the chassis current measurement was done by measuring the magnetic field of any net current flowing in the power harness. Figure 43 shows the configuration of test magnetometers used for this test and illustrates the signature of a net harness current. Because this required access to the spacecraft interior, the measurement had to be done prior to installation of the solar arrays which form the side walls of the spacecraft.

Because the magnetometers positioned around the harness are also close to other spacecraft avionics, especially the navigator ultra-stable oscillators which are visible immediately behind the test magnetometers, the test analysis required discrimination between harness net currents and unrelated field gradients. To do this, we consider the change in magnetic field $\mathbf{d B}_{k}$ at the $k$ th magnetometer associated with power on or off of some unspecified unit. The net signal corresponding to a chassis current would be

$$
d B_{\text {Harness }}=\left(d B_{Z 1}-d B_{Y 2}-d B_{Z 3}\right) / 3
$$



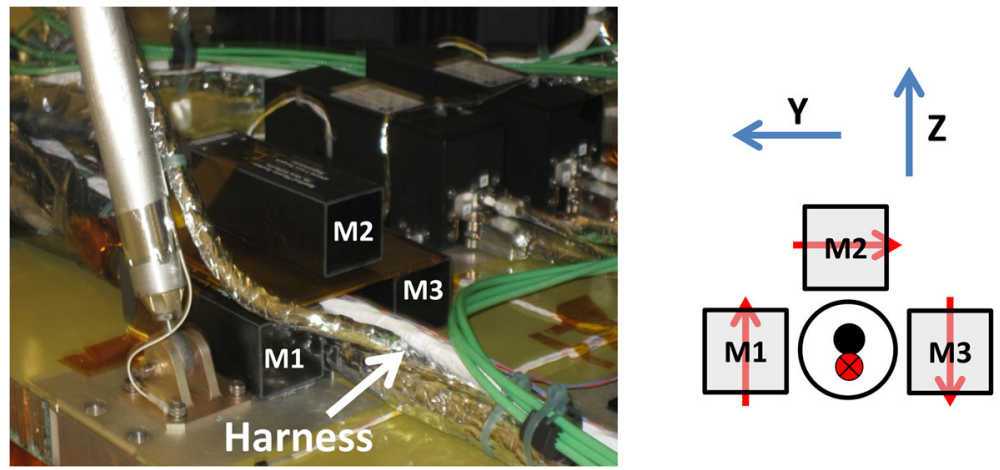

Fig. 43 Chassis current test configuration used during the risk reduction test. Arrangement of three test magnetometers on three sides of the harness (left) and the relationship of magnetometer $Y$ and $Z$ axes directions and magnetic field at the sensors due to a net current in the harness indicative of a ground return current (right). On the right, the red arrows indicate the magnetic field due to a net harness current flowing into the page, corresponding to a deficit of ground return current that necessarily occurs in the event of a chassis power return. Coordinate system is that of test setup

This is to be compared against the average gradients in directions unrelated to harness currents

$$
d B_{\mathrm{Grad}}=\operatorname{avg}\left\{d B_{X 1,2}, d B_{X 2,3}, d B_{X 1,3}, d B_{Y 1,3}, d B_{Z 13,2}\right\}
$$

Where $d B_{\mathrm{Cl}, m}$ denotes the absolute value of the difference in the $C$ component between magnetometers $l$ and $m$ and $d B_{Z 13,2}$ is the absolute value of the difference between $d B_{Z 2}$ and the average $d B_{Z}$ of magnetometers 1 and 3. The ratio $d B_{\text {Harness }} / d B_{\text {Grad }}$ was used to measure the degree of significance in the signal attributed to a harness current where values greater than 1 indicate clear detection of a harness current. The background noise level was $\sim 1 \mathrm{nT}$ and the corresponding minimum detectable chassis current for the geometry of the sensors was $\sim 0.07 \mathrm{~mA}$. Because the power harness from the PSEES splits into two branches close to the PSEES, the test was done on each branch of the harness separately powering the units in each branch. The total number of power on/off transitions was 41 for which only 20 yielded measureable steps in any of the three magnetometers and of these 20 only one gave $d B_{\text {Harness }} / d B_{\text {Grad }}>1$ while the average was 0.4 and this step corresponded to a chassis current no larger than $0.38 \mathrm{~mA}$. The sum of the absolute value of the estimated chassis current for all turn-on steps was $1.4 \mathrm{~mA}$ while the corresponding sum for all turn-off steps was $0.5 \mathrm{~mA}$. The total chassis current is therefore less than $1.4 \mathrm{~mA}$ and likely less than $0.5 \mathrm{~mA}$, well within the observatory limit.

\subsubsection{Electromagnetic Interference (EMI) Test}

The observatory EMI testing included extensive magnetic field testing to characterize the DC powered moments, DC stability, and AC low frequency by monitoring the magnetic fields near the observatory during subsystem power cycling and mode activation. The test configuration is illustrated in Fig. 44 and used two 4-magnetometer test systems. Specific targets of the testing were: battery charge and discharge, and the navigator (M-5, M-6, and M-7); the solar array connector, RF switch, transponder, and EDI-GDU (M-3 and M-4); and PSEES and EDI-GDU (M-8). Targets specifically for low frequency AC testing were HPCA and CEB (M-1 and M-2). 


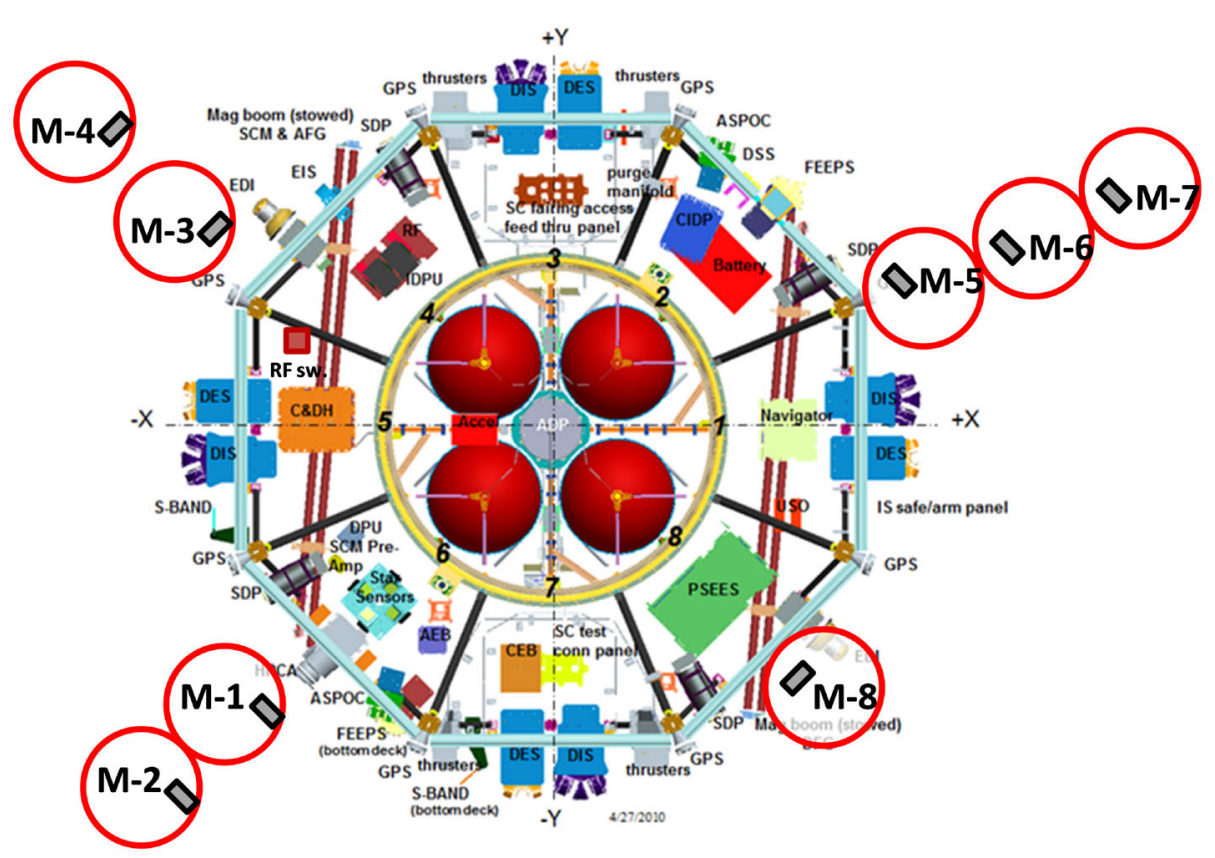

Fig. 44 MMS observatory schematic and positions of the eight test magnetometers, denoted 'M-n' and circled in red, used for the observatory EMI test. The observatory bays are numbered on the gold interior ring representing the cylinder housing the propulsion system fuel tanks (red). The EDI-GEU units are in bays 4 and 8 . The DES detectors are in the odd numbered bays. The digital fluxgate (DFG) boom root hinge is in bay 2 and the analog fluxgate (AFG) and search coil magnetometer boom root hinge is in bay 4

The EMI test consisted of background data acquisition followed by progressive power on of the observatory first through the avionics and then the payload. Data were then acquired for the low frequency magnetics test with operation in fast survey mode. The RF switch was actuated by switching from position A to B and then back to position A. The battery was then operated through charge and discharge modes. Finally, the payload and then the observatory were powered off. An overview of the magnetometer data from all eight test sensors is given in Fig. 45. For the first $\sim 70$ minutes of the test, the battery was charging at full charge current, until $\sim 00: 15$ GMT, when the charge current began to decrease as the battery reached a fully charged state. Only the RF switch operation and battery charge/discharge mode transitions gave detectable level shifts in any of the test sensors indicating compliance of the observatory powered moment requirements from all other systems. The battery relays will not be actuated during any science observations. Only the RF switch operation and battery charge/discharge mode transitions gave detectable level shifts in any of the test sensors indicating compliance of the observatory powered moment requirements from all other systems. Expanded views of the data for the RF switch operation and battery charge/discharge operations are shown in Fig. 46. The battery relays will not be actuated during any science observations.

All subsystem activations and mode changes were recorded and magnetic steps evaluated at each transition. Only battery charge/discharge state changes, RF switch operation, and navigator power on/off generated fields above the few nT standard deviation variability in the ambient field, but all of these were well within observatory limits. Transponder power on/off and transmission on/off were not detectable and no out-of-limit variations oc- 

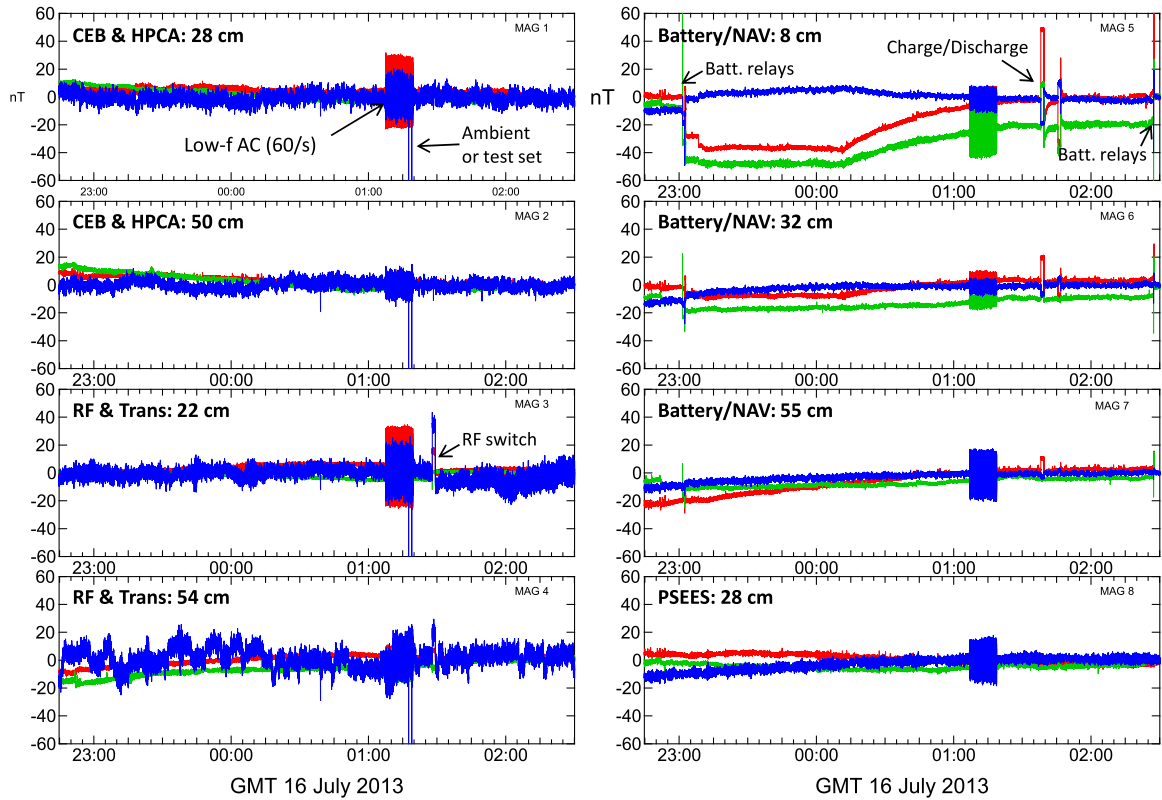

Fig. 45 MMS observatory \#1 EMI test overview results showing the entire time span of the test. Targets for each magnetometer are identified in each panel along with the distance outboard from the SC outer envelope. Key events during the test are also noted. The noise in the $Z$-component of magnetometers 1 through 4 was subsequently diagnosed as an improper ground connection affecting only the $Z$-axis channels of the test system for these magnetometers. The interval from 01:05 to 01:15 GMT was the 60/s sampling collection interval for the low frequency magnetics test

curred in association with EDI power on or operations or in either EDI-GDU bay due to any other event. The RF switch transitions correspond to changes well below the total field requirement for EDI but above the DC-stability requirement as expected. The battery transitions indicated a residual loop area of $0.0045 \mathrm{~m}^{2}$, within requirements, but larger than for a matched compensation loop, but as expected since the battery available for this test was not matched to the harness compensation loop. The test nonetheless confirmed the cancellation polarity of the flight harness.

During the low frequency emissions test from 01:05 to 01:15 GMT, no emissions above the observatory-level requirements were observed. The most likely sources were the HPCA and CEB units which were monitored by M-3 and M-4. Figure 47 shows the amplitude spectrum from M-3 for the test and for a background interval acquired after the observatory was powered down. The only emissions above the observatory requirement are discrete peaks attributed to ambient noise. No emissions are evident other than a broad peak near $13 \mathrm{~Hz}$ not attributed to either HPCA or CEB and which is consistent with varying background contamination due to the fan systems in the EMI clean room facility. Neither EDI-GDU bay exhibited emissions above limits.

\subsubsection{Unpowered Fixed Field Test}

The unpowered spacecraft magnetic field test was conducted by suspending the spacecraft from a high overhead crane in the tallest clean room integration facility at GSFC. The lifting assembly used an Aluminum load distribution fixture and Nylon straps to ensure that 

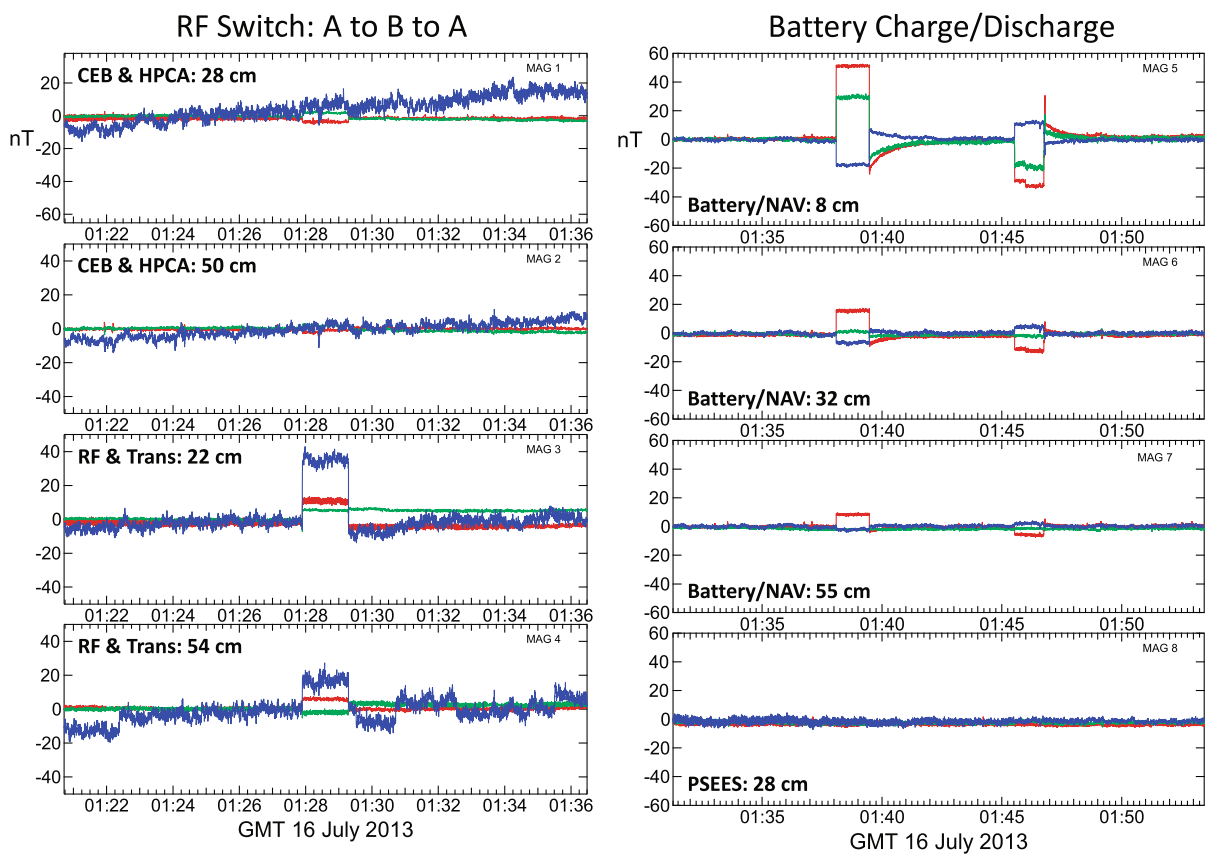

Fig. 46 MMS observatory \#1 EMI test zoom-in views for the RF switch actuation (left) and the battery charge/discharge sequence (right)

Fig. 47 MMS observatory \#1 EMI test low frequency AC results for the test magnetometer closest to HPCA and CEB. Red trace shows data with both units on and in fast survey mode while gray shows data with the observatory powered off. The observatory requirement is shown in the heavy gray line

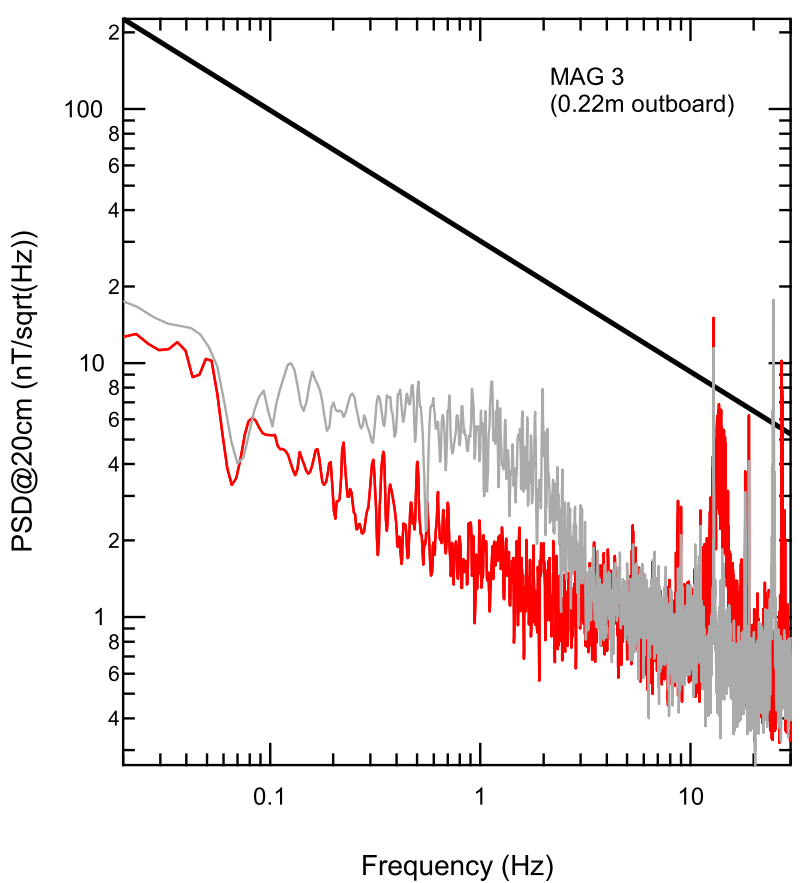

Springer 

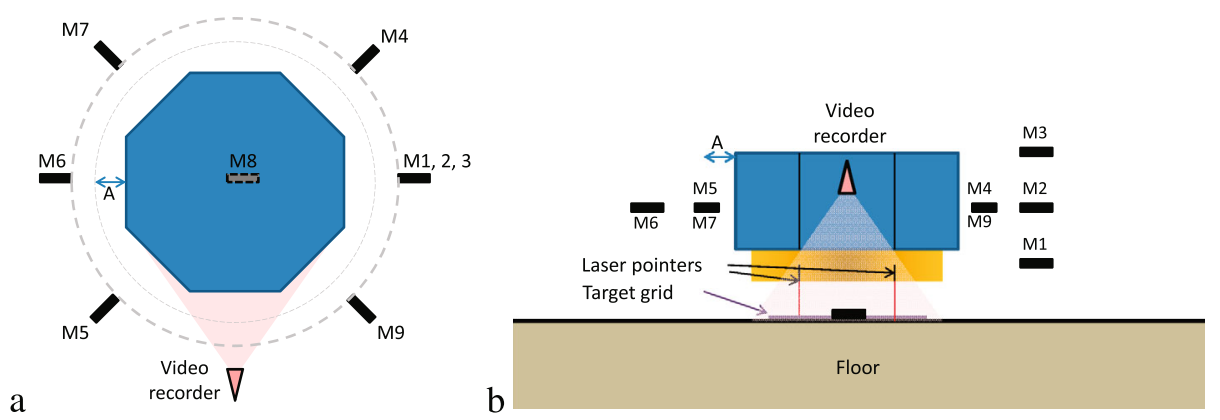

Fig. 48 Test configuration for MMS observatory unpowered magnetics test showing the placements of 9 test magnetometers, laser pointers, and video recorders used view from above (a) and from the side (b). A target grid was placed on the floor to track spacecraft displacement from equilibrium during the test

the crane hook was as high as possible to minimize magnetic interference. Non-magnetic manacles and lift fixtures were acquired or manufactured, respectively, to transfer the lifting load from the straps to the MMS separation ring. Both the fixtures and the manacles were verified to be free of detectable magnetization within inches from a test magnetometer at a level of $\sim 5 \mathrm{nT}$. The spacecraft was lowered into the testing area configured as shown in Fig. 48 using 9 test magnetometers, a video recorder, and laser pointers. Data were recorded as the spacecraft was lowered into position and the test consisted of controlled swings of the spacecraft in the direction indicated by 'A' in Fig. 48. Displacements of 20 to 30 inches were used and three to four successive quasi-free swings were performed while monitoring spacecraft motion using the video recorder tracking the laser pointer projections to a grid resting on the floor. Between lateral displacement series, the spacecraft was rotated $45^{\circ}$ about the vertical direction so that at least one set of lateral displacements were performed with every face of the spacecraft toward M2.

Data from M2 are shown in Fig. 49 when the spacecraft was lowered into position (a) and for a representative set of swings, corresponding to the EDI-GDU bay that also hosts the PSEES (b). The radial field component is a factor of 3 to 4 larger than the vertical or tangential field components indicating that the moment is predominately in the horizontal plane and radial. The equilibrium distance of the spacecraft center from the test magnetometers was $2.8 \mathrm{~m}$ on average. The data from the swings were analyzed by recording the displacements at swing extrema and examining the variation versus $1 / d^{3}$ where $d$ is the distance to the center of the spacecraft. If $B_{1}$ is the magnetic field in the displaced position, $d_{1}$, and $B_{0}$ is the magnetic field at the equilibrium position, $d_{0}$, then one can derive $B_{0}$ from a set of $B_{1}$ and $d_{1}$ values using

$$
B_{1}-B_{0}=B_{0}\left(d_{0} / d_{1}\right)^{3}-B_{0}
$$

where $d_{0}, d_{1}$, and $B_{1}-B_{0}$ are the measured quantities so $B_{0}$ is determined from a simple fit to. The results of this for the radial component from the complete test are shown in Fig. 50 which also shows the multipole fit and the dipole portion of the fit to the data. The polar angle corresponds to spacecraft bays 1 through 8 from left to right, where $\theta=0$ is bay 1 and $\theta=\pi$ is bay 4 . These values correspond to distance from the spacecraft outer edge of $1.1 \mathrm{~m}$. A dipole field would give a single cosine wave and it is evident that the field is predominantly higher order, presumably because the sources are located in each bay.

The results demonstrate compliance with the requirements (cf. Table 3). Note that the distances in Table 3 are measured relative to the corresponding sensor and not from the center 
Fig. 49 Data recorded by M2 before the test as the spacecraft was lowered into position (a) and during one of the displacement series (b). The $X$-axis is positive horizontal and radially away from the spacecraft, the $Y$-axis is positive to the right looking from spacecraft, and the $Z$-axis is upward the magnetometer toward the
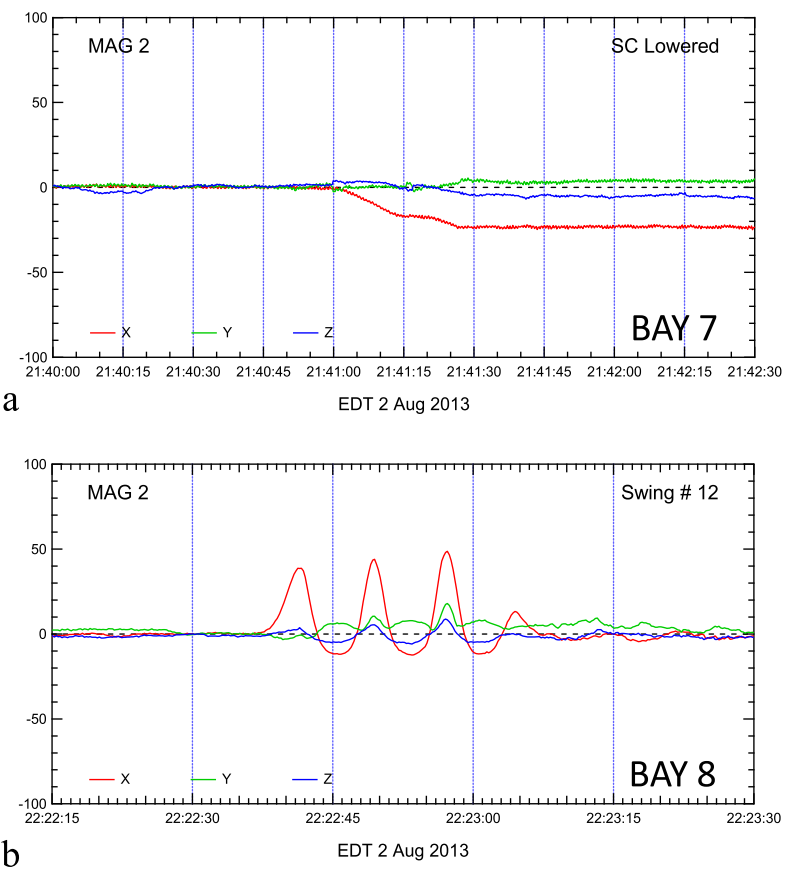

Fig. 50 Radial magnetic field at 2.81 meter distance from spacecraft center as a function of angle around the perimeter of the spacecraft where $\theta=0$ and $\theta=\pi$ correspond to bays 1 and 5, respectively. Solid gray line shows a multipole fit (through octupole) to the data and the dashed black line shows the dipole component of the fit

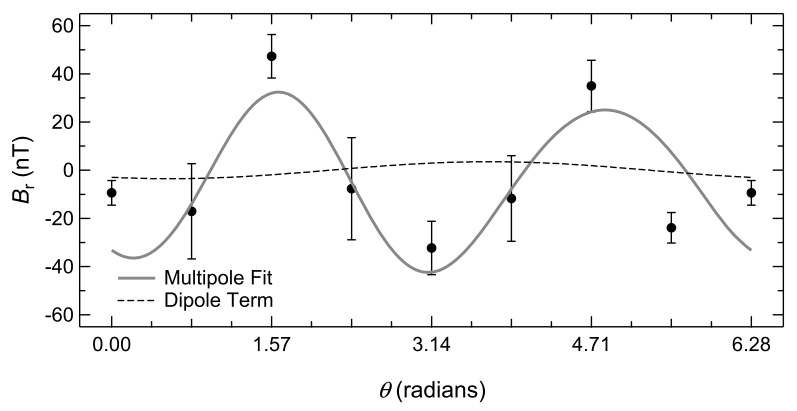

of the spacecraft. The dipole moment from the fit is $0.40 \mathrm{~A}-\mathrm{m}^{2}$ which is measured from the center of the spacecraft. In the EDI bays, 4 and 8 , scaling the observed field to the $1.5 \mathrm{~m}$ distance using $1 / d^{3}$ scaling (an upper limit) gives an estimated radial field of $15 \mathrm{nT}$, within the 24 nT requirement. To judge compliance with the requirements of Table 3 more generally, the multipole fit was used to estimate the field at the distances from the spacecraft center as shown in Fig. 51 together with the appropriate distances for DES, EDI and AFG/DFG. This curve simply adds the magnitudes of the dipole, quadrupole, and octupole terms with $1 / d^{3}$, $1 / d^{4}$, and $1 / d^{5}$ scaling without regard for the angular structure in the field and is therefore a conservative upper limit. All requirements are met even with this conservative estimate.

\subsection{Assessment}

The magnetic cleanliness program relied on quantitative flow-down of observatory level requirements to units and subsystems. By adopting a realistic and not overly conservative 
Fig. 51 Multipole fit result for the upper limit radial magnetic field due to permanent magnetization of MMS observatory \#1 versus distance from spacecraft center. The relevant SC center distances for the DES, EDI, and AFG/DFG requirements are indicated by vertical gray lines. The EDI and AFG/DFG requirements are indicated by arrows. The DES requirement is $1200 \mathrm{nT}$ and is off scale above the graph

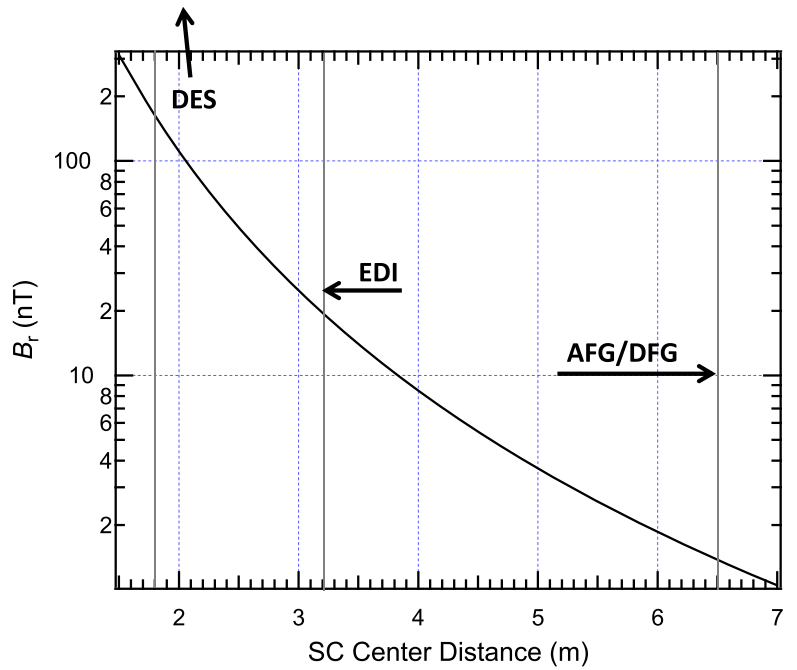

allocation approach, the designs were informed in a way that allowed accommodation using effort focused on the critical subsystems while ensuring that all potential magnetic field sources were identified and scrutinized. The success of the magnetic cleanliness program depended on a comprehensive review of electrical systems designs and wiring layouts, and institution of a consistent, comprehensive, and feasible program of testing. A number of items were identified prior to fabrication that required particular attention including the solar arrays, battery, propulsion latch valves, and power switching electronics. The mitigation steps of these units were largely successful and outages were identified in testing allowing minor adjustment to solar array wiring and mitigation of a significant battery current loop using an additional harness loop. The wire boom deployers (SDP units) and RF switch exhibited the greatest outages relative to their allocations. The success in the magnetics design of the remainder of units allowed for the accommodation of these excesses by reassigning unused allocations of other units to inform the decision not to pursue mitigation in these cases. Observatory testing of magnetic emissions during powered operations and due to the spacecraft permanent moments confirms that the magnetics requirements were met and ensured that the contamination fields of the MMS spacecraft will not present constraints to the science magnetic field measurement objectives.

\section{Magnetic Field Measurements Data Flow}

\subsection{Overview}

The AFG/DFG data must be processed in a timely and accurate manner to meet the varied needs of the MMS mission. The MMS project requires that all instruments release Quicklook (QL) data products to the science community within 24 hours of receipt of the raw data, and Level 2 science-grade data sets within a month (although Level 2 data products may be re-processed if calibrations can be significantly improved after observing long-term trends in the data). In addition, however, AFG/DFG data products are used for operations of the burst data management system, and are critical inputs to data processing and operations of other instruments. These requirements drive the design of a system of data levels, 
Table 7 AFG/DFG Data levels and delivery schedule, in terms of Time after Receipt (TAR) of data by the Science Data Center. Note that receipt of burst data is delayed by a few days relative to survey data; hence delivery of burst data will be correspondingly delayed

\begin{tabular}{lllll}
\hline $\begin{array}{l}\text { Data } \\
\text { Level }\end{array}$ & $\begin{array}{l}\text { Time of } \\
\text { Delivery } \\
\text { (TAR) }\end{array}$ & $\begin{array}{l}\text { Coordinate } \\
\text { System }\end{array}$ & Description & Intended User \\
& & & \\
\hline
\end{tabular}

\begin{tabular}{|c|c|c|c|c|}
\hline $\begin{array}{l}\text { Level 1A } \\
\text { (L1A) }\end{array}$ & 5 minutes & AFG123/DFG123 & $\begin{array}{l}\text { Time-taged, uncalibrated } \\
\text { pseudo-nT }\end{array}$ & Cal., Higher level processing \\
\hline $\begin{array}{l}\text { Level 1B } \\
\text { (L1B) }\end{array}$ & 10 minutes & BCS, OMB & $\begin{array}{l}\text { Last Available Cal., spinning } \\
\text { SC Coords }\end{array}$ & $\begin{array}{l}\text { EDI, E-Fields, AFG/DFG } \\
\text { Quicklook processing }\end{array}$ \\
\hline $\begin{array}{l}\text { Quicklook } \\
\text { (QL) }\end{array}$ & 20 minutes & $\begin{array}{l}\text { DMPA, } \\
\text { GSM-DMPA }\end{array}$ & $\begin{array}{l}\text { Last Available Cal., desupn } \\
\text { SC Coords., near geophysical }\end{array}$ & $\begin{array}{l}\text { Scientist in the Loop, Science } \\
\text { Community }\end{array}$ \\
\hline $\begin{array}{l}\text { Prelim } \\
\text { Level } 2 \\
\text { (L2pre) }\end{array}$ & 14 days & $\begin{array}{l}\text { BCS, DMPA, } \\
\text { GSE }\end{array}$ & $\begin{array}{l}\text { Preliminary Cal., SC \& } \\
\text { Geophysical Coords }\end{array}$ & $\begin{array}{l}\text { Particles, EDI and E-Fields } \\
\text { Level } 2 \text { processing }\end{array}$ \\
\hline $\begin{array}{l}\text { Level } 2 \\
\text { (L2) }\end{array}$ & 26 days & $\begin{array}{l}\text { BCS, DMPA, } \\
\text { GSE }\end{array}$ & $\begin{array}{l}\text { 'Best' cal., SC \& } \\
\text { Geophysical Coords }\end{array}$ & $\begin{array}{l}\text { Science Community; EDI, } \\
\text { E-Fields, Level } 2+\text { and Level } \\
3 \text { processing }\end{array}$ \\
\hline
\end{tabular}

whose need-by 'dates' range from minutes to weeks after receipt of raw data. Data levels and their delivery schedules are summarized in Table 7 . This section will discuss the flow of data and the best available calibrations to create the data products at each of these levels. Table 8 defines the principal coordinate systems of interest for AFG/DFG data processing and analysis, including some specialized systems, which will be introduced in this section.

The need for preliminary AFG/DFG products on short time scales is driven by the requirements of the MMS Burst Management system and the requirements of other instruments that are dependent on magnetic field data.

The MMS Burst Management system makes provision for a Scientist in the Loop (SITL), who has a short window of time between downlink of survey data and the next contact in which to validate and possibly override burst management decisions, based on preliminary science data products. Not only is AFG/DFG data required by the SITL, but also AFG/DFG data is required as an input for other FIELDS instrument SITL data processing, particularly for the spin-axis component of the DC E-field (DCE). The former requirement is met by processing AFG/DFG Quicklook data products within 20 minutes after the raw data is received (leaving plenty of time to make the same products available to the science community within 24 hours). The latter requirement is met by producing an intermediate, Level 1B product, within 10 minutes. This delivery schedule does not allow time for re-calibrating the magnetometers. Because time is short and the applications do not demand high accuracy, there are no specific requirements for accuracy on the Level 1B (L1B) and Quicklook data products.

Preliminary Level 2 data products must be made available within 2 weeks in order to be available for the production of particles, EDI and E-field Level 2 data products. The goal of preliminary Level 2 data product is to provide the magnetic field vector with an accuracy of 1 degree and field magnitude with $1 \%$ accuracy. In small fields where the magnetic field is less than $50 \mathrm{nT}$, the goal is to achieve $0.5 \mathrm{nT}$ accuracy.

Finally, best-effort AFG/DFG Level 2 data products are due by 26 days, making it possible for the FIELDS suite to combine data from multiple instruments on a single observatory to produce Level $2+$ data products within 30 days. Examples of Level $2+$ data products include a combined AFG/DFG and SCM data product, and an E-fields data product that re- 
Table 8 Coordinate systems, as used to describe AFG/DFG instrument data

\begin{tabular}{|c|c|c|}
\hline Coordinate System & Abbreviation & Definition \\
\hline Sensor Coordinates & $\begin{array}{l}\text { AFG/DFG } \\
\text { XYZ }\end{array}$ & $\begin{array}{l}\text { Non-orthogonal sensor coordinates, named according to } \\
\text { instrument chassis axes }\end{array}$ \\
\hline $\begin{array}{l}\text { Standardized Sensor } \\
\text { Coordinates }\end{array}$ & $\begin{array}{l}\text { AFG123 } \\
\text { DFG123 }\end{array}$ & $\begin{array}{l}\text { Non-orthogonal sensor coordinates, re-named according to the } \\
\text { following standard directions relative to the observatory: } \\
\text { Axis } 1 \text { points radially outward along the AFG boom } \\
\text { Axis } 3 \text { points along the positive spin axis }\end{array}$ \\
\hline $\begin{array}{l}\text { Orthogonalized } \\
\text { Magnetometer Boom }\end{array}$ & OMB & $\begin{array}{l}\text { Orthogonal coordinates } \\
X \text {-axis in plane defined by nominal AFG boom orientation and the } \\
\text { MPA, positive radially outward along the AFG boom } \\
Z \text {-axis aligned with MPA }\end{array}$ \\
\hline $\begin{array}{l}\text { Body Coordinate } \\
\text { System }\end{array}$ & BCS & $\begin{array}{l}\text { Spacecraft-fixed coordinates } \\
X-Z \text { plane crossing defines the sun pulse (positive } X \text { ) } \\
Z \text {-axis is nominal spin axis }\end{array}$ \\
\hline $\begin{array}{l}\text { Spinning, Major } \\
\text { Principal Axis } \\
\text { (MPA) }\end{array}$ & SMPA & $\begin{array}{l}\text { Spacecraft-fixed coordinates } \\
X \text {-axis is in plane defined by BCS } X \text {-axis and the MPA, } \\
\text { perpendicular to the MPA } \\
Z \text {-axis is the MPA }\end{array}$ \\
\hline $\begin{array}{l}\text { Despun, Major } \\
\text { Principal Axis }\end{array}$ & DMPA & $\begin{array}{l}\text { Near GSE } \\
X \text {-axis in plane defined by Earth-Sun line and MPA, perpendicular } \\
\text { to MPA, positive towards the Sun } \\
Z \text {-axis aligned with MPA, positive towards Ecliptic north }\end{array}$ \\
\hline $\begin{array}{l}\text { Near GSM, rotated } \\
\text { from DMPA }\end{array}$ & $\begin{array}{l}\text { GSM- } \\
\text { DMPA }\end{array}$ & $\begin{array}{l}\text { GSE to GSM transformation applied to DMPA coordinates } \\
X \text {-axis is same as DMPA } X \text {-axis } \\
Z \text {-axis is MPA rotated by GSE-to-GSM angle about } X \text {-axis }\end{array}$ \\
\hline $\begin{array}{l}\text { Geocentric Solar } \\
\text { Ecliptic }\end{array}$ & GSE & $\begin{array}{l}X \text {-axis is Earth-Sun vector } \\
Z \text {-axis is Ecliptic North Pole }\end{array}$ \\
\hline $\begin{array}{l}\text { Geocentric Solar } \\
\text { Magnetospheric }\end{array}$ & GSM & $\begin{array}{l}X \text {-axis is Earth-Sun vector } \\
Z \text {-axis is projection of Earth's magnetic dipole on the GSE } Y-Z \\
\text { plane }\end{array}$ \\
\hline
\end{tabular}

quires input from the entire FIELDS suite of instruments. In addition, Level 2 products will be used to create Level 3 data products, defined to be data products that contain parameters that can only be calculated by combining data from multiple observatories.

A top-level diagram showing the interaction of AFG/DFG data processing and calibration data flow is shown in Fig. 52. The first step in data processing is Level 1A processing, which is performed as part of the near real-time processing data flow that culminates in Level 1B and Quicklook data. Level 1A data is the basis for all calibration and down-stream data processing. Level 1A and all higher-level data products are in Space Physics Data Facility Common Data Format (CDF) files. Near real-time processing is performed automatically at the MMS Science Data Center (SDC), located at the Laboratory for Atmospheric and Space Physics (LASP) in Boulder, Colorado. The SDC is responsible for distributing data products to the SITL, to other instrument teams, and to the science community. Preliminary calibration data products, produced by GSFC, are uploaded to the SDC on a weekly schedule, providing the latest available calibrations to be applied to new data. These calibrations available at the SDC have epochs 5 to 14 days before the current data. Each day, the SDC 


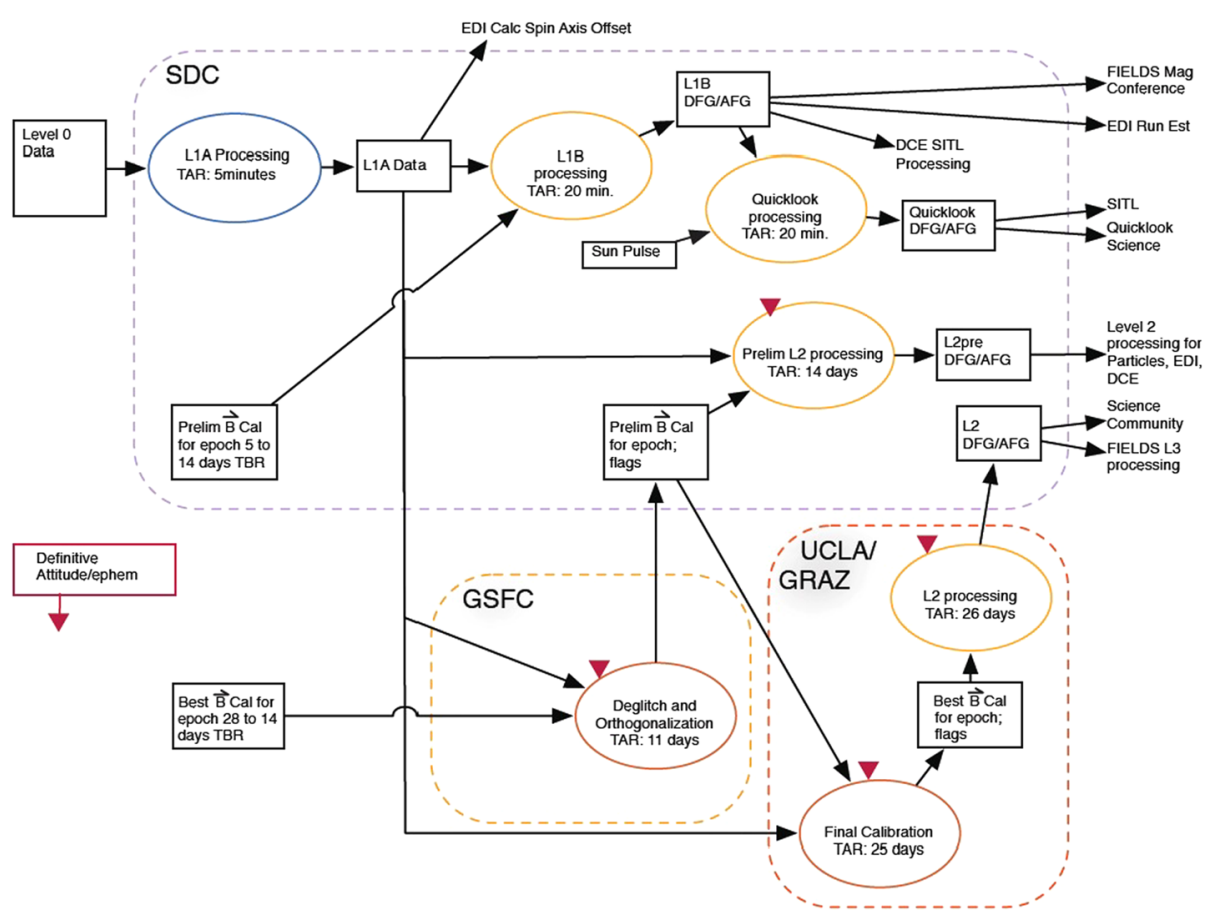

Fig. 52 Top-level diagram of AFG/DFG data processing and calibration data flow. The TAR designation in each process indicates the time by which the process must be complete, after new data is received. The epochs of the best available calibrations, relative to time the data being processed was received, are labeled as Time Before Receipt (TBR)

performs preliminary Level 2 processing, automatically applying the corresponding preliminary calibrations to data received 14 days in the past. If, due to unforeseen circumstances, corresponding preliminary calibrations are not available, preliminary Level 2 processing proceeds with the latest available calibrations. This arrangement allows for other instruments' Level 2 processing to commence on schedule, using reasonably good magnetic field data. GSFC, IWF-Graz, and UCLA perform the calibration steps described in Sect. 4.2 using semi-automated processes, but always with human interaction to verify the calibrations. Each institution uses Level 1A data as a starting point for their process, passing along an improved version of the calibration as an input to the next step. The resulting 'best' calibration is used for Level 2 processing at UCLA, and then becomes the starting point for the preliminary calibration of new data back at GSFC.

\subsection{Near Real-Time Data Processing}

The chain of L1A, L1B and Quicklook processing, shown in Fig. 53, meets the data processing requirements for the SITL as well as Quicklook. A single script controls the data flow, ensuring that necessary ancillary data is processed before the instrument data and that the correct data is routed into each of the instrument data processes. For the SITL, the SDC automatically triggers this script for each observatory, instrument, and mode (burst or survey), as soon as all data from a contact is available. Because the SITL product is simply an initial version of the Quicklook product, the Quicklook processing is the same, except that the SDC 


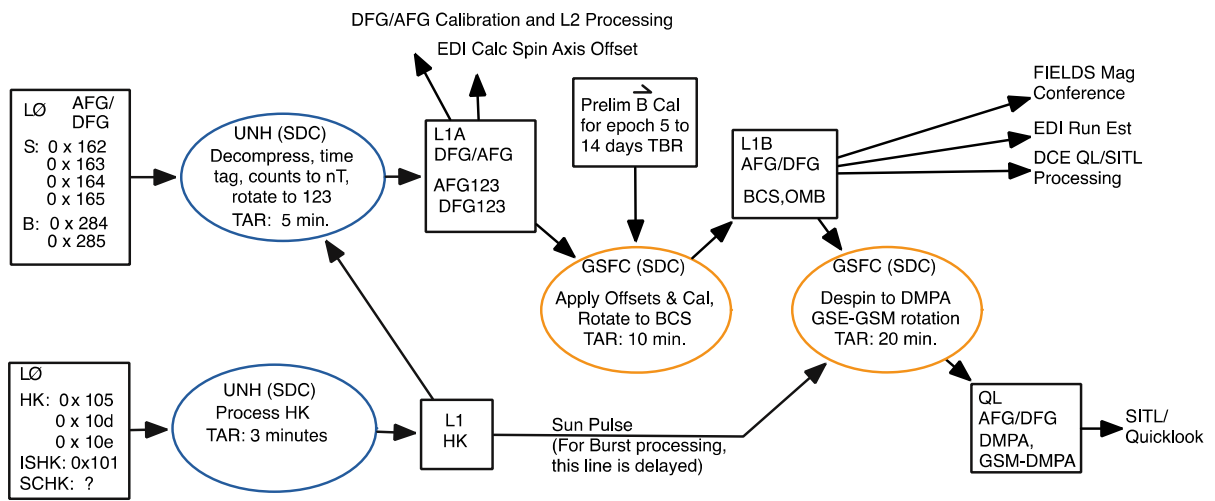

Fig. 53 L1A/L1B/Quicklook near-real-time processing data flow. In this and subsequent figures, the heading for each process indicates the institution responsible for creating the software, and in parenthesis, the institution where the process is run

first ensures that all data for a given day or burst segment has been downlinked, and then calls the same script, thus producing a 24-hour Quicklook survey file, or a single Quicklook burst file for each burst segment. UNH provides the Level 1A software and the script, while GSFC provides the L1B and Quicklook processing software. Level 1A software extracts data from the raw telemetry, calculates accurate time tags and converts from raw counts into pseudo-nT or appropriate engineering units. In addition, the L1A software transforms from AFG/DFG sensor coordinates, which point in different directions relative to the spacecraft, into standardized (but still non-orthogonal) sensor coordinates, which are co-aligned, to the first order. See Fig. 12 of the FIELDS instrument paper for an illustration of these systems. Definitive attitude/ephemeris is not available at the time of processing; however, sun pulse times are extracted from Instrument Suite housekeeping data that is downlinked at the same time as the instrument data. For newly received burst data, the associated ancillary data will have been downlinked previously, and the UNH script takes care of routing it to the Quicklook process. Level 1B processing uses calibration parameters which have been determined from previous data. The latest values are held constant, without trending or extrapolation. The process for updating the calibration files will be discussed in Sect. 6.3. Although housekeeping data with AFG/DFG sensor temperatures is available at the time of L1B processing, the temperature corrections described in Sect. 4.1.4 will not be applied at this level.

The L1B data files provide calibrated, orthogonalized data in the Orthogonalized Boom System (OMB), which is closely aligned to the actual sensor orientations and oriented in a precisely-defined manner relative to the spacecraft. L1B data files also include data in the Body Coordinate System (BCS), which provides a common reference frame for other instruments mounted to the spacecraft, such as EDI. Coordinate system definitions are summarized in Table 8. Quicklook data files include data in Despun, Major Principal Axis (DMPA) aligned coordinates and GSM-DMPA coordinates, which can generally be interpreted to be near the GSE and GSM geophysical coordinate systems, respectively. The data have been transformed with simple rotations, to avoid mixing of the spin-plane components with the spin-axis component. Thus, artifacts of the preliminary calibration can readily be distinguished from scientifically meaningful results. The DMPA system may be considered to be nearly GSE coordinates due to the fact that the spin axis is maintained by the mission to be between 2.5 to 5 degrees sunward of ecliptic normal while the observatories are in the 


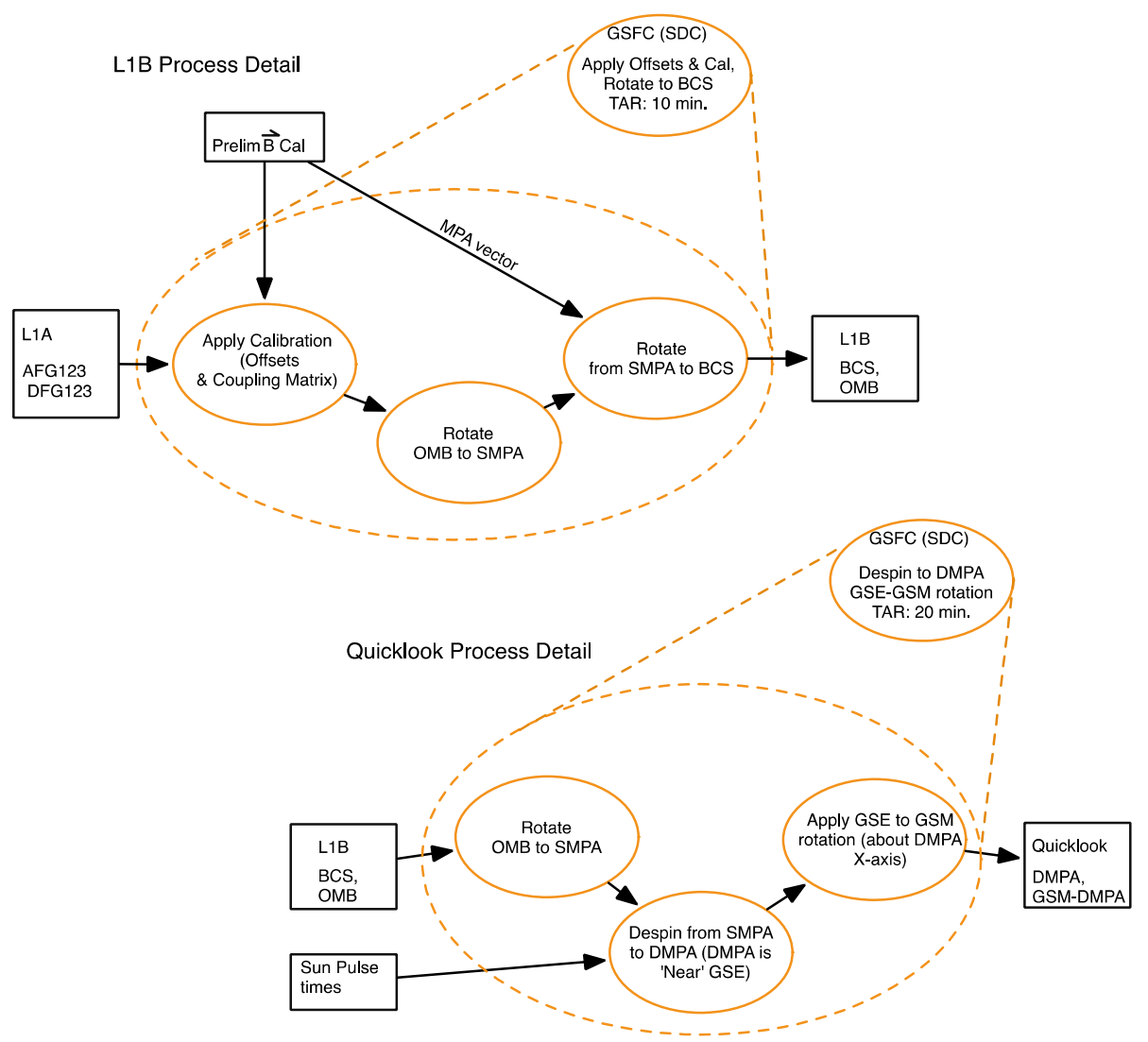

Fig. 54 Detail showing L1B and Quicklook calibration and coordinate transformation sub-processes

scientific region of interest. To provide a near GSM data product, the Quicklook process applies a GSE to GSM transformation to the DMPA data. Note that this is simply a rotation about the DMPA $X$-axis, so that in the resulting system, dubbed GSM-DMPA, the nearly sunward-pointing $X$-axis only includes data from the spin plane of the DMPA system.

The full sequence of transformations in L1B and Quicklook processing is illustrated in Fig. 54. The initial calibration step yields results data in the Orthogonalized Boom Coordinates (OMB). The OMB $Z$-axis is aligned with the MPA, while the $X$-axis is in the plane of the MPA and the nominal location of the radially-outward AFG boom vector. The rotation between OMB and SMPA is the same as the rotation between the nominal AFG boom position and the BCS $X$-axis (225 , as shown in Fig. 12 of the FIELDS instrument paper). Finally, the transformation from SMPA to BCS rotates by the angle between the MPA and the BCS $Z$-axis, in the plane containing the MPA and the BCS $Z$-axis. Note that the MPA vector, which is actually a product of the definitive attitude, is taken from the magnetometer calibration file, so that the L1B process does not require the definitive attitude file as an input. The Quicklook process begins with the OMB data from the L1B file, makes the OMB to SMPA transformation, and then uses the sun pulse times, which are essentially the times when the sun is in the SMPA $X-Z$ plane, to despin the data into DMPA coordinates. 


\subsection{Calibration}

The responsibility for carrying out the in-flight calibration activities described in Sect. 4.2 is divided between GSFC, UCLA and IWF-Graz. GSFC's primary responsibility will be to orthogonalize the sensor triads, i.e., to minimize the spin tone (Sect. 4.2.1). As a necessary pre-processing step, GSFC will vet the data for glitches and create a 'B Flag' file, which can be used to produce a clean data set. IWF-Graz will lead the effort to determine the magnetometer spin axis offset using data from EDI (Sect. 4.2.3). UCLA will be responsible for routine removal of jumps at range changes (Sect. 4.2.2), Earth field comparison (Sect. 4.2.5), and inter-spacecraft calibration (Sect. 4.2.6). In addition to these routine activities, UCLA will also be responsible for calibration in the solar wind (Sect. 4.2.4) using Alfvenic fluctuations. Calibration algorithms are provided by UCLA and Graz, while the software design and implementation is a joint effort.

The DFG/AFG team is responsible for producing science-grade Level 2 fluxgate data within 26 days of Time After Receipt (TAR) of the Level 0 data. Each calibration technique must be applied in sequence. A possible timeline that allocates time for each of the routine calibration processes is shown at the bottom of Fig. 55. The timeline takes into consideration the following questions:

1. How much processing time (human and automated) is needed after the last data to be used in processing has been received?

2. How much data are needed beyond the epoch of the data to be delivered, in order to perform the necessary processing/trending/analysis?

3. What delivery schedule can be reasonably maintained, given weekends and other responsibilities?

Calibration activities generally proceed at a weekly cadence, where a single calibration technique is applied on one week's data, before the results are stored in an intermediate calibration file that is passed on to the next step. Note that in this particular scheme, the Earth field and inter-spacecraft calibration processes are only able to look ahead one week with preliminary calibrations, although further look-ahead would be possible with L1B-quality data. At the time of writing, it is clear that the data flow and timeline may evolve significantly during the actual mission. The 26-day deadline only becomes effective 6 months after the end of commissioning. The process will be adapted, practiced and improved during the intervening months.

The activities of the various institutions are coordinated at weekly 'Mag Conferences'. Data for a given week will be discussed at least three separate Mag Conferences, labeled 'A', 'B' and 'C' in Fig. 55, before it is ready for delivery as Level 2 data. Looked at another way, the weekly Mag Conference would have three sections, with each section discussing data from successively earlier epochs.

The objectives of Mag Conference 'A', held within 12 days after receipt of the data, are:

1. Review the detailed calibration parameters from GSFC for the week of data which is 12 to 6 days in the past.

2. Review the data flags, ensuring that bad data have been flagged, and that good data are not flagged. GSFC will be responsible for taking corrective action based on the findings of the conference.

3. Discuss whether it is appropriate to apply temperature correction in Preliminary Level 2 processing, using filtered sensor temperatures from the instrument housekeeping data.

4. Determine the set of calibrations that should be released in a new version of the 'Prelim B Cal' file. This file will be delivered to the SDC, where the calibrations will be applied 


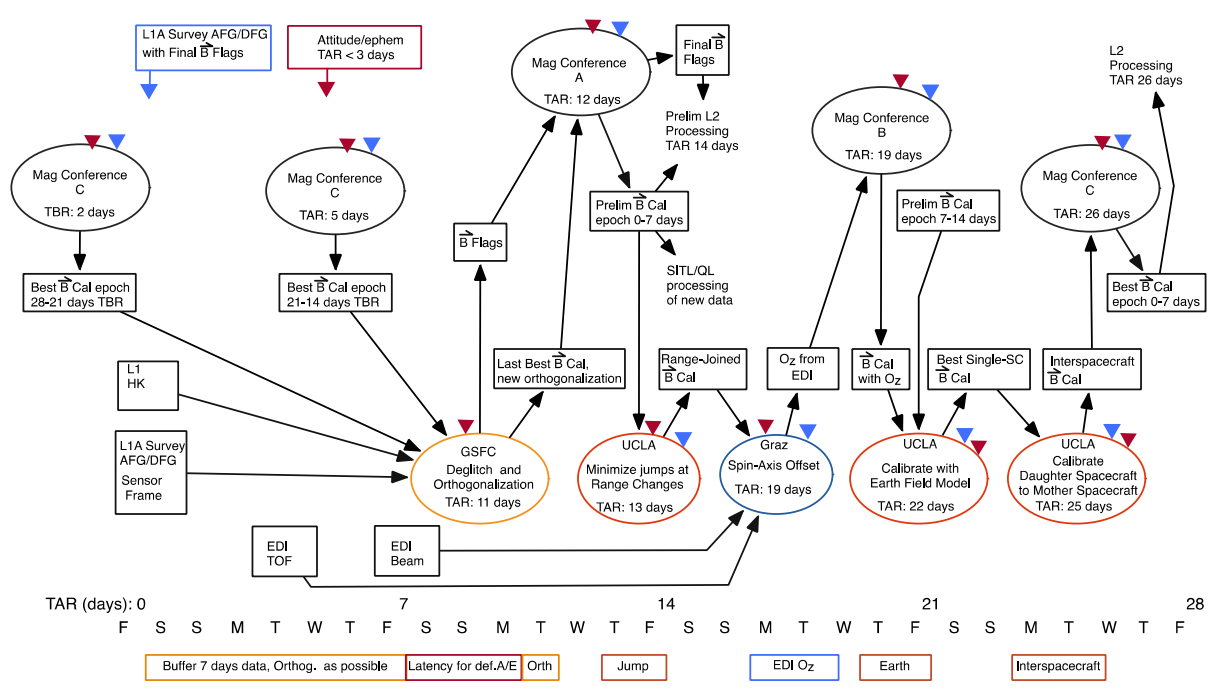

Fig. 55 A possible calibration data flow, including a rough timeline for each calibration step

to corresponding L1A data in the automated Preliminary Level 2 processing. The most recent epoch contained in the 'Prelim B Cal' file will be the calibration that is applied to new L1B and Quicklook data.

5. Determine if it is necessary to upload these parameters for on-board magnetometer calibration in order to maintain proper EDI operation and provide sufficient accuracy for particle pitch angles.

The objectives of Mag Conference ' $\mathrm{B}$ ' would be to discuss the calibrations that have been applied to week of data 19 to 13 days in the past, including range joining and the spin axis offset $\left(O_{z}\right)$ obtained by EDI.

The objectives of Mag Conference ' $\mathrm{C}$ ', held within 26 days after receipt of the data, include:

1. Discuss whether the adjustments to $O_{z}$ necessary to get good inter-spacecraft calibration lie within the error bars of the EDI $O_{z}$ calculation.

2. Discuss any other concerns with the data or calibrations from 26 to 20 days in the past that need to be addressed before the data is released as Level 2.

3. Configuration control and release a 'Best B Cal' file that contains the combined results of all calibration techniques for the week in question. The 'Best B Cal' file then becomes the starting point for the preliminary calibration of newly received data.

Note that the 'Best B Cal' file and all intermediate versions of this file store calibrations in a format that transforms L1A data to the best available calibrated data. Therefore, all calibration and data processing starts with L1A data. GSFC provides software that each institution will incorporate into its calibration software to generate calibrated data, given L1A data, a calibration file and 'B Flag' inputs. This software will be derived from the Preliminary Level 2 software that GSFC delivers to the SDC, which is described in detail in Sect. 6.4.

The initial calibration process performed at GSFC is shown in detail in Fig. 56. The process begins with the Best B Cal file, and outputs the GSFC B Cal file for input to Mag Conference 'A'. The process requires L1A Survey AFG/DFG data, housekeeping data, and 


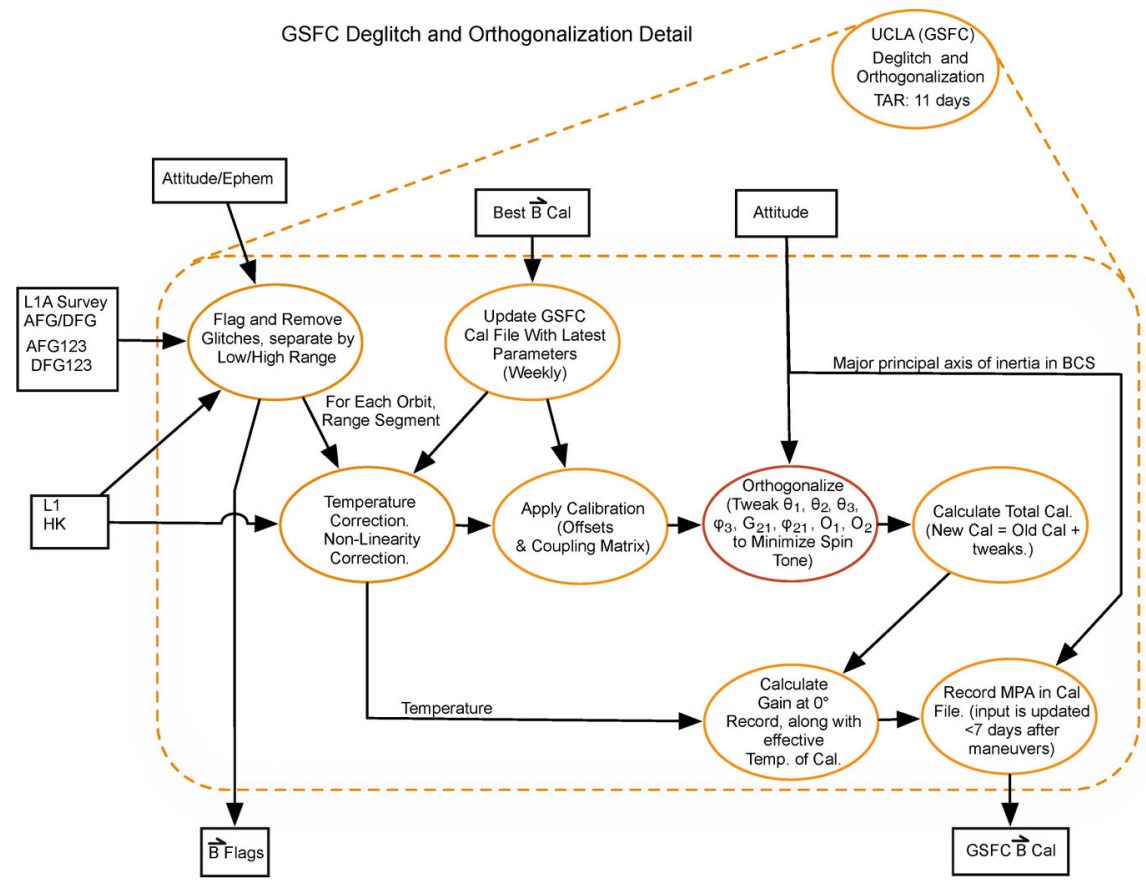

Fig. 56 Data flow for identifying glitches and orthogonalizing the data

definitive attitude and ephemeris data. Note that Attitude/Ephem and L1 HK are shown as inputs to the "Flag and Remove Glitches" process, in order to ensure that all inputs that might possibly help in identifying glitches on orbit will be available to the process. This way, the process may be updated after launch without requiring changes in the interface. Temperature correction (as well as non-linearity correction, for DFG high range only) is applied before attempting to optimize the calibration parameters to minimize spin tone. The temperature correction depends on the sensor temperatures from instrument housekeeping data. Definitive attitude data is used to get precise spin phase for despinning the data. To decouple the gain calibration from the temperature correction, gains are adjusted to an effective temperature of $0{ }^{\circ} \mathrm{C}$. Although it is not required for orthogonalization, this process extracts the MPA from the definitive attitude data and records it in the calibration file, so that the all information required to transform from sensor coordinates to BCS is contained within each record of the calibration file.

\subsection{Level 2 Processing}

Level 2 processing deglitches the data, according to the 'B Flags' file, applies a temperature correction, applies a non-linearity correction for DFG high range only, and then applies the best calibrations, as illustrated in Fig. 57. Finally, it makes coordinate transformations and outputs the data in the desired coordinate systems. The transformations that yield OMB and DMPA coordinates are the same as described in Sect. 6.2. The transformation to GSE coordinates requires definitive attitude and ephemeris, and may use the full star tracker solution, which should resolve the full nutation motion, to remove the nutation signal from the data. 


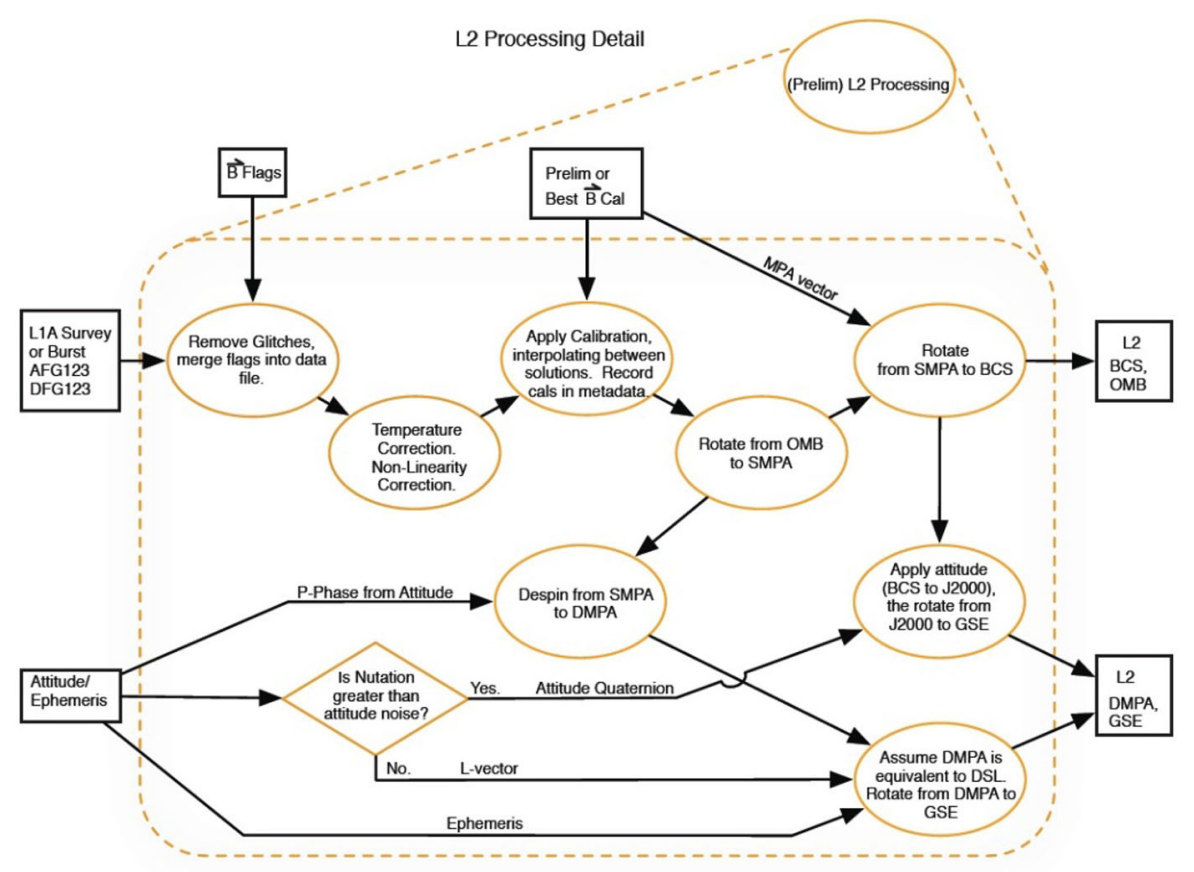

Fig. 57 The same processes are used for L2pre and L2 processing

Once the calibrations have been determined with proper scientific oversight, this L2 process can generate L2pre or L2 data automatically from L1A data (e.g. at the SDC), using the appropriate calibration file and 'B Flags' files as inputs. The same process is used to process burst data, using the calibrations that were calculated from corresponding survey data. This reduces the need for transfer of large data files between GSFC and the SDC, primarily because there is no need to transfer burst data files, which are not required for GSFC calibration activities.

The L2 'Best Science' process will initially be implemented with software that is nearly identical to-but separate from - the L2pre software, allowing for the L2 processing to evolve to allow for higher accuracy without changing with the L2pre processing that is required to work operationally at the SDC with lower data latency and accuracy requirements. At time of writing, it is planned that L2 data production will be performed at UCLA, which will transfer the L2 files to SDC.

\section{Concluding Remarks}

The success of a magnetic field investigation depends on many factors. Redundancy with independent designs is critical to ensuring a single-point failure does not prevent the achievement of the level-one scientific objectives. A magnetically clean spacecraft is needed to ensure that the care that was expended in the design of the instruments results in an accurate measurement of the ambient magnetic field. Constant vigilance on the inter-calibration of the spacecraft allows the accurate intercomparison of the data from the four spacecraft which is needed to calculate the currents flowing in the plasma. Rapid data processing is needed 
to optimize the return of the key high-resolution data stored on the spacecraft. Finally, the sharing of data among all the teams and working together across instrument boundaries is needed to understand the meaning of the measurements and to translate them to theoretical understanding.

Acknowledgements The MMS magnetometer team is very grateful for the support and assistance provided to us by many individuals in the construction, test, and calibration of the magnetometers. At UCLA, the sensors were carefully wound by William Greer. Assistance with calibration at IWF was provided by S. Leitner, and at TU-BS., K.H. Fornacon, with the guidance of K.-H. Glassmeier. Assisting with magnetic cleanliness were S. Liang at APL, G. Won of GSFC, and J. Stone. We also wish to thank the project staff at UNH and SWRI, without whose support this effort would not have been possible.

Open Access This article is distributed under the terms of the Creative Commons Attribution License which permits any use, distribution, and reproduction in any medium, provided the original author(s) and the source are credited.

\section{References}

R.S. Caprari, Meas. Sci. Technol. 6, 593-597 (1995)

J.W. Dungey, Phys. Rev. Lett. 6, 47-48 (1961)

J.W. Dungey, in Geophysics: The Earth's Environment, ed. by C. Dewitt, J. Hieblot, A. Lebeau (Gordon and Breach, New York, 1963), pp. 505-550

K.-H. Glassmeier, I. Richter, A. Diedrich, G. Mussmann, U. Auster, U. Motschmann, A. Balogh, C. Carr, E. Cupido, A. Coates, M. Rother, K. Schwingenschuh, K. Szegö, B. Tsurutani, Space Sci. Rev. (2007). doi:10.1007/s11214-006-9114-x

E. Georgescu, H. Vaith, K.-H. Fornacon, U. Auster, A. Balogh, C. Carr, M. Chutter, M. Dunlop, M. Foerster, K.-H. Glassmeier, J. Gloag, G. Paschmann, J. Quinn, R. Torbert, in Cluster and Double Star Symposium. ESA Special Publ., vol. 598 (2006)

E.L. Kepko, K.K. Khurana, M.G. Kivelson, IEEE Trans. Magn. 32, 377 (1996)

K.K. Khurana, E.L. Kepko, M.G. Kivelson, R.C. Elphic, IEEE Trans. Magn. 32, 5193 (1996)

H.K. Leinweber, In-flight calibration of space-borne magnetometers. Ph.D. Thesis, Graz University of Technology (2012)

H.K. Leinweber, C.T. Russell, K. Torkar, T.L. Zhang, V. Angelopoulos, Meas. Sci. Technol. 19, 5 (2008)

H.K. Leinweber, C.T. Russell, K. Torkar, Meas. Sci. Technol. (2012). doi:10.1088/0957-0233/23/10/105003

H.K. Leinweber, C.T. Russell, K. Torkar, IEEE Trans. Magn. 49, 5264-5269 (2013)

W. Magnes, M. Oberst, A. Valavanoglou, H. Hauer, C. Hagen, I. Jernej, H. Neubauer, W. Baumjohann, D. Pierce, J. Means, P. Falkner, Meas. Sci. Technol. (2008). doi:10.1088/09570233/19/11/115801

R.L. McPherron, C.T. Russell, M.P. Aubry, J. Geophys. Res. 78, 3131-3149 (1973)

R.F. Nakamura, W. Baumjohann, C. Moulkis, L.M. Kistler, A. Runov, M. Volwerk, Y. Asano, Z. Voros, T.L. Zhang, B. Klecker, H. Reme, A. Balogh, Geophys. Res. Lett. (2004). doi:10.1029/2004GL019558

R.F. Nakamura, R. Plaschke, R. Tuebenbacher, L. Giner, W. Baumjohann, W. Magnes, M. Steller, R.B. Torbert, H. Vaith, M. Chutter, K.-H. Fornacon, K.-H. Glassmeier, C. Carr, Geosci. Instrum. Method. Data Syst. Discuss. (2014). doi:10.5194/gi-3-1-2014

N.F. Ness, C.S. Scearce, J.B. Seek, J. Geophys. Res. 69, 3531 (1964)

E.N. Parker, Astrophys. J. Suppl. Ser. 77(8), 177 (1963)

G. Paschmann, B.U.Ö. Sonnerup, I. Papamastorakis, N. Sckopke, G. Haerendel, S.J. Bame, J.R. Asbridge, J.T. Gosling, C.T. Russell, R.C. Elphic, Nature 282, 243-246 (1979)

H.E. Petschek, in Magnetic Field Annihilation in AAS-NASA Symposium on the Physics of Solar Flares. NASA SP-50, ed. by W.N. Hess (1964), pp. 425-439

F. Plaschke, R. Nakamura, H.K. Leinweber, M. Chutter, H. Vaith, W. Baumjohann, M. Steller, W. Magnes, Meas. Sci. Technol. (2014, accepted)

C.T. Russell, R.L. McPherron, Space Sci. Rev. 15, 205-266 (1973)

P.A. Sweet, Suppl. Nuovo Cim. 8(10), 188 (1958)

R.B. Torbert, C.T. Russell, W. Magnes, R.E. Ergun, P.A. Lindquist, O. LeContel, H. Vaith, J. Macri, S. Myers, D. Rau, J. Needell, B. King, M. Granoff, M. Chutter, I. Dors, G. Olsson, Y. Khotyaintser, A. Erikson, G.A. Kletzing, S. Bounds, B.J. Anderson, W. Baumjohann, M. Stellar, K. Bromund, G. Le, R. Nakamura, R.J. Strangeway, S. Tucker, J. Westfall, D. Fisher, F. Plaschke, Space Sci. Rev. (2015, this issue) 